\title{
GALOIS REPRESENTATIONS MODULO $p$ AND COHOMOLOGY OF HILBERT MODULAR VARIETIES
}

\author{
By MLADEN DIMITROV
}

ABSTRACT. - The aim of this paper is to extend some arithmetic results on elliptic modular forms to the case of Hilbert modular forms. Among these results let us mention:

- control of the image of Galois representations modulo $p$,

- Hida's congruence criterion outside an explicit set of primes,

- freeness of the integral cohomology of a Hilbert modular variety over certain local components of the Hecke algebra and Gorenstein property of these local algebras.

We study the arithmetic properties of Hilbert modular forms by studying their modulo $p$ Galois representations and our main tool is the action of inertia groups at primes above $p$. In order to determine this action, we compute the Hodge-Tate (resp. Fontaine-Laffaille) weights of the $p$-adic (resp. modulo $p$ ) étale cohomology of the Hilbert modular variety. The cohomological part of our paper is inspired by the work of Mokrane, Polo and Tilouine on the cohomology of Siegel modular varieties and builds upon geometric constructions of Tilouine and the author.

(c) 2005 Elsevier SAS

RÉSUMÉ. - Le but de cet article est de généraliser certains résultats arithmétiques sur les formes modulaires elliptiques au cas des formes modulaires de Hilbert. Parmi ces résultats citons :

- détermination de l'image de représentations galoisiennes modulo $p$,

- critère de congruence de Hida en dehors d'un ensemble explicite de premiers,

- liberté de la cohomologie entière de la variété modulaire de Hilbert sur certaines composantes locales de l'algèbre de Hecke et la propriété de Gorenstein de celles-ci.

L'étude des propriétés arithmétiques des formes modulaires de Hilbert se fait à travers leurs représentations galoisiennes modulo $p$ et l'outil principal est l'action des groupes d'inertie aux premiers au-dessus de $p$. Cette action est déterminée par le calcul des poids de Hodge-Tate (resp. Fontaine-Laffaille) de la cohomologie étale $p$-adique (resp. modulo $p$ ) de la variété modulaire de Hilbert. La partie cohomologique de cet article est inspirée par le travail de Mokrane, Polo et Tilouine sur la cohomologie des variétés modulaires de Siegel et repose sur des constructions géométriques de Tilouine et l'auteur.

(C) 2005 Elsevier SAS

\section{Contents}

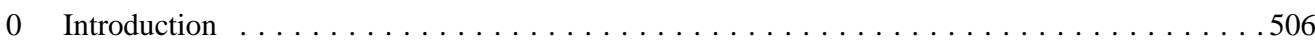

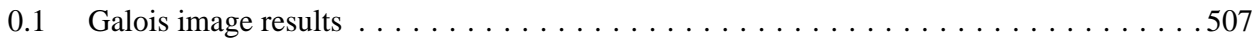

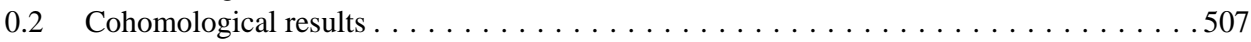

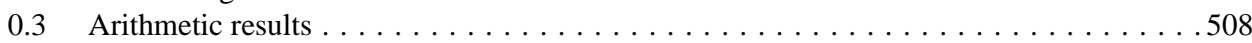

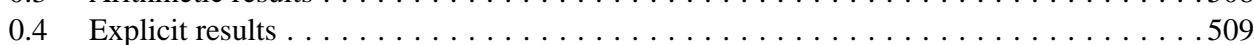

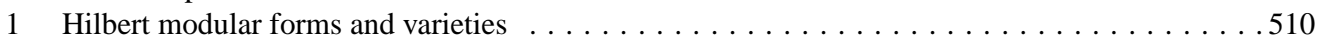

1.1 Analytic Hilbert modular varieties . . . . . . . . . . . . . . . . . . . . . 510

ANNALES SCIENTIFIQUES DE L'ÉCOLE NORMALE SUPÉRIEURE

0012-9593/04/@ 2005 Elsevier SAS. All rights reserved 
1.2 Analytic Hilbert modular forms $\ldots \ldots \ldots \ldots \ldots \ldots \ldots \ldots \ldots \ldots$

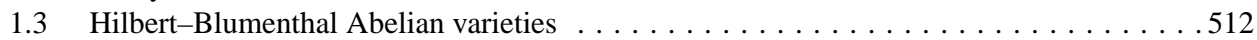

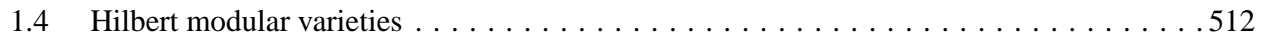

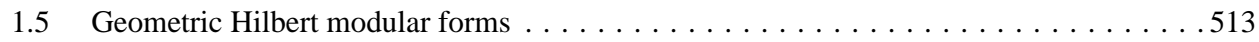

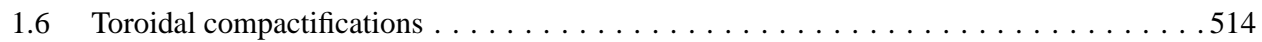

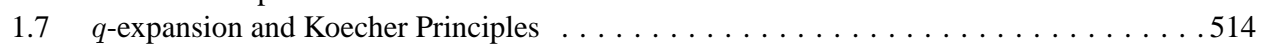

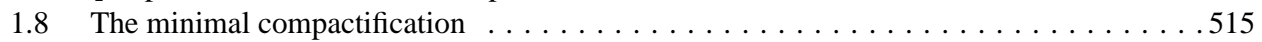

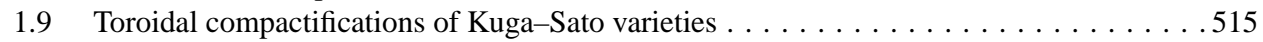

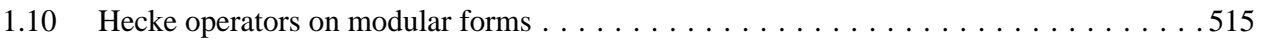

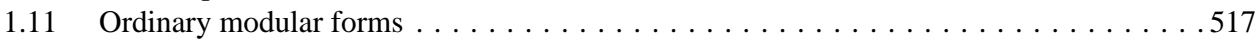

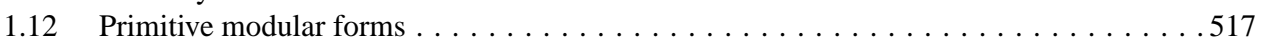

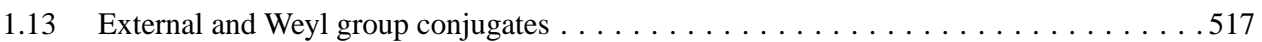

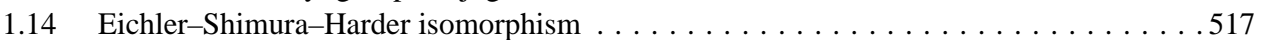

2 Hodge-Tate weights of Hilbert modular varieties . . . . . . . . . . . . . . . . . . . 519

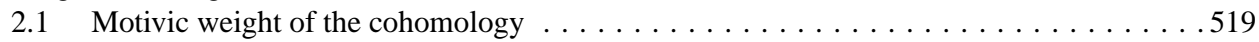

2.2 The Bernstein-Gelfand-Gelfand complex over $\overline{\mathbb{Q}} \ldots \ldots \ldots \ldots \ldots \ldots$

2.3 Hodge-Tate decomposition of $\mathrm{H}^{\bullet}\left(M \otimes \overline{\mathbb{Q}}_{p}, \mathbb{V}_{n}\left(\overline{\mathbb{Q}}_{p}\right)\right) \ldots \ldots \ldots \ldots \ldots \ldots$

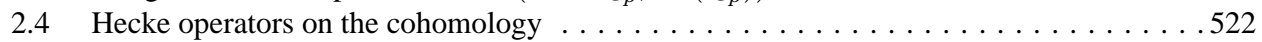

2.5 Hodge-Tate weights of $\otimes \operatorname{Ind}_{F}^{\mathbb{Q}} \rho$ in the crystalline case $\ldots \ldots \ldots \ldots \ldots \ldots \ldots \ldots$

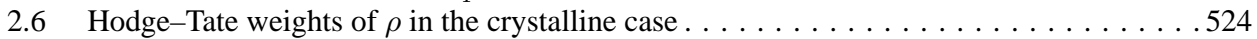

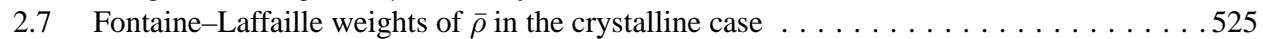

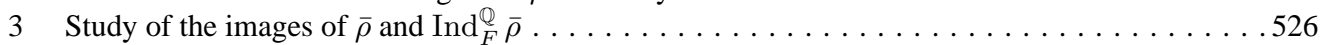

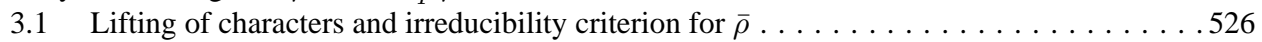

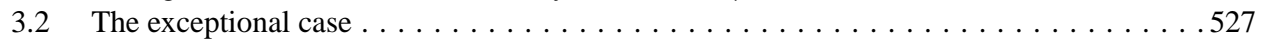

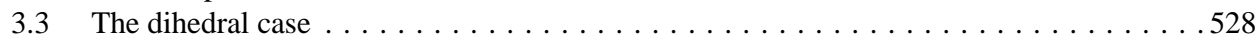

3.4 The image of $\bar{\rho}$ is "large" . . . . . . . . . . . . . . . . . . . . . . . . . . . . .529

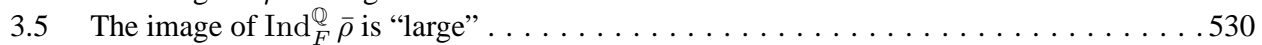

4 Boundary cohomology and congruence criterion $\ldots \ldots \ldots \ldots \ldots \ldots \ldots \ldots \ldots \ldots$

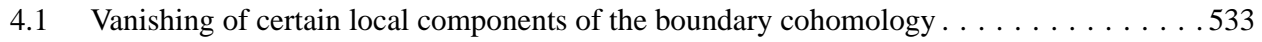

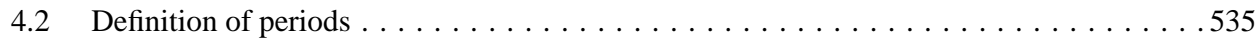

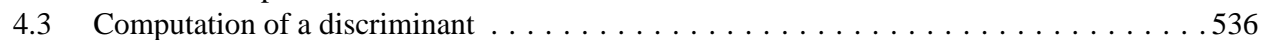

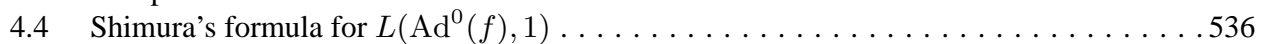

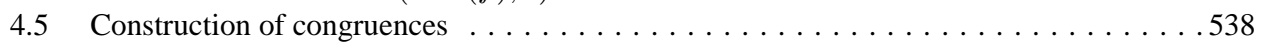

5 Fontaine-Laffaille weights of Hilbert modular varieties . . . . . . . . . . . . . . . . . 539

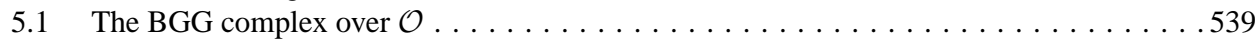

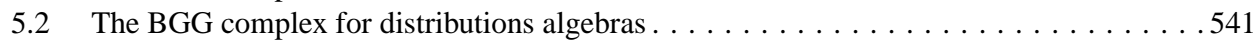

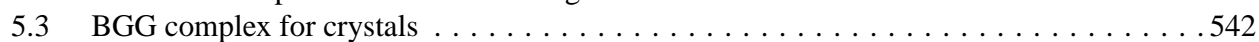

6 Integral cohomology over certain local components of the Hecke algebra . . . . . . . . . . . 544

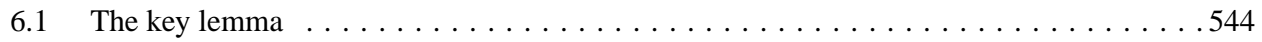

6.2 Localized cohomology of the Hilbert modular variety . . . . . . . . . . . . . . 545

6.3 On the Gorenstein property of the Hecke algebra . . . . . . . . . . . . . . . . . . . 546

6.4 An application to $p$-adic ordinary families $\ldots \ldots \ldots \ldots \ldots \ldots \ldots \ldots \ldots \ldots \ldots \ldots$

List of symbols . . . . . . . . . . . . . . . . . . . . . . . . . . . . . . . . . . 549

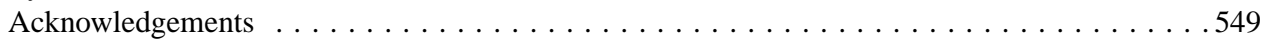

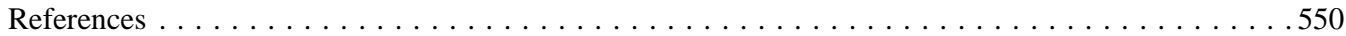

\section{Introduction}

Let $F$ be a totally real number field of degree $d$, ring of integers $\mathfrak{o}$ and different $\mathfrak{d}$. Denote by $\widetilde{F}$ the Galois closure of $F$ in $\overline{\mathbb{Q}}$ and by $J_{F}$ the set of all embeddings of $F$ into $\overline{\mathbb{Q}} \subset \mathbb{C}$.

We fix an ideal $\mathfrak{n} \subset \mathfrak{o}$ and we put $\Delta=\mathrm{N}_{F / \mathbb{Q}}(\mathfrak{n} \mathfrak{d})$.

$4^{\mathrm{e}}$ SÉRIE - TOME $38-2005-\mathrm{N}^{\circ} 4$ 
For a weight $k=\sum_{\tau \in J_{F}} k_{\tau} \tau \in \mathbb{Z}\left[J_{F}\right]$ as in Definition 1.1 we put $k_{0}=\max \left\{k_{\tau} \mid \tau \in J_{F}\right\}$. If $\psi$ is a Hecke character of $F$ of conductor dividing $\mathfrak{n}$ and type $2-k_{0}$ at infinity, we denote by $S_{k}(\mathfrak{n}, \psi)$ the corresponding space of Hilbert modular cuspforms (see Definition 1.3).

Let $f \in S_{k}(\mathfrak{n}, \psi)$ be a newform, that is, a primitive normalized eigenform. For all ideals $\mathfrak{a} \subset \mathfrak{o}$, we denote by $c(f, \mathfrak{a})$ the eigenvalue of the standard Hecke operator $T_{\mathfrak{a}}$ on $f$.

Let $p$ be a prime number and let $\iota_{p}: \overline{\mathbb{Q}} \hookrightarrow \overline{\mathbb{Q}}_{p}$ be an embedding.

Denote by $E$ a sufficiently large $p$-adic field with ring of integers $\mathcal{O}$, maximal ideal $\mathcal{P}$ and residue field $\kappa$.

\subsection{Galois image results}

The absolute Galois group of a field $L$ is denoted by $\mathcal{G}_{L}$. By results of Taylor [40,41] and Blasius and Rogawski [1] there exists a continuous representation $\rho=\rho_{f, p}: \mathcal{G}_{F} \rightarrow \mathrm{GL}_{2}(E)$ which is absolutely irreducible, totally odd, unramified outside $\mathfrak{n} p$ and such that for each prime ideal $v$ of $\mathfrak{o}$, not dividing $p \mathfrak{n}$, we have:

$$
\operatorname{tr}\left(\rho\left(\operatorname{Frob}_{v}\right)\right)=\iota_{p}(c(f, v)), \quad \operatorname{det}\left(\rho\left(\operatorname{Frob}_{v}\right)\right)=\iota_{p}(\psi(v)) \mathrm{N}_{F / \mathbb{Q}}(v),
$$

where $\operatorname{Frob}_{v}$ denotes a geometric Frobenius at $v$.

By taking a Galois stable $\mathcal{O}$-lattice, we define $\bar{\rho}=\rho_{f, p} \bmod \mathcal{P}: \mathcal{G}_{F} \rightarrow \mathrm{GL}_{2}(\kappa)$, whose semisimplification is independent of the particular choice of a lattice.

The following proposition is a generalization to the Hilbert modular case of results of Serre [37] and Ribet [35] on elliptic modular forms (see Propositions 3.1, 3.8 and 3.17).

Proposition 0.1. - (i) For all but finitely many primes $p$,

$\left(\mathbf{I r r}_{\bar{\rho}}\right) \bar{\rho}$ is absolutely irreducible.

(ii) If $f$ is not a theta series, then for all but finitely many primes $p$,

$\left(\mathbf{L I} \mathbf{I}_{\bar{\rho}}\right)$ there exists a power $q$ of $p$ such that $\mathrm{SL}_{2}\left(\mathbb{F}_{q}\right) \subset \operatorname{im}(\bar{\rho}) \subset \kappa^{\times} \mathrm{GL}_{2}\left(\mathbb{F}_{q}\right)$.

(iii) Assume that $f$ is not a twist by a character of any of its internal conjugates and is not a theta series. Then for all but finitely many primes $p$,

$\left(\mathbf{L} \mathbf{I}_{\text {Ind } \bar{\rho}}\right)$ there exist a power $q$ of $p$, a partition $J_{F}=\coprod_{i \in I} J_{F}^{i}$ and for all $\tau \in J_{F}^{i}$ an element $\sigma_{i, \tau} \in \operatorname{Gal}\left(\mathbb{F}_{q} / \mathbb{F}_{p}\right)$ such that $\left(\tau \neq \tau^{\prime} \Longrightarrow \sigma_{i, \tau} \neq \sigma_{i, \tau^{\prime}}\right)$ and $\operatorname{Ind}_{F}^{\mathbb{Q}} \bar{\rho}: \mathcal{G}_{\widehat{F}^{\prime \prime}} \rightarrow \mathrm{SL}_{2}\left(\mathbb{F}_{q}\right)^{J_{F}}$ factors as a surjection $\mathcal{G}_{\widehat{F}^{\prime \prime}} \rightarrow \mathrm{SL}_{2}\left(\mathbb{F}_{q}\right)^{I}$ followed by the map $\left(M_{i}\right)_{i \in I} \mapsto\left(M_{i}^{\sigma_{i, \tau}}\right)_{i \in I, \tau \in J_{F}^{i}}$, where $\widehat{F}^{\prime \prime}$ denotes the compositum of $\widetilde{F}$ and the fixed field of $\left(\operatorname{Ind}_{F}^{\mathbb{Q}} \bar{\rho}\right)^{-1}\left(\mathrm{SL}_{2}\left(\mathbb{F}_{q}\right)^{J_{F}}\right)$.

\subsection{Cohomological results}

Let $Y_{/ \mathbb{Z}\left[\frac{1}{\Delta}\right]}$ be the Hilbert modular variety of level $K_{1}(\mathfrak{n})$ (see Section 1.4). Consider the $p$-adic étale cohomology $\mathrm{H}^{\bullet}\left(Y_{\overline{\mathbb{Q}}}, \mathbb{V}_{n}\left(\overline{\mathbb{Q}}_{p}\right)\right)$, where $\mathbb{V}_{n}\left(\overline{\mathbb{Q}}_{p}\right)$ denotes the local system of weight $n=\sum_{\tau \in J_{F}}\left(k_{\tau}-2\right) \tau \in \mathbb{N}\left[J_{F}\right]$ (see Section 2.1). By a result of Brylinski and Labesse [3] the subspace $W_{f}:=\bigcap_{\mathfrak{a} \subset \mathfrak{o}} \operatorname{ker}\left(T_{\mathfrak{a}}-c(f, \mathfrak{a})\right)$ of $\mathrm{H}^{d}\left(Y_{\overline{\mathbb{Q}}}, \mathbb{V}_{n}\left(\overline{\mathbb{Q}}_{p}\right)\right)$ is isomorphic, as $\mathcal{G}_{\widetilde{F}}$-module and after semi-simplification, to the tensor induced representation $\otimes \operatorname{Ind}_{F}^{\mathbb{Q}} \rho$.

Assume that

(I) $p$ does not divide $\Delta$.

Then $Y$ has smooth toroidal compactifications over $\mathbb{Z}_{p}$ (see [10]). For each $J \subset J_{F}$, we put $|p(J)|=\sum_{\tau \in J}\left(k_{0}-m_{\tau}-1\right)+\sum_{\tau \in J_{F} \backslash J} m_{\tau}$, where $m_{\tau}=\left(k_{0}-k_{\tau}\right) / 2 \in \mathbb{N}$. By applying a method of Chai and Faltings [15, Chapter VI] one can prove (see [11, Theorem 7.8, Corollary 7.9]). 
THEOREM 0.2. - Assume that $p$ does not divide $\Delta$. Then

(i) the Galois representation $\mathrm{H}^{j}\left(Y_{\overline{\mathbb{Q}}}, \mathbb{V}_{n}\left(\overline{\mathbb{Q}}_{p}\right)\right)$ is crystalline at $p$ and its Hodge-Tate weights belong to the set $\left\{|p(J)|, J \subset J_{F},|J| \leqslant j\right\}$, and

(ii) the Hodge-Tate weights of $W_{f}$ are given by the multiset $\left\{|p(J)|, J \subset J_{F}\right\}$.

For our main arithmetic applications we need to establish a modulo $p$ version of the above theorem. This is achieved under the following additional assumption:

(II) $p-1>\sum_{\tau \in J_{F}}\left(k_{\tau}-1\right)$.

The integer $\sum_{\tau \in J_{F}}\left(k_{\tau}-1\right)$ is equal to the difference $\left|p\left(J_{F}\right)\right|-|p(\emptyset)|$ between the largest and smallest Hodge-Tate weights of the cohomology of the Hilbert modular variety. We use (I) and (II) in order to apply Fontaine-Laffaille's Theory [17] as well as Faltings' Comparison Theorem modulo $p$ [14]. By adapting to the case of Hilbert modular varieties some techniques developed by Mokrane, Polo and Tilouine [31,33] for Siegel modular varieties, such as the construction of an integral Bernstein-Gelfand-Gelfand complex for distribution algebras, we compute the Fontaine-Laffaille weights of $\mathrm{H}^{\bullet}\left(Y_{\overline{\mathbb{Q}}}, \mathbb{V}_{n}(\kappa)\right)$ (see Theorem 5.13).

\subsection{Arithmetic results}

Consider the $\mathcal{O}$-module of interior cohomology $\mathrm{H}_{!}^{d}\left(Y, \mathbb{V}_{n}(\mathcal{O})\right)^{\prime}$, defined as the image of $\mathrm{H}_{c}^{d}\left(Y, \mathbb{V}_{n}(\mathcal{O})\right)$ in $\mathrm{H}^{d}\left(Y, \mathbb{V}_{n}(E)\right)$. Let $\mathbb{T}=\mathcal{O}\left[T_{\mathfrak{a}}, \mathfrak{a} \subset \mathfrak{o}\right]$ be the full Hecke algebra acting on it, and let $\mathbb{T}^{\prime} \subset \mathbb{T}$ be the subalgebra generated by the Hecke operators outside a finite set of places containing those dividing $\mathfrak{n} p$. Denote by $\mathfrak{m}$ the maximal ideal of $\mathbb{T}$ corresponding to $f$ and $\iota_{p}$ and put $\mathfrak{m}^{\prime}=\mathfrak{m} \cap \mathbb{T}^{\prime}$.

THEOREM 0.3. - Assume that the conditions (I) and (II) from Section 0.2 hold.

(i) If $\left(\operatorname{Irr}_{\bar{\rho}}\right)$ holds, $d(p-1)>5 \sum_{\tau \in J_{F}}\left(k_{\tau}-1\right)$ and

(MW) the middle weight $\frac{\left|p\left(J_{F}\right)\right|+|p(\emptyset)|}{2}=\frac{d\left(k_{0}-1\right)}{2}$ does not belong to $\left\{|p(J)|, J \subset J_{F}\right\}$, then the local component $\mathrm{H}_{\partial}^{\bullet}\left(Y, \mathbb{V}_{n}(\mathcal{O})\right)_{\mathfrak{m}^{\prime}}$ of the boundary cohomology vanishes, and the Poincaré pairing $\mathrm{H}_{!}^{d}\left(Y, \mathbb{V}_{n}(\mathcal{O})\right)_{\mathfrak{m}^{\prime}}^{\prime} \times \mathrm{H}_{!}^{d}\left(Y, \mathbb{V}_{n}(\mathcal{O})\right)_{\mathfrak{m}^{\prime}}^{\prime} \rightarrow \mathcal{O}$ is a perfect duality.

(ii) If $\left(\mathbf{L} \mathbf{I}_{\text {Ind } \bar{\rho}}\right)$ holds, then $\mathrm{H}^{\bullet}\left(Y, \mathbb{V}_{n}(\mathcal{O})\right)_{\mathfrak{m}^{\prime}}=\mathrm{H}^{d}\left(Y, \mathbb{V}_{n}(\mathcal{O})\right)_{\mathfrak{m}^{\prime}}$ is a free $\mathcal{O}$-module of finite rank and its Pontryagin dual is isomorphic to $\mathrm{H}^{d}\left(Y, \mathbb{V}_{n}(E / \mathcal{O})\right)_{\mathfrak{m}^{\prime}}$.

The proof involves a "local-global" Galois argument. The first part is proved in Theorem 4.4 using Lemma 4.2(ii) and a theorem of Pink [32] on the étale cohomology of a local system restricted to the boundary of $Y$. The second part is proved in Theorem 6.6 using Lemma 6.5 and the computation of the Fontaine-Laffaille weights of the cohomology from Theorem 5.13. The technical assumptions are needed in the lemmas. Since the conclusion of Lemma 6.5 is stronger then the one of Lemma 4.2(ii) we see that the results of Theorems 0.3(i) and A (see below) remain true under the assumptions $(\mathbf{I}),(\mathbf{I I})$ and $\left(\mathbf{L I}_{\text {Ind } \bar{\rho}}\right)$.

Let $L^{*}\left(\operatorname{Ad}^{0}(f), s\right)$ be the imprimitive adjoint $L$-function of $f$ and let $\Gamma\left(\operatorname{Ad}^{0}(f), s\right)$ be the corresponding Euler factor (see Section 4.4). We denote by $\Omega_{f} \in \mathbb{C}^{\times} / \mathcal{O}^{\times}$any two complementary periods defined by the Eichler-Shimura-Harder isomorphism (see Section 4.2).

THEOREM A (Theorem 4.11). - Let $f$ and $p$ be such that (I), $\left(\operatorname{Irr}_{\bar{\rho}}\right)$ and (MW) hold, and $p-1>\max \left(1, \frac{5}{d}\right) \sum_{\tau \in J_{F}}\left(k_{\tau}-1\right)$. Assume that $\iota_{p}\left(\frac{\Gamma\left(\operatorname{Ad}^{0}(f), 1\right) L^{*}\left(\operatorname{Ad}^{0}(f), 1\right)}{\Omega_{f}^{+} \Omega_{f}^{-}}\right) \in \mathcal{P}$. Then there exists another normalized eigenform $g \in S_{k}(\mathfrak{n}, \psi)$ such that $f \equiv g(\bmod \mathcal{P})$, in the sense that $c(f, \mathfrak{a}) \equiv c(g, \mathfrak{a})(\bmod \mathcal{P})$ for each ideal $\mathfrak{a} \subset \mathfrak{o}$.

The proof follows closely the original one given by Hida [21] in the elliptic modular case, and uses Theorem 0.3(i) as well as a formula of Shimura relating $L^{*}\left(\operatorname{Ad}^{0}(f), 1\right)$ to the Petersson 
inner product of $f$ (see (19)). Let us note that Ghate [18] has obtained a very similar result when the weight $k$ is parallel. A converse for Theorem A is provided by the second part of the following

THEOREM B (Theorem 6.7). - Let $f$ and $p$ be such that $(\mathbf{I}),(\mathbf{I I})$ and $\left(\mathbf{L I}_{\text {Ind } \bar{\rho}) \text { hold. Then }}\right.$

(i) $\mathrm{H}^{\bullet}\left(Y, \mathbb{V}_{n}(\kappa)\right)[\mathfrak{m}]=\mathrm{H}^{d}\left(Y, \mathbb{V}_{n}(\kappa)\right)[\mathfrak{m}]$ is a $\kappa$-vector space of dimension $2^{d}$.

(ii) $\mathrm{H}^{\bullet}\left(Y, \mathbb{V}_{n}(\mathcal{O})\right)_{\mathfrak{m}}=\mathrm{H}^{d}\left(Y, \mathbb{V}_{n}(\mathcal{O})\right)_{\mathfrak{m}}$ is free of rank $2^{d}$ over $\mathbb{T}_{\mathfrak{m}}$.

(iii) $\mathbb{T}_{\mathfrak{m}}$ is Gorenstein.

By [30] it is enough to prove (i), which is a consequence of Theorem 0.3(ii) and the $q$ expansion principle Section 1.7. This theorem is due, under milder assumptions, to Mazur [30] for $F=\mathbb{Q}$ and $k=2$, and to Faltings and Jordan [16] for $F=\mathbb{Q}$. The Gorenstein property is proved by Diamond [8] when $F$ is quadratic and $k=(2,2)$ under the assumptions (I), (II) and $\left(\operatorname{Irr}_{\bar{\rho}}\right)$. We expect that Diamond's approach via intersection cohomology could be generalized in order to prove the Gorenstein property of $\mathbb{T}_{\mathfrak{m}}$ under the assumptions (I), (II) and $\left(\mathbf{L} \mathbf{I}_{\bar{\rho}}\right)$ (see Lemma 4.2(i) and Remark 4.3).

When $f$ is ordinary at $p$ (see Definition 1.13) we can replace the assumptions (I) and (II) of Theorems A and B by the weaker assumptions that $p$ does not divide $\mathrm{N}_{F / \mathbb{Q}}(\mathfrak{d})$ and that $k(\bmod p-1)$ satisfies (II) (see Corollary 6.10$)$. The proof uses Hida's families of $p$-adic ordinary Hilbert modular forms. We prove an exact control theorem for the ordinary part of the cohomology of the Hilbert modular variety, and give a new proof of Hida's exact control theorem for the ordinary Hecke algebra (see Proposition 6.9).

Theorems A and B prove that the congruence ideal associated to the $\mathcal{O}$-algebra homomorphism $\mathbb{T} \rightarrow \mathcal{O}, T_{\mathfrak{a}} \mapsto \iota_{p}(c(f, \mathfrak{a}))$ is generated by $\iota_{p}\left(\frac{\Gamma\left(\operatorname{Ad}^{0}(f), 1\right) L^{*}\left(\operatorname{Ad}^{0}(f), 1\right)}{\Omega_{f}^{+} \Omega_{f}^{-}}\right)$. In a subsequent paper [12] we relate it to the fitting ideal of the Bloch-Kato Selmer group associated to $\operatorname{Ad}^{0}(\rho) \otimes E / \mathcal{O}$. An interesting question is whether $\Omega_{f}$ are the periods involved in the Bloch-Kato conjecture for the motive $\operatorname{Ad}^{0}(f)$ constructed by Blasius and Rogawski [1] (see the work of Diamond, Flach and Guo [9] for the elliptic modular case).

\subsection{Explicit results}

By a classical theorem of Dickson, if $\left(\operatorname{Irr}_{\bar{\rho}}\right)$ holds but $\left(\mathbf{L I}_{\bar{\rho}}\right)$ fails, then the image of $\bar{\rho}$ in $\mathrm{PGL}_{2}(\kappa)$ should be isomorphic to a dihedral, tetrahedral, octahedral or icosahedral group. Using this fact as well as Proposition 3.1, Section 3.2, Propositions 3.5 and 3.13 we obtain the following corollary to Theorems A and B.

Denote by $\mathfrak{o}_{+}^{\times}$(respectively $\mathfrak{o}_{\mathfrak{n}, 1}^{\times}$) the group of totally positive (respectively congruent to 1 modulo $\mathfrak{n}$ ) units of $\mathfrak{o}$.

COROLLARY 0.4. - Let $\epsilon$ be any element of $\mathfrak{o}_{+}^{\times} \cap \mathfrak{o}_{\mathfrak{n}, 1}^{\times}$.

(i) Assume $d=2$ and $k=\left(k_{0}, k_{0}-2 m_{1}\right)$, with $m_{1} \neq 0$. If

$$
p \nmid \Delta \mathrm{N}_{F / \mathbb{Q}}\left(\left(\epsilon^{m_{1}}-1\right)\left(\epsilon^{k_{0}-m_{1}-1}-1\right)\right)
$$

and $p-1>4\left(k_{0}-m_{1}-1\right)$ then Theorem A holds. If additionally the image of $\bar{\rho}$ in $\mathrm{PGL}_{2}(\kappa)$ is not a dihedral group then Theorem $\mathrm{B}$ also holds.

(ii) Assume $d=3$, id $\neq \tau \in J_{F}$ and $k=\left(k_{0}, k_{0}-2 m_{1}, k_{0}-2 m_{2}\right)$, with $0<m_{1}+m_{2} \neq$ $\frac{k_{0}-1}{2}$. If

$$
\begin{aligned}
p \nmid \Delta & \mathrm{N}_{\widetilde{F} / \mathbb{Q}}\left(\left(\tau(\epsilon)^{m_{1}}-\epsilon^{-m_{2}}\right)\left(\tau(\epsilon)^{m_{1}}-\epsilon^{m_{2}+1-k_{0}}\right)\left(\tau(\epsilon)^{m_{1}+1-k_{0}}-\epsilon^{m_{2}}\right)\right. \\
& \left.\times\left(\tau(\epsilon)^{k_{0}-m_{1}-1}-\epsilon^{m_{2}+1-k_{0}}\right)\right)
\end{aligned}
$$


and $p-1>\frac{5}{3}\left(3 k_{0}-2 m_{1}-2 m_{2}-3\right)$ then Theorem A holds. If additionally the image of $\bar{\rho}$ in $\mathrm{PGL}_{2}(\kappa)$ is not a dihedral group then Theorem $\mathrm{B}$ also holds.

\section{Hilbert modular forms and varieties}

We define the algebraic groups $D_{/ \mathbb{Q}}=\operatorname{Res}_{\mathbb{Q}}^{F} \mathbb{G}_{m}, G / \mathbb{Q}=\operatorname{Res}_{\mathbb{Q}}^{F} \mathrm{GL}_{2}$ and $G_{/ \mathbb{Q}}^{*}=G \times_{D} \mathbb{G}_{m}$, where the fiber product is relative to the reduced norm map $\nu: G \rightarrow D$. The standard Borel subgroup of $G$, its unipotent radical and its standard maximal torus are denoted by $B, U$ and $T$, respectively. We identify $D \times D$ with $T$, by $(u, \epsilon) \mapsto\left(\begin{array}{cc}u \epsilon & 0 \\ 0 & u^{-1}\end{array}\right)$.

\subsection{Analytic Hilbert modular varieties}

Let $D(\mathbb{R})_{+}$(respectively $G(\mathbb{R})_{+}$) be the identity component of $D(\mathbb{R})=(F \otimes \mathbb{R})^{\times}$(respectively of $G(\mathbb{R})$ ). The group $G(\mathbb{R})_{+}$acts by linear fractional transformations on the space $\mathfrak{H}_{F}=\left\{z \in F \otimes \mathbb{C} \mid \operatorname{im}(z) \in D(\mathbb{R})_{+}\right\}$. We have $\mathfrak{H}_{F} \cong \mathfrak{H}^{J_{F}}$, where $\mathfrak{H}=\{z \in \mathbb{C} \mid \operatorname{im}(z)>0\}$ is the Poincaré's upper half-plane (the isomorphism being given by $\xi \otimes z \mapsto(\tau(\xi) z)_{\tau \in J_{F}}$, for $\xi \in F, z \in \mathbb{C}$ ). We consider the unique group action of $G(\mathbb{R})$ on the space $\mathfrak{H}_{F}$ extending the action of $G(\mathbb{R})_{+}$and such that, on each copy of $\mathfrak{H}$ the element $\left(\begin{array}{rr}-1 & 0 \\ 0 & 1\end{array}\right)$ acts by $z \mapsto-\bar{z}$. We put $\underline{i}=(\sqrt{-1}, \ldots, \sqrt{-1}) \in \mathfrak{H}_{F}, K_{\infty}^{+}=\operatorname{Stab}_{G(\mathbb{R})_{+}}(\underline{i})=\mathrm{SO}_{2}(F \otimes \mathbb{R}) D(\mathbb{R})$ and $K_{\infty}=\operatorname{Stab}_{G(\mathbb{R})}(\underline{i})=\mathrm{O}_{2}(F \otimes \mathbb{R}) D(\mathbb{R})$.

We denote by $\widehat{\mathbb{Z}}=\prod_{l} \mathbb{Z}_{l}$ the profinite completion of $\mathbb{Z}$ and we put $\widehat{\mathfrak{o}}=\widehat{\mathbb{Z}} \otimes \mathfrak{o}=\prod_{v} \mathfrak{o}_{v}$, where $v$ runs over all the finite places of $F$. Let $\mathbb{A}$ (respectively $\mathbb{A}_{f}$ ) be the ring of adèles (respectively finite adèles) of $\mathbb{Q}$. We consider the following open compact subgroup of $G\left(\mathbb{A}_{f}\right)$ :

$$
K_{1}(\mathfrak{n})=\left\{\left(\begin{array}{ll}
a & b \\
c & d
\end{array}\right) \in G(\widehat{\mathbb{Z}}) \mid d-1 \in \mathfrak{n}, c \in \mathfrak{n}\right\} .
$$

The adélic Hilbert modular variety of level $K_{1}(\mathfrak{n})$ is defined as

$$
Y^{\mathrm{an}}=Y_{1}(\mathfrak{n})^{\mathrm{an}}=G(\mathbb{Q}) \backslash G(\mathbb{A}) / K_{1}(\mathfrak{n}) K_{\infty}^{+} .
$$

By the Strong Approximation Theorem, the connected components of $Y^{\text {an }}$ are indexed by the narrow ideal class group $\mathrm{Cl}_{F}^{+}=D(\mathbb{A}) / D(\mathbb{Q}) D(\widehat{\mathbb{Z}}) D(\mathbb{R})_{+}$of $F$. For each fractional ideal $\mathfrak{c}$ of $F$ we put $\mathfrak{c}^{*}=\mathfrak{c}^{-1} \mathfrak{d}^{-1}$. We define the following congruence subgroup of $G(\mathbb{Q})$ :

$$
\Gamma_{1}(\mathfrak{c}, \mathfrak{n})=\left\{\left(\begin{array}{ll}
a & b \\
c & d
\end{array}\right) \in G(\mathbb{Q}) \cap\left(\begin{array}{cc}
\mathfrak{o} & \mathfrak{c}^{*} \\
\mathfrak{c} \mathfrak{n} & \mathfrak{o}
\end{array}\right) \mid a d-b c \in \mathfrak{o}_{+}^{\times}, d \equiv 1(\bmod \mathfrak{n})\right\} .
$$

Put $M^{\text {an }}=M_{1}(\mathfrak{c}, \mathfrak{n})^{\text {an }}=\Gamma_{1}(\mathfrak{c}, \mathfrak{n}) \backslash \mathfrak{H}_{F}$. Then we have $Y_{1}(\mathfrak{n})^{\text {an }} \simeq \coprod_{i=1}^{h_{F}^{+}} M_{1}\left(\mathfrak{c}_{i}, \mathfrak{n}\right)^{\text {an }}$, where the ideals $\mathfrak{c}_{i}, 1 \leqslant i \leqslant h_{F}^{+}$, form a set of representatives of $\mathrm{Cl}_{F}^{+}$.

Put $\mathfrak{H}_{F}^{*}=\mathfrak{H}_{F} \coprod \mathbb{P}^{1}(F)$. The minimal compactification $M^{* \text { an }}$ of $M^{\text {an }}$ is defined as $M^{* \text { an }}=$ $\Gamma_{1}(\mathfrak{c}, \mathfrak{n}) \backslash \mathfrak{H}_{F}^{*}$. It is an analytic normal projective space whose boundary $M^{* \text { an }} \backslash M^{\text {an }}$ is a finite union of closed points, called the cusps of $M^{\text {an }}$.

The same way, by replacing $G$ by $G^{*}$, we define $\Gamma_{1}^{1}(\mathfrak{c}, \mathfrak{n}), M^{1, \text { an }}=M_{1}^{1}(\mathfrak{c}, \mathfrak{n})^{\text {an }}$ and $M^{1 * \text {,an }}$.

\subsection{Analytic Hilbert modular forms}

For the definition of the $\mathbb{C}$-vector space of Hilbert modular forms we follow [24].

$4^{\mathrm{e}}$ SÉRIE - TOME $38-2005-\mathrm{N}^{\circ} 4$ 
Definition 1.1. - An element $k=\sum_{\tau \in J_{F}} k_{\tau} \tau \in \mathbb{Z}\left[J_{F}\right]$ is called a weight. We always assume that the $k_{\tau}$ 's are $\geqslant 2$ and have the same parity. We put $k_{0}=\max \left\{k_{\tau} \mid \tau \in J_{F}\right\}$, $n_{0}=k_{0}-2, t=\sum_{\tau \in J_{F}} \tau, n=\sum_{\tau \in J_{F}} n_{\tau} \tau=k-2 t$ and $m=\sum_{\tau \in J_{F}} m_{\tau} \tau=\left(k_{0} t-k\right) / 2$.

For $z \in \mathfrak{H}_{F}, \gamma=\left(\begin{array}{ll}a & b \\ c & d\end{array}\right)$ we put $j_{J}(\gamma, z)=c \cdot z^{J}+d \in D(\mathbb{C})$, where

$$
z_{\tau}^{J}= \begin{cases}z_{\tau}, & \tau \in J \\ \bar{z}_{\tau}, & \tau \in J_{F} \backslash J\end{cases}
$$

DEFinition 1.2. - The space $G_{k, J}\left(K_{1}(\mathfrak{n})\right)$ of adélic Hilbert modular forms of weight $k$, level $K_{1}(\mathfrak{n})$ and type $J \subset J_{F}$ at infinity is the $\mathbb{C}$-vector space of the functions $g: G(\mathbb{A}) \rightarrow \mathbb{C}$ satisfying the following three conditions:

(i) $g($ axy $)=g(x)$ for all $a \in G(\mathbb{Q}), y \in K_{1}(\mathfrak{n})$ and $x \in G(\mathbb{A})$.

(ii) $g(x \gamma)=\nu(\gamma)^{k+m-t} j_{J}(\gamma, \underline{i})^{-k} g(x)$, for all $\gamma \in K_{\infty}^{+}$and $x \in G(\mathbb{A})$.

For all $x \in G\left(\mathbb{A}_{f}\right)$ define $g_{x}: \mathfrak{H}_{F} \rightarrow \mathbb{C}$, by $z \mapsto \nu(\gamma)^{t-k-m} j_{J}(\gamma, \underline{i})^{k} g(x \gamma)$, where $\gamma \in G(\mathbb{R})_{+}$ is such that $z=\gamma \cdot \underline{i}$. By (ii) $g_{x}$ does not depend on the particular choice of $\gamma$.

(iii) $g_{x}$ is holomorphic at $z_{\tau}$, for $\tau \in J$ and anti-holomorphic at $z_{\tau}$, for $\tau \in J_{F} \backslash J$ (when $F=\mathbb{Q}$ an extra condition of holomorphy at cusps is needed).

The space $S_{k, J}\left(K_{1}(\mathfrak{n})\right)$ of adélic Hilbert modular cuspforms is the subspace of $G_{k, J}\left(K_{1}(\mathfrak{n})\right)$ consisting of functions satisfying the following additional condition:

(iv) $\int_{U(\mathbb{Q}) \backslash U(\mathbb{A})} g(u x) d u=0$ for all $x \in G(\mathbb{A})$ and all additive Haar measures $d u$ on $U(\mathbb{A})$.

The conditions (i) and (ii) of the above definition imply that for all $g \in G_{k, J}\left(K_{1}(\mathfrak{n})\right)$ there exists a Hecke character $\psi$ of $F$ of conductor dividing $\mathfrak{n}$ and of type $-n_{0} t$ at infinity, such that for all $x \in G(\mathbb{A})$ and for all $z \in D(\mathbb{Q}) D(\widehat{\mathbb{Z}}) D(\mathbb{R})$, we have $g(z x)=\psi(z)^{-1} g(x)$.

DEFINITION 1.3. - Let $\psi$ be a Hecke character of $F$ of conductor dividing $\mathfrak{n}$ and of type $-n_{0} t$ at infinity. The space $S_{k, J}(\mathfrak{n}, \psi)$ (respectively $G_{k, J}(\mathfrak{n}, \psi)$ ) is defined as the subspace of $S_{k, J}\left(K_{1}(\mathfrak{n})\right)$ (respectively $G_{k, J}\left(K_{1}(\mathfrak{n})\right)$ ) of elements $g$ satisfying $g(z x)=\psi(z)^{-1} g(x)$ for all $x \in G(\mathbb{A})$ and for all $z \in D(\mathbb{A})$. When $J=J_{F}$ this space is denoted by $S_{k}(\mathfrak{n}, \psi)$ (respectively by $\left.G_{k}(\mathfrak{n}, \psi)\right)$.

Since the characters of the ideal class group $\mathrm{Cl}_{F}=D(\mathbb{A}) / D(\mathbb{Q}) D(\widehat{\mathbb{Z}}) D(\mathbb{R})$ of $F$ form a basis of the complex valued functions on this set, we have:

$$
G_{k, J}\left(K_{1}(\mathfrak{n})\right)=\bigoplus_{\psi} G_{k, J}(\mathfrak{n}, \psi), \quad S_{k, J}\left(K_{1}(\mathfrak{n})\right)=\bigoplus_{\psi} S_{k, J}(\mathfrak{n}, \psi)
$$

where $\psi$ runs over the Hecke characters of $F$ of conductor dividing $\mathfrak{n}$ and infinity type $-n_{0} t$. Let $\Gamma$ be a congruence subgroup of $G(\mathbb{Q})$. We recall the classical definition:

Definition 1.4. - The space $G_{k, J}(\Gamma ; \mathbb{C})$ of Hilbert modular forms of weight $k$, level $\Gamma$ and type $J \subset J_{F}$ at infinity is the $\mathbb{C}$-vector space of the functions $g: \mathfrak{H}_{F} \rightarrow \mathbb{C}$ which are holomorphic at $z_{\tau}$, for $\tau \in J$, anti-holomorphic at $z_{\tau}$, for $\tau \in J_{F} \backslash J$, and such that for every $\gamma \in \Gamma$ we have $g(\gamma(z))=\nu(\gamma)^{t-k-m} j_{J}(\gamma, z)^{k} g(z)$.

The space $S_{k, J}(\Gamma ; \mathbb{C})$ of Hilbert modular cuspforms is the subspace of $G_{k, J}(\Gamma ; \mathbb{C})$, consisting of functions vanishing at all cusps.

Put $x_{i}=\left(\begin{array}{cc}\eta_{i} & 0 \\ 0 & 1\end{array}\right)$, where $\eta_{i}$ is the idèle associated to the ideal $\mathfrak{c}_{i}, 1 \leqslant i \leqslant h_{F}^{+}$. The map $g \mapsto\left(g_{x_{i}}\right)_{1 \leqslant i \leqslant h_{F}^{+}}$(see Definition 1.2) induces isomorphisms: 


$$
\begin{aligned}
& G_{k, J}\left(K_{1}(\mathfrak{n})\right) \simeq \bigoplus_{1 \leqslant i \leqslant h_{F}^{+}} G_{k, J}\left(\Gamma_{1}\left(\mathfrak{c}_{i}, \mathfrak{n}\right) ; \mathbb{C}\right) \\
& S_{k, J}\left(K_{1}(\mathfrak{n})\right) \simeq \bigoplus_{1 \leqslant i \leqslant h_{F}^{+}} S_{k, J}\left(\Gamma_{1}\left(\mathfrak{c}_{i}, \mathfrak{n}\right) ; \mathbb{C}\right) .
\end{aligned}
$$

Let $d \mu(z)=\prod_{\tau \in J_{F}} y_{\tau}^{-2} d x_{\tau} d y_{\tau}$ be the standard Haar measure on $\mathfrak{H}_{F}$.

DEFINITION 1.5.-

(i) The Petersson inner product of two cuspforms $g, h \in S_{k, J}\left(K_{1}(\mathfrak{n})\right)$ is given by the formula

$$
(g, h)_{K_{1}(\mathfrak{n})}=\sum_{i=1}^{h_{F}^{+}} \int_{\Gamma_{1}\left(\mathfrak{c}_{i}, \mathfrak{n}\right) \backslash \mathfrak{H}_{F}} \overline{g_{i}(z)} h_{i}(z) y^{k} d \mu(z),
$$

where $\left(g_{i}\right)_{1 \leqslant i \leqslant h_{F}^{+}}$(respectively $\left(h_{i}\right)_{1 \leqslant i \leqslant h_{F}^{+}}$) is the image of $g$ (respectively $h$ ) under the isomorphism (2).

(ii) The Petersson inner product of two cuspforms $g, h \in S_{k, J}(\mathfrak{n}, \psi)$ is given by

$$
(g, h)_{\mathfrak{n}}=\int_{G(\mathbb{Q}) \backslash G(\mathbb{A}) / D(\mathbb{A}) K_{1}(\mathfrak{n}) K_{\infty}^{+}} \overline{g(x)} h(x)|\nu(x)|_{\mathbb{A}}^{-n_{0}} d \mu(x) .
$$

\subsection{Hilbert-Blumenthal Abelian varieties}

A sheaf over a scheme $S$ which is locally free of rank one over $\mathfrak{o} \otimes \mathcal{O}_{S}$, is called an invertible o-bundle on $S$.

DEFINITION 1.6. - A Hilbert-Blumenthal Abelian variety (HBAV) over a $\mathbb{Z}\left[\frac{1}{\mathrm{~N}_{F / \mathbb{Q}}(\mathfrak{d})}\right]$-scheme $S$ is an Abelian scheme $\pi: A \rightarrow S$ of relative dimension $d$ together with an injection $\mathfrak{o} \hookrightarrow \operatorname{End}(A / S)$, such that $\underline{\omega}_{A / S}:=\pi_{*} \Omega_{A / S}^{1}$ is an invertible o-bundle on $S$.

Let $\mathfrak{c}$ be a fractional ideal of $F$ and $\mathfrak{c}_{+}$be the cone of totally positive elements in $\mathfrak{c}$. Given a HBAV $A / S$, the functor assigning to a $S$-scheme $X$ the set $A(X) \otimes_{\mathfrak{o}} \mathfrak{c}$ is representable by another HBAV, denoted by $A \otimes_{\mathfrak{o}} \mathfrak{c}$. Then $\mathfrak{o} \hookrightarrow \operatorname{End}(A / S)$ yields $\mathfrak{c} \hookrightarrow \operatorname{Hom}_{\mathfrak{o}}\left(A, A \otimes_{\mathfrak{o}} \mathfrak{c}\right)$. The dual of a HBAV $A$ is denoted by $A^{t}$.

DEFINITION 1.7. -

(i) A c-polarization on a HBAV $A / S$ is an o-linear isomorphism $\lambda: A \otimes_{\mathfrak{o}} \mathfrak{c} \stackrel{\sim}{\longrightarrow} A^{t}$, such that under the induced isomorphism $\operatorname{Hom}_{\mathfrak{o}}\left(A, A \otimes_{\mathfrak{o}} \mathfrak{c}\right) \cong \operatorname{Hom}_{\mathfrak{o}}\left(A, A^{t}\right)$ elements of $\mathfrak{c}$ (respectively $\mathfrak{c}_{+}$) correspond exactly to symmetric elements (respectively polarizations).

(ii) A $\mathfrak{c}$-polarization class $\bar{\lambda}$ is an orbit of $\mathfrak{c}$-polarizations under $\mathfrak{o}_{+}^{\times}$.

Let $\left(\mathbb{G}_{m} \otimes \mathfrak{d}^{-1}\right)[\mathfrak{n}]$ be the reduced subscheme of $\mathbb{G}_{m} \otimes \mathfrak{d}^{-1}$, defined as the intersection of the kernels of multiplications by elements of $\mathfrak{n}$. Its Cartier dual is isomorphic to the finite group scheme $\mathfrak{o} / \mathfrak{n}$.

Definition 1.8. - A $\mu_{\mathfrak{n}}$-level structure on a $\mathrm{HBAV} A / S$ is an $\mathfrak{o}$-linear closed immersion $\alpha:\left(\mathbb{G}_{m} \otimes \mathfrak{d}^{-1}\right)[\mathfrak{n}] \hookrightarrow A$ of group schemes over $S$.

\subsection{Hilbert modular varieties}

We consider the contravariant functor $\underline{\mathcal{M}}^{1}$ (respectively $\underline{\mathcal{M}}$ ) from the category of $\mathbb{Z}\left[\frac{1}{\Delta}\right]$-schemes to the category of sets, assigning to a scheme $S$ the set of isomorphism classes

$4^{\text {e }}$ SÉRIE - TOME $38-2005-\mathrm{N}^{\circ} 4$ 
of triples $(A, \lambda, \alpha)$ (respectively $(A, \bar{\lambda}, \alpha)$ ) where $A$ is a HBAV over $S$ endowed with a $\mathfrak{c}$ polarization $\lambda$ (respectively a $\mathfrak{c}$-polarization class $\bar{\lambda}$ ) and a $\mu_{\mathfrak{n}}$-level structure $\alpha$. Assume the following condition:

(NT) $\mathfrak{n}$ does not divide 2 , nor 3 , nor $\mathrm{N}_{F / \mathbb{Q}}(\mathfrak{d})$.

Then $\Gamma_{1}(\mathfrak{c}, \mathfrak{n})$ is torsion free, and the functor $\underline{\mathcal{M}}^{1}$ is representable by a quasi-projective, smooth, geometrically connected $\mathbb{Z}\left[\frac{1}{\Delta}\right]$-scheme $M^{1}=M_{1}^{1}(\mathfrak{c}, \mathfrak{n})$ endowed with a universal HBAV $\pi: \mathcal{A} \rightarrow M^{1}$. By definition, the sheaf $\underline{\omega}_{\mathcal{A} / M^{1}}=\pi_{*} \Omega_{\mathcal{A} / M^{1}}^{1}$ is an invertible o-bundle on $M^{1}$. Consider the first de Rham cohomology sheaf $\mathcal{H}_{\mathrm{dR}}^{1}\left(\mathcal{A} / M^{1}\right)=R^{1} \pi_{*} \Omega_{\mathcal{A} / M^{1}}$ on $M^{1}$. The Hodge filtration yields an exact sequence:

$$
0 \rightarrow \underline{\omega}_{\mathcal{A} / M^{1}} \rightarrow \mathcal{H}_{\mathrm{dR}}^{1}\left(\mathcal{A} / M^{1}\right) \rightarrow \underline{\omega}_{\mathcal{A} / M^{1}}^{\vee} \otimes \mathfrak{c d}^{-1} \rightarrow 0 .
$$

Therefore $\mathcal{H}_{\mathrm{dR}}^{1}\left(\mathcal{A} / M^{1}\right)$ is locally free of rank two over $\mathfrak{o} \otimes \mathcal{O}_{M^{1}}$.

The functor $\underline{\mathcal{M}}$ admits a coarse moduli space $M=M_{1}(\mathfrak{c}, \mathfrak{n})$, which is a quasi-projective, smooth, geometrically connected $\mathbb{Z}\left[\frac{1}{\Delta}\right]$-scheme. The finite group $\mathfrak{o}_{+}^{\times} / \mathfrak{o}_{\mathfrak{n}, 1}^{\times 2}$ acts properly and discontinuously on $M^{1}$ by $[\epsilon]:(A, \lambda, \alpha) / S \mapsto(A, \epsilon \lambda, \alpha) / S$ and the quotient is given by $M$. This group acts also on $\underline{\omega}_{\mathcal{A} / M^{1}}$ and on $\mathcal{H}_{\mathrm{dR}}^{1}\left(\mathcal{A} / M^{1}\right)$ by acting on the de Rham complex $\Omega_{\mathcal{A} / M^{1}}^{\bullet}$ ( $[\epsilon]$ acts on $\underline{\omega}_{\mathcal{A} / M^{1}}$ by $\left.\epsilon^{-1 / 2}[\epsilon]^{*}\right)$.

These actions are defined over the ring of integers of the number field $F\left(\epsilon^{1 / 2}, \epsilon \in \mathfrak{o}_{+}^{\times}\right)$.

Let $\mathfrak{o}^{\prime}$ be the ring of integers of $\widetilde{F}\left(\epsilon^{1 / 2}, \epsilon \in \mathfrak{o}_{+}^{\times}\right)$. For every $\mathbb{Z}\left[\frac{1}{\Delta}\right]$-scheme $S$ we put

$$
S^{\prime}=S \times \operatorname{Spec}\left(\mathfrak{o}^{\prime}\left[\frac{1}{\Delta}\right]\right)
$$

The sheaf of $\mathfrak{o}_{+}^{\times} / \mathfrak{o}_{\mathfrak{n}, 1}^{\times 2}$-invariants of $\underline{\omega}_{\mathcal{A} / M^{1}}$ (respectively of $\mathcal{H}_{\mathrm{dR}}^{1}\left(\mathcal{A} / M^{1}\right)$ ) is locally free of rank one (respectively two) over $\mathfrak{o} \otimes \mathcal{O}_{M^{\prime}}$ and is denoted by $\underline{\omega}$ (respectively $\mathcal{H}_{\mathrm{dR}}^{1}$ ).

We put $Y=Y_{1}(\mathfrak{n})=\coprod_{i=1}^{h_{F}^{+}} M_{1}\left(\mathfrak{c}_{i}, \mathfrak{n}\right)$ and $Y^{1}=Y_{1}^{1}(\mathfrak{n})=\coprod_{i=1}^{h_{F}^{+}} M_{1}^{1}\left(\mathfrak{c}_{i}, \mathfrak{n}\right)$, where the ideals $\mathfrak{c}_{i}, 1 \leqslant i \leqslant h_{F}^{+}$, form a set of representatives of $\mathrm{Cl}_{F}^{+}$.

\subsection{Geometric Hilbert modular forms}

Under the action of $\mathfrak{o}$, the invertible o-bundle $\underline{\omega}$ on $M^{\prime}$ decomposes as a direct sum of line bundles $\underline{\omega}_{\tau}, \tau \in J_{F}$. For every $k=\sum_{\tau} k_{\tau} \tau \in \mathbb{Z}\left[\bar{J}_{F}\right]$ we define the line bundle $\underline{\omega}^{k}=\bigotimes_{\tau} \underline{\omega}_{\tau}^{\otimes k_{\tau}}$ on $M^{\prime}$.

One should be careful to observe, that the global section of $\underline{\omega}^{k}$ on $M^{\text {an }}$ is given by the cocycle $\gamma \mapsto \nu(\gamma)^{-k / 2} j(\gamma, z)^{k}$, meanwhile we are interested in finding a geometric interpretation of the cocycle $\gamma \mapsto \nu(\gamma)^{t-k-m} j(\gamma, z)^{k}$ used in Definition 1.4.

The universal polarization class $\bar{\lambda}$ endows $\mathcal{H}_{\mathrm{dR}}^{1}$ with a perfect symplectic o-linear pairing. Consider the invertible o-bundle $\underline{\nu}:=\wedge_{\mathfrak{o} \otimes \mathcal{O}_{M^{\prime}}}^{2} \mathcal{H}_{\mathrm{dR}}^{1}$ on $M^{\prime}$. Note that $(k+m-t)-\frac{k}{2}=\frac{n_{0}}{2} t$.

Definition 1.9. - Let $R$ be an $\mathfrak{o}^{\prime}\left[\frac{1}{\Delta}\right]$-algebra. A Hilbert modular form of weight $k$, level $\Gamma_{1}(\mathfrak{c}, \mathfrak{n})$ and coefficients in $R$ is a global section of $\underline{\omega}^{k} \otimes \underline{\nu}^{-n_{0} t / 2}$ over $M \times_{\operatorname{Spec}\left(\mathbb{Z}\left[\frac{1}{\Delta}\right]\right)} \operatorname{Spec}(R)$. We denote by $G_{k}\left(\Gamma_{1}(\mathfrak{c}, \mathfrak{n}) ; R\right)=\mathrm{H}^{0}\left(M \times_{\operatorname{Spec}\left(\mathbb{Z}\left[\frac{1}{\Delta}\right]\right)} \operatorname{Spec}(R), \underline{\omega}^{k} \otimes \underline{\nu}^{-n_{0} t / 2}\right)$ the $R$-module of these Hilbert modular forms. 


\subsection{Toroidal compactifications}

The toroidal compactifications of the moduli space of $\mathfrak{c}$-polarized HBAV with principal level structure have been constructed by Rapoport [34]. Several modifications need to be made in order to treat the case of $\mu_{\mathfrak{n}}$-level structure. These are described in [10, Theorem 7.2].

Let $\Sigma$ be a smooth $\Gamma_{1}^{1}(\mathfrak{c}, \mathfrak{n})$-admissible collection of fans (see [10, Definition 7.1]). Then, there exists an open immersion of $M^{1}$ into a proper and smooth $\mathbb{Z}\left[\frac{1}{\Delta}\right]$-scheme $\overline{M^{1}}=M_{\Sigma}^{1}$, called the toroidal compactification of $M^{1}$ with respect to $\Sigma$. The universal HBAV $\pi: \mathcal{A} \rightarrow M^{1}$ extends uniquely to a semi-Abelian scheme $\bar{\pi}: \mathfrak{G} \rightarrow \overline{M^{1}}$. The group scheme $\mathfrak{G}$ is endowed with an action of $\mathfrak{o}$ and its restriction to $\overline{M^{1}} \backslash M^{1}$ is a torus. Moreover, the sheaf $\underline{\omega}_{\mathfrak{G} / \overline{M^{1}}}$ of $\mathfrak{G}$-invariants sections of $\bar{\pi}_{*} \Omega_{\mathfrak{G} / \overline{M^{1}}}^{1}$ is an invertible o-bundle on $\overline{M^{1}}$ extending $\underline{\omega}_{\mathcal{A} / M^{1}}$.

The scheme $\overline{M^{1}} \backslash M^{1}$ is a divisor with normal crossings and the formal completion of $\overline{M^{1}}$ along this divisor can be completely determined in terms of $\Sigma$ (see [10, Theorem 7.2]). For the sake of simplicity, we will only describe the completion of $\overline{M^{1}}$ along the connected component of $\overline{M^{1}} \backslash M^{1}$ corresponding to the standard cusp at $\infty$. Let $\Sigma^{\infty} \in \Sigma$ be the fan corresponding to the cusp at $\infty$. It is a complete, smooth fan of $\mathfrak{c}_{+}^{*} \cup\{0\}$, stable by the action of $\mathfrak{o}_{\mathfrak{n}, 1}^{\times 2}$, and containing a finite number of cones modulo this action. Put $R_{\infty}=\mathbb{Z}\left[q^{\xi}, \xi \in \mathfrak{c}\right]$ and $S_{\infty}=\operatorname{Spec}\left(R_{\infty}\right)=$ $\mathbb{G}_{m} \otimes \mathfrak{c}^{*}$. Associated to the fan $\Sigma^{\infty}$, there is a toroidal embedding $S_{\infty} \hookrightarrow S_{\Sigma^{\infty}}$ (it is obtained by gluing the affine toric embeddings $S_{\infty} \hookrightarrow S_{\infty, \sigma}=\operatorname{Spec}\left(\mathbb{Z}\left[q^{\xi}, \xi \in \mathfrak{c} \cap \check{\sigma}\right]\right)$ for $\left.\sigma \in \Sigma^{\infty}\right)$. Let $S_{\Sigma \infty}^{\wedge}$ be the formal completion of $S_{\Sigma^{\infty}}$ along $S_{\Sigma^{\infty}} \mid S_{\infty}$. By construction, the formal completion of $\overline{M^{1}}$ along the connected component of $\overline{M^{1}} \backslash M^{1}$ corresponding to the standard cusp at $\infty$ is isomorphic to $S_{\Sigma \infty}^{\wedge} / \mathfrak{o}_{\mathfrak{n}, 1}^{\times 2}$.

Assume that $\Sigma$ is $\Gamma_{1}(\mathfrak{c}, \mathfrak{n})$-admissible (for the cusp at $\infty$ it means that $\Sigma^{\infty}$ is stable under the action of $\left.\mathfrak{o}_{+}^{\times}\right)$. Then the finite group $\mathfrak{o}_{+}^{\times} / \mathfrak{o}_{\mathfrak{n}, 1}^{\times 2}$ acts properly and discontinuously on $\overline{M^{1}}$ and the quotient $\bar{M}=M_{\Sigma}$ is a proper and smooth $\mathbb{Z}\left[\frac{1}{\Delta}\right]$-scheme, containing $M$ as an open subscheme. Again by construction, the formal completion of $\bar{M}$ along the connected component of $\bar{M} \backslash M$ corresponding to the standard cusp at $\infty$ is isomorphic to $S_{\Sigma_{\infty}}^{\wedge} / \mathfrak{o}_{+}^{\times}$.

The invertible o-bundle $\underline{\omega}_{\mathfrak{G} / \overline{M^{1}}}$ on $\overline{M^{1}}$ descends to an invertible o-bundle on $\bar{M}^{\prime}$, extending $\underline{\omega}$. We still denote this extension by $\underline{\omega}$. For each $k \in \mathbb{Z}\left[J_{F}\right]$ this gives us an extension of $\underline{\omega}^{k}$ to a line bundle on $\bar{M}^{\prime}$, still denoted by $\underline{\omega}^{k}$.

\section{7. $q$-expansion and Koecher Principles}

If $F \neq \mathbb{Q}$ the Koecher Principle states that

$$
\mathrm{H}^{0}\left(M \times \operatorname{Spec}(R), \underline{\omega}^{k} \otimes \underline{\nu}^{-n_{0} t / 2}\right)=\mathrm{H}^{0}\left(\bar{M} \times \operatorname{Spec}(R), \underline{\omega}^{k} \otimes \underline{\nu}^{-n_{0} t / 2}\right) .
$$

For a proof we refer to [10, Theorem 8.3]. For simplicity, we will only describe the $q$-expansion at the standard (unramified) cusp at $\infty$. For every $\sigma \in \Sigma^{\infty}$ and every $\mathfrak{o}^{\prime}\left[\frac{1}{\Delta}\right]$-algebra $R$, the pullback of $\underline{\omega}$ to $S_{\Sigma \infty}^{\wedge} \times \operatorname{Spec}(R)$ is canonically isomorphic to $\mathfrak{o} \otimes \mathcal{O}_{S_{\Sigma}^{\wedge}} \otimes R$. Thus

$$
\begin{aligned}
& \mathrm{H}^{0}\left(S_{\Sigma^{\infty}}^{\wedge} \times \operatorname{Spec}(R) / \mathfrak{o}_{+}^{\times}, \underline{\omega}^{k} \otimes \underline{\nu}^{-n_{0} t / 2}\right) \\
& \quad=\left\{\sum_{\xi \in \mathfrak{c}_{+} \cup\{0\}} a_{\xi} q^{\xi} \mid a_{\xi} \in R, a_{u^{2} \epsilon \xi}=u^{k} \epsilon^{k+m-t} a_{\xi}, \forall(u, \epsilon) \in \mathfrak{o}_{\mathfrak{n}, 1}^{\times} \times \mathfrak{o}_{+}^{\times}\right\} .
\end{aligned}
$$

This construction associates to each $g \in G_{k}\left(\Gamma_{1}(\mathfrak{c}, \mathfrak{n}) ; R\right)$ an element $g_{\infty}=\sum_{\xi \in \mathfrak{c}_{+} \cup\{0\}} a_{\xi}(g) q^{\xi}$, called the $q$-expansion of $g$ at the cusp at $\infty$. The element $a_{0}(g) \in R$ is the value of $g$ at the cusp at $\infty$.

$4^{\mathrm{e}}$ SÉRIE - TOME $38-2005-\mathrm{N}^{\circ} 4$ 
PROPOSITION 1.10. - Let $R$ be a $\mathfrak{o}^{\prime}\left[\frac{1}{\Delta}\right]$-algebra.

(i) (q-expansion Principle) $G_{k}\left(\Gamma_{1}(\mathfrak{c}, \mathfrak{n}) ; R\right) \rightarrow R\left[\left[q^{\xi}, \xi \in \mathfrak{c}_{+} \cup\{0\}\right]\right], g \mapsto g_{\infty}$ is injective.

(ii) If there exists $g \in G_{k}\left(\Gamma_{1}(\mathfrak{c}, \mathfrak{n}) ; R\right)$ such that $a_{0}(g) \neq 0$, then $\epsilon^{k+m-t}-1$ is a zero-divisor in $R$, for all $\epsilon \in \mathfrak{o}_{+}^{\times}$.

\subsection{The minimal compactification}

There exists a projective, normal $\mathbb{Z}\left[\frac{1}{d}\right]$-scheme $M^{1 *}$, containing $M^{1}$ as an open dense subscheme and such that the scheme $M^{1 *} \backslash M^{1}$ is finite and étale over $\mathbb{Z}\left[\frac{1}{\Delta}\right]$. Moreover, for each toroidal compactification $\overline{M^{1}}$ of $M^{1}$ there is a natural surjection $\overline{M^{1}} \rightarrow M^{1 *}$ inducing the identity map on $M^{1}$. The scheme $M^{1 *}$ is called the minimal compactification of $M^{1}$. The action of $\mathfrak{o}_{+}^{\times} / \mathfrak{o}_{\mathfrak{n}, 1}^{\times 2}$ on $M^{1}$ extends to an action on $M^{1 *}$ and the minimal compactification $M^{*}$ of $M$ is defined as the quotient for this action. In general $M^{1 *} \rightarrow M^{*}$ is not étale.

We summarize the above discussion in the following commutative diagram:

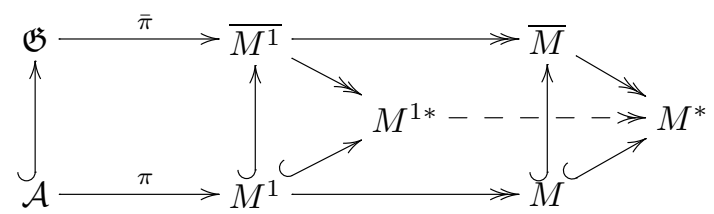

\subsection{Toroidal compactifications of Kuga-Sato varieties}

Let $s$ be a positive integer. Let $\pi_{s}: \mathcal{A}^{s} \rightarrow M^{1}$ be the $s$-fold fiber product of $\pi: \mathcal{A} \rightarrow M^{1}$ and $(\bar{\pi})_{s}: \mathfrak{G}^{s} \rightarrow \overline{M^{1}}$ be the $s$-fold fiber product of $\bar{\pi}: \mathfrak{G} \rightarrow \overline{M^{1}}$.

Let $\widetilde{\Sigma}$ be a $(\mathfrak{o} \oplus \mathfrak{c}) \rtimes \Gamma_{1}^{1}(\mathfrak{c}, \mathfrak{n})$-admissible, polarized, equidimensional, smooth collection of fans, above the $\Gamma_{1}^{1}(\mathfrak{c}, \mathfrak{n})$-admissible collection of fans $\Sigma$ of Section 1.6. Using Faltings-Chai's method [15], the main result of [11, Section 6] is the following: there exists an open immersion of a $\mathcal{A}^{s}$ into a projective smooth $\mathbb{Z}\left[\frac{1}{\Delta}\right]$-scheme $\overline{\mathcal{A}^{s}}=\mathcal{A}_{\widetilde{\Sigma}}^{s}$, and a proper, semi-stable homomorphism

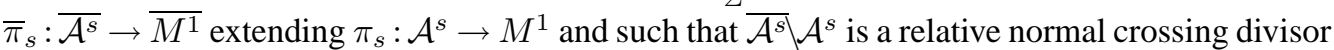
above $\overline{M^{1}} \backslash M^{1}$. Moreover, $\overline{\mathcal{A}^{s}}$ contains $\mathfrak{G}^{s}$ as an open dense subscheme and $\mathfrak{G}^{s}$ acts on $\overline{\mathcal{A}^{s}}$ extending the translation action of $\mathcal{A}^{s}$ on itself.

The sheaf $\mathcal{H}_{\log -\mathrm{dR}}^{1}\left(\overline{\mathcal{A}} / \overline{M^{1}}\right)=R^{1} \overline{\pi_{1} *} \Omega_{\overline{\mathcal{A}} / \overline{M^{1}}}^{\bullet}(\mathrm{d} \log \infty)$ is independent of the particular choice of $\widetilde{\Sigma}$ above $\Sigma$ and is endowed with a filtration:

$$
0 \rightarrow \underline{\omega}_{\mathfrak{G} / \overline{M^{1}}} \rightarrow \mathcal{H}_{\log -\mathrm{dR}}^{1}\left(\overline{\mathcal{A}} / \overline{M^{1}}\right) \rightarrow \underline{\omega}_{\mathfrak{G} / \overline{M^{1}}}^{\vee} \otimes \mathfrak{c d}^{-1} \rightarrow 0 .
$$

It descends to a sheaf $\mathcal{H}_{\text {log-dR }}^{1}$ on $\bar{M}$ which fits in the following exact sequence:

$$
0 \rightarrow \underline{\omega} \rightarrow \mathcal{H}_{\log -\mathrm{dR}}^{1} \rightarrow \underline{\omega}^{\vee} \otimes \mathfrak{c d}^{-1} \rightarrow 0 .
$$

\subsection{Hecke operators on modular forms}

Let $\mathbb{Z}\left[K_{1}(\mathfrak{n}) \backslash G\left(\mathbb{A}_{f}\right) / K_{1}(\mathfrak{n})\right]$ be the free Abelian group with basis the double cosets of $K_{1}(\mathfrak{n})$ in $G\left(\mathbb{A}_{f}\right)$. It is endowed with algebra structure, where the product of two basis elements is given by:

$$
\left[K_{1}(\mathfrak{n}) x K_{1}(\mathfrak{n})\right] \cdot\left[K_{1}(\mathfrak{n}) y K_{1}(\mathfrak{n})\right]=\sum_{i}\left[K_{1}(\mathfrak{n}) x_{i} y K_{1}(\mathfrak{n})\right]
$$


where $\left[K_{1}(\mathfrak{n}) x K_{1}(\mathfrak{n})\right]=\coprod_{i} K_{1}(\mathfrak{n}) x_{i}$. For $g \in S_{k, J}\left(K_{1}(\mathfrak{n})\right)$ we put:

$$
\left.g\right|_{\left[K_{1}(\mathfrak{n}) x K_{1}(\mathfrak{n})\right]}(\cdot)=\sum_{i} g\left(\cdot x_{i}^{-1}\right) .
$$

This defines an action of the algebra $\mathbb{Z}\left[K_{1}(\mathfrak{n}) \backslash G\left(\mathbb{A}_{f}\right) / K_{1}(\mathfrak{n})\right]$ on $S_{k, J}\left(K_{1}(\mathfrak{n})\right)$ (respectively on $G_{k, J}\left(K_{1}(\mathfrak{n})\right)$ ). Since this algebra is not commutative when $\mathfrak{n} \neq \mathfrak{o}$, we will define a commutative subalgebra. Consider the semi-group:

$$
\Delta(\mathfrak{n})=\left\{\left(\begin{array}{ll}
a & b \\
c & d
\end{array}\right) \in G\left(\mathbb{A}_{f}\right) \cap \mathrm{M}_{2}(\hat{\mathfrak{o}}) \mid d_{v} \in \mathfrak{o}_{v}^{\times}, c_{v} \in \mathfrak{n}_{v} \text { for all } v \text { dividing } \mathfrak{n}\right\} .
$$

The abstract Hecke algebra of level $K_{1}(\mathfrak{n})$ is defined as $\mathbb{Z}\left[K_{1}(\mathfrak{n}) \backslash \Delta(\mathfrak{n}) / K_{1}(\mathfrak{n})\right]$ endowed with the convolution product (4). This algebra has the following explicit description.

For each ideal $\mathfrak{a} \subset \mathfrak{o}$ we define the Hecke operator $T_{\mathfrak{a}}$ as the finite sum of double cosets $\left[K_{1}(\mathfrak{n}) x K_{1}(\mathfrak{n})\right]$ contained in the set $\{x \in \Delta(\mathfrak{n}) \mid \nu(x) \mathfrak{o}=\mathfrak{a}\}$. In the same way, for an ideal $\mathfrak{a} \subset \mathfrak{o}$ which is prime to $\mathfrak{n}$, we define the Hecke operator $S_{\mathfrak{a}}$ by the double coset for $K_{1}(\mathfrak{n})$ containing the scalar matrix of the idèle attached to the ideal $\mathfrak{a}$.

For each finite place $v$ of $F$, we have $T_{v}=K_{1}(\mathfrak{n})\left(\begin{array}{cc}\varpi_{v} & 0 \\ 0 & 1\end{array}\right) K_{1}(\mathfrak{n})$ and for each $v$ not dividing $\mathfrak{n}$ we have $S_{v}=K_{1}(\mathfrak{n})\left(\begin{array}{cc}\varpi_{v} & 0 \\ 0 & \varpi_{v}\end{array}\right) K_{1}(\mathfrak{n})$, where $\varpi_{v}$ is an uniformizer of $F_{v}$.

Then, the abstract Hecke algebra of level $K_{1}(\mathfrak{n})$ is isomorphic to the polynomial algebra in the variables $T_{v}$, where $v$ runs over the prime ideals of $F$, and the variables $S_{v}^{ \pm 1}$, where $v$ runs over the prime ideals of $F$ not dividing $\mathfrak{n}$. The action of Hecke algebra obviously preserves the decomposition (1) and moreover, $S_{v}$ acts on $S_{k, J}(\mathfrak{n}, \psi)$ as the scalar $\psi(v)$.

Let $\mathbb{T}(\mathbb{C})$ be the subalgebra of $\operatorname{End}_{\mathbb{C}}\left(S_{k, J}\left(K_{1}(\mathfrak{n})\right)\right)$ generated by the operators $S_{v}$ for $v \nmid \mathfrak{n}$ and $T_{v}$ for all $v$ (we will see in Section 1.13 that $\mathbb{T}(\mathbb{C})$ does not depend on $J$ ).

The algebra $\mathbb{T}(\mathbb{C})$ is commutative, but not semi-simple in general. Nevertheless, for $v \nmid \mathfrak{n}$ the operators $S_{v}$ and $T_{v}$ are normal with respect to the Petersson inner product (see Definition 1.5). Denote by $\mathbb{T}^{\prime}(\mathbb{C})$ the subalgebra of $\mathbb{T}(\mathbb{C})$ generated by the Hecke operators outside a finite set of places containing those dividing $\mathfrak{n}$. The algebra $\mathbb{T}^{\prime}(\mathbb{C})$ is semi-simple, that is to say $S_{k, J}\left(K_{1}(\mathfrak{n})\right)$ has a basis of eigenvectors for $\mathbb{T}^{\prime}(\mathbb{C})$.

We will now describe the relation between Fourier coefficients and eigenvalues for the Hecke operators. By (2) we can associate to $g \in S_{k}\left(K_{1}(\mathfrak{n})\right)$ a family of classical cuspforms $g_{i} \in S_{k}\left(\Gamma_{1}\left(\mathfrak{c}_{i}, \mathfrak{n}\right) ; \mathbb{C}\right)$, where $\mathfrak{c}_{i}$ are representatives of the narrow ideal class group $\mathrm{Cl}_{F}^{+}$.

Each form $g_{i}$ is determined by its $q$-expansion at the cusp $\infty$ of $M_{1}\left(\mathfrak{c}_{i}, \mathfrak{n}\right)^{\text {an }}$. For each fractional ideal $\mathfrak{a}=\mathfrak{c}_{i} \xi$, with $\xi \in F_{+}^{\times}$, we put $c(g, \mathfrak{a})=\xi^{m} a_{\xi}\left(g_{i}\right)$. By Section 1.7 for each $\epsilon \in \mathfrak{o}_{+}^{\times}$, we have $a_{\epsilon \xi}=\epsilon^{k+m-t} a_{\xi}$ and therefore the definition of $c(g, \mathfrak{a})$ does not depend on the choice of $\xi$ (nor on the particular choice of the ideals $\mathfrak{c}_{i}$; see [20, IV.4.2.9]).

DEFINITION 1.11. - We say that $g \in S_{k}\left(K_{1}(\mathfrak{n})\right)$ is an eigenform, if it is an eigenvector for $\mathbb{T}(\mathbb{C})$. In this case $g \in S_{k}(\mathfrak{n}, \psi)$ for some Hecke character $\psi$, called the central character of $g$. We say that an eigenform $g$ is normalized if $c(g, \mathfrak{o})=1$.

Lemma 1.12 ([24, Proposition 4.1, Theorem 5.2], [20, (4.64)]). - If $g \in S_{k}\left(K_{1}(\mathfrak{n})\right)$ is a normalized eigenform, then the eigenvalue of $T_{\mathfrak{a}}$ on $g$ is equal to the Fourier coefficient $c(g, \mathfrak{a})$.

The pairing $\mathbb{T}(\mathbb{C}) \times S_{k}\left(K_{1}(\mathfrak{n})\right) \rightarrow \mathbb{C},(T, g) \mapsto c\left(\left.g\right|_{T}, \mathfrak{o}\right)$ is a perfect duality.

A consequence of this lemma and the $q$-expansion Principle (see Section 1.7) is the Weak Multiplicity One Theorem stating that two normalized eigenforms having the same eigenvalues are equal.

$4^{\text {e }}$ SÉRIE - TOME $38-2005-\mathrm{N}^{\circ} 4$ 


\subsection{Ordinary modular forms}

When the weight $k$ is non-parallel, the definition of the Hecke operators should be slightly modified. We put $T_{0, v}=\varpi_{v}^{-m} T_{v}$ and $S_{0, v}=\varpi_{v}^{-2 m} S_{v}$ (see [24, Section 3]; in the applications our base ring will be the $p$-adic ring $\mathcal{O}$ which satisfies the assumptions of this reference).

The advantage of the Hecke operators $T_{0, v}$ and $S_{0, v}$ is that they preserve in an optimal way the $\mathcal{O}$-integral structures on the space of Hilbert modular forms and on the cohomology of the Hilbert modular variety.

DEFINITION 1.13. - A normalized Hilbert modular eigenform is ordinary at $p$ if for all primes $\mathfrak{p}$ of $F$ dividing $p$, the image by $\iota_{p}$ of its $T_{0, \mathfrak{p}}$-eigenvalue is a $p$-adic unit.

\subsection{Primitive modular forms}

For each $\mathfrak{n}_{1}$ dividing $\mathfrak{n}$ and divisible by the conductor of $\psi$, and for all $\mathfrak{n}_{2}$ dividing $\mathfrak{n n}_{1}^{-1}$ we consider the linear map

$$
S_{k}\left(\mathfrak{n}_{1}, \psi\right) \rightarrow S_{k}(\mathfrak{n}, \psi), \quad g \mapsto g \mid \mathfrak{n}_{2},
$$

where $g \mid \mathfrak{n}_{2}$ is determined by the relation $c\left(\mathfrak{a}, g \mid \mathfrak{n}_{2}\right)=c\left(\mathfrak{a n n}_{2}^{-1}, g\right)$.

We define the subspace $S_{k}^{\text {old }}(\mathfrak{n}, \psi)$ of $S_{k}(\mathfrak{n}, \psi)$ as the subspace generated by the images of all these linear maps. This space is preserved by the Hecke operators outside $\mathfrak{n}$. We define the space $S_{k}^{\text {new }}(\mathfrak{n}, \psi)$ of the primitive modular forms as the orthogonal of $S_{k}^{\text {old }}(\mathfrak{n}, \psi)$ in $S_{k}(\mathfrak{n}, \psi)$ with respect to the Petersson inner product (see Definition 1.5). Since the Hecke operators outside $\mathfrak{n}$ are normal for the Petersson inner product, the direct sum decomposition $S_{k}(\mathfrak{n}, \psi)=$ $S_{k}^{\text {new }}(\mathfrak{n}, \psi) \oplus S_{k}^{\text {old }}(\mathfrak{n}, \psi)$ is preserved by $\mathbb{T}^{\prime}(\mathbb{C})$. The Strong Multiplicity One Theorem, due to Miyake in the Hilbert modular case, asserts that if $f \in S_{k}^{\text {new }}(\mathfrak{n}, \psi)$ is an eigenform for $\mathbb{T}^{\prime}(\mathbb{C})$, then it is an eigenform for $\mathbb{T}(\mathbb{C})$.

A normalized primitive eigenform is called a newform.

\subsection{External and Weyl group conjugates}

For an element $\sigma \in \operatorname{Aut}(\mathbb{C})$ we define the external conjugate of $g \in S_{k}\left(K_{1}(\mathfrak{n})\right)$ as the unique element $g^{\sigma} \in S_{k}\left(K_{1}(\mathfrak{n})\right)$ satisfying $c\left(g^{\sigma}, \mathfrak{a}\right)=c(g, \mathfrak{a})^{\sigma}$ for each ideal $\mathfrak{a}$ of $\mathfrak{o}$.

We identify $\{ \pm 1\}^{J_{F}}$ with the Weyl group $K_{\infty} / K_{\infty}^{+}$of $G$ by sending $\epsilon_{J}=\left(-1_{J}, 1_{J_{F} \backslash J}\right)$ to $c_{J} K_{\infty}^{+}$, where for all $\tau \in J_{F}$, $\operatorname{det}\left(c_{J, \tau}\right)<0$ if and only if $\tau \in J$. The length of $\epsilon_{J}$ is $|J|$.

We have an action of the Weyl group on the space of Hilbert modular forms. More precisely, $\epsilon_{J}$ acts as the double class $\left[K_{1}(\mathfrak{n}) c_{J} K_{1}(\mathfrak{n})\right]$ and maps bijectively $S_{k}\left(K_{1}(\mathfrak{n})\right)$ onto $S_{k, J_{F} \backslash J}\left(K_{1}(\mathfrak{n})\right)$. The action of $\epsilon_{J}$ commutes with the action of the Hecke operators. For an element $g \in S_{k}\left(K_{1}(\mathfrak{n})\right)$ we put $g_{J}=\epsilon_{J_{F} \backslash J} \cdot g$.

\subsection{Eichler-Shimura-Harder isomorphism}

Let $R$ be an $\mathcal{O}$-algebra and let $V_{n}(R)$ be the polynomial ring over $R$ in the variables $\left(X_{\tau}, Y_{\tau}\right)_{\tau \in J_{F}}$ which are homogeneous of degree $n_{\tau}$ in $\left(X_{\tau}, Y_{\tau}\right)$. We have a pairing (perfect if $n_{0}$ ! is invertible in $R$ )

$$
\begin{aligned}
& \langle,\rangle: V_{n}(R) \times V_{n}(R) \rightarrow R, \text { given by } \\
& \left\langle\sum_{0 \leqslant j \leqslant n} a_{j} X^{n-j} Y^{j}, \sum_{0 \leqslant j \leqslant n} b_{j} X^{n-j} Y^{j}\right\rangle=\sum_{0 \leqslant j \leqslant n}(-1)^{j} a_{j} b_{n-j}\left(\begin{array}{c}
n \\
j
\end{array}\right), \\
& \text { where }\left(\begin{array}{c}
n \\
j
\end{array}\right)=\prod_{\tau \in J_{F}}\left(\begin{array}{c}
n_{\tau} \\
j_{\tau}
\end{array}\right) .
\end{aligned}
$$


The $R$-module $V_{n}(R)$ realizes the algebraic representation $V_{n}=\bigotimes_{\tau}\left(\operatorname{Sym}^{n_{\tau}} \otimes \operatorname{det}^{m_{\tau}}\right)$ of $G(R)$. We endow $V_{n}(R)$ with an action of $\left(M_{2}(\mathcal{O}) \cap \mathrm{GL}_{2}(E)\right)^{J_{F}}$ given by

$$
\gamma \cdot P\left(\left(X_{\tau}, Y_{\tau}\right)_{\tau \in J_{F}}\right)=\nu(\gamma)^{m} P\left(\left(\operatorname{det}(\gamma) \gamma^{-1}\right)^{t}\left(X_{\tau}, Y_{\tau}\right)_{\tau \in J_{F}}\right)
$$

Let $\mathbb{V}_{n}(R)$ be the sheaf of continuous (thus locally constant) sections of

$$
G(\mathbb{Q}) \backslash G(\mathbb{A}) \times V_{n}(R) / K_{1}(\mathfrak{n}) K_{\infty}^{+} \rightarrow G(\mathbb{Q}) \backslash G(\mathbb{A}) / K_{1}(\mathfrak{n}) K_{\infty}^{+}=Y^{\text {an }}
$$

where $y \in K_{1}(\mathfrak{n}) K_{\infty}^{+}$acts on $V_{n}(R)$ via its $p$-part $y_{p}$

For each $y \in \Delta(\mathfrak{n})$ the map $[y]: G(\mathbb{A}) \times V_{n}(R) \rightarrow G(\mathbb{A}) \times V_{n}(R),(x, v) \mapsto\left(x y, y_{p} \cdot v\right)$ is a homomorphism of sheaves. This induces an action of the Hecke operator $\left[K_{1}(\mathfrak{n}) y K_{1}(\mathfrak{n})\right]$ on $\mathrm{H}^{d}\left(Y^{\mathrm{an}}, \mathbb{V}_{n}(R)\right)$ preserving the cuspidal cohomology $\mathrm{H}_{\text {cusp }}^{d}\left(Y^{\mathrm{an}}, \mathbb{V}_{n}(R)\right)$.

The action of $\epsilon_{J}$ on $\left(M^{\mathrm{an}}, \mathbb{V}_{n}^{\mathrm{an}}\right)$ given by $\epsilon_{J} \cdot\left(\left(z_{J}, z_{J_{F} \backslash J}\right), v\right)=\left(\left(-\bar{z}_{J}, z_{J_{F} \backslash J}\right), v\right)$ induces an action of the Weyl group on $\mathrm{H}^{d}\left(Y^{\mathrm{an}}, \mathbb{V}_{n}^{\mathrm{an}}\right)$ commuting with the Hecke action.

By Harder [19] we know that if $n \neq 0$ then $\mathrm{H}_{!}^{d}\left(Y^{\mathrm{an}}, \mathbb{V}_{n}(\mathbb{C})\right)=\mathrm{H}_{\text {cusp }}^{d}\left(Y^{\mathrm{an}}, \mathbb{V}_{n}(\mathbb{C})\right)$.

By (5) we have a Poincaré pairing $\langle\rangle:, \mathrm{H}^{d}\left(Y^{\mathrm{an}}, \mathbb{V}_{n}(R)\right) \times \mathrm{H}_{c}^{d}\left(Y^{\mathrm{an}}, \mathbb{V}_{n}(R)\right) \rightarrow R$.

Let $\eta$ be the idèle corresponding to the ideal $\mathfrak{n}$ and let $\iota=\left(\begin{array}{cc}0 & 1 \\ -\eta & 0\end{array}\right)$ be the Atkin-Lehner involution. By putting $[x, y]=\langle x, \iota y\rangle$ we obtain a new pairing

$$
[,]: \mathrm{H}_{!}^{d}\left(Y^{\mathrm{an}}, \mathbb{V}_{n}(R)\right) \times \mathrm{H}_{!}^{d}\left(Y^{\mathrm{an}}, \mathbb{V}_{n}(R)\right) \rightarrow R
$$

which is Hecke-equivariant. We call it the twisted Poincaré pairing.

Now we state the Eichler-Shimura-Harder isomorphism:

THEOREM 1.14 (Hida [25]). - If $n \neq 0$, then there exists an isomorphism:

$$
\delta: \bigoplus_{\psi} \bigoplus_{J \subset J_{F}} S_{k, J}(\mathfrak{n}, \psi) \cong \mathrm{H}_{!}^{d}\left(Y^{\mathrm{an}}, \mathbb{V}_{n}(\mathbb{C})\right)
$$

where $\psi$ runs over the Hecke characters of conductor dividing $\mathfrak{n}$ and type $-n_{0} t$ at infinity. This isomorphism is equivariant for the actions of the Hecke algebra and the Weyl group.

For each $J \subset J_{F}$ let $\hat{\epsilon}_{J}:\{ \pm 1\}^{J_{F}} \rightarrow\{ \pm 1\}$ be the unique character of the Weyl group sending $\epsilon_{\tau}=\left(-1_{\tau}, 1^{\tau}\right)$ to 1 , if $\tau \in J$, and to -1 if $\tau \in J_{F} \backslash J$. The restriction of the Eichler-ShimuraHarder isomorphism (7) to $S_{k, J}(\mathfrak{n}, \psi)$, followed by the projection on the $\left(\psi, \hat{\epsilon}_{J}\right)$-part yields a Hecke equivariant isomorphism

$$
\delta_{J}: S_{k, J}(\mathfrak{n}, \psi) \cong \mathrm{H}_{!}^{d}\left(Y, \mathbb{V}_{n}(\mathbb{C})\right)\left[\psi, \hat{\epsilon}_{J}\right] .
$$

Remark 1.15. - We have a direct sum decomposition:

$$
\mathrm{H}^{d}\left(M^{\mathrm{an}}, \mathbb{V}_{n}(\mathbb{C})\right)=\bigoplus_{J \subset J_{F}} \mathrm{H}^{d}\left(M^{\mathrm{an}}, \mathbb{V}_{n}(\mathbb{C})\right)\left[\widehat{\epsilon_{J} \otimes c}\right]
$$

where $c$ denotes the complex conjugation on the coefficients. This decomposition is finer than the usual Hodge decomposition, whose graded pieces are given by $(0 \leqslant a \leqslant d)$ :

$$
\operatorname{gr}^{a} \mathrm{H}^{d}\left(M^{\mathrm{an}}, \mathbb{V}_{n}(\mathbb{C})\right)=\bigoplus_{J \subset J_{F},|J|=a} \mathrm{H}^{d}\left(M^{\mathrm{an}}, \mathbb{V}_{n}(\mathbb{C})\right)\left[\widehat{\epsilon_{J} \otimes c}\right]
$$

$4^{\text {e }}$ SÉRIE - TOME $38-2005-\mathrm{N}^{\circ} 4$ 
The transcendental decomposition (9) has an algebraic interpretation, via the so-called BGG complex, that we will describe in the next section.

\section{Hodge-Tate weights of Hilbert modular varieties}

The aim of this section is to determine the Hodge-Tate weights of the $p$-adic étale cohomology of a Hilbert modular variety $\mathrm{H}^{\bullet}\left(M_{\overline{\mathbb{Q}}_{p}}, \mathbb{V}_{n}\left(\overline{\mathbb{Q}}_{p}\right)\right)$ as well as those of the $p$-adic Galois representation associated to a Hilbert modular form. In all this section we assume

(I) $p$ does not divide $\Delta=\mathrm{N}_{F / \mathbb{Q}}(\mathfrak{n} \mathfrak{d})$.

The proof relies on Faltings' Comparison Theorem [14] relating the étale cohomology of $M$ with coefficients in the local system $\mathbb{V}_{n}\left(\mathbb{Q}_{p}\right)$ to the de Rham logarithmic cohomology of the corresponding vector bundle $\overline{\mathcal{V}}_{n}$ over a smooth toroidal compactification $\bar{M}$ of $M$. The HodgeTate weights are given by the jumps of the Hodge filtration of the associated de Rham complex. These are computed, following [15], using the so-called Bernstein-Gelfand-Gelfand complex (BGG complex).

Instead of using Faltings' Comparison Theorem, one can apply Tsuji's results to the étale cohomology with constant coefficients of the Kuga-Sato variety $\mathcal{A}^{s}$ (s-fold fiber product of the universal Abelian variety $\mathcal{A}$ above the fine moduli space $M^{1}$ associated to $M$; see [11, Section 6] for the construction of toroidal compactifications of $\mathcal{A}^{s}$ ).

For each subset $J$ of $J_{F}$ we put $p(J)=\sum_{\tau \in J}\left(k_{0}-m_{\tau}-1\right) \tau+\sum_{\tau \in J_{F} \backslash J} m_{\tau} \tau \in \mathbb{Z}\left[J_{F}\right]$ and for each $a=\sum_{\tau \in J_{F}} a_{\tau} \tau \in \mathbb{Z}\left[J_{F}\right]$ we put $|a|=\sum_{\tau \in J_{F}} a_{\tau} \in \mathbb{Z}$.

\subsection{Motivic weight of the cohomology}

Consider the smooth sheaf $R^{1} \pi_{*} \overline{\mathbb{Q}}_{p}$ on $M^{1}$, where $\pi: \mathcal{A} \rightarrow M^{1}$ is the universal HBAV. It corresponds to a representation of the fundamental group of $M^{1}$ in $G\left(\overline{\mathbb{Q}}_{p}\right)$. By composing this representation with the algebraic representation $V_{n}$ of $G$ of highest weight $n$ (see Section 1.14), we obtain a smooth sheaf on $M^{1}$ (thus on $Y^{1}$ ). It descends to a smooth sheaf on $Y$, denoted by $\mathbb{V}_{n}\left(\overline{\mathbb{Q}}_{p}\right)$.

Let $W_{f}=\bigcap_{\mathfrak{a} \subset \mathfrak{o}} \operatorname{ker}\left(T_{\mathfrak{a}}-c(f, \mathfrak{a})\right)$ be the subspace of $\mathrm{H}^{d}\left(Y_{\overline{\mathbb{Q}}}, \mathbb{V}_{n}\left(\overline{\mathbb{Q}}_{p}\right)\right)$ corresponding to the Hilbert modular newform $f \in S_{k}(\mathfrak{n}, \psi)$. Put $s=\sum_{\tau}\left(n_{\tau}+2 m_{\tau}\right)=d n_{0}$.

PROPOSITION 2.1. $-W_{f}$ is pure of weight $d+s$, that is to say for all prime $l \nmid p \Delta$ the eigenvalues of the geometric Frobenius $\mathrm{Frob}_{l}$ at l are Weil numbers of absolute value $l^{\frac{d+s}{2}}$.

Proof. - Since $f$ is cuspidal $W_{f} \subset \mathrm{H}_{!}^{d}\left(Y_{\overline{\mathbb{Q}}}, \mathbb{V}_{n}\left(\overline{\mathbb{Q}}_{p}\right)\right)$. We recall that $Y_{\overline{\mathbb{Q}}}$ is a disjoint union of its connected components $M_{\overline{\mathbb{Q}}}=M_{1}\left(\mathfrak{c}_{i}, \mathfrak{n}\right)_{\overline{\mathbb{Q}}}$, where the $\mathfrak{c}_{i}$ 's form a set of representatives of $\mathrm{Cl}_{F}^{+}$. Let $\mathfrak{c}$ be one of the $\mathfrak{c}_{i}$ 's and $M^{1}=M_{1}^{1}(\mathfrak{c}, \mathfrak{n})$. For $*=\emptyset, c$ we have

$$
\mathrm{H}^{0}\left(\mathfrak{o}_{+}^{\times} / \mathfrak{o}_{\mathfrak{n}, 1}^{\times 2}, \mathrm{H}_{*}^{d}\left(M_{\overline{\mathbb{Q}}}^{1}, \mathbb{V}_{n}\left(\overline{\mathbb{Q}}_{p}\right)\right)\right)=\mathrm{H}_{*}^{d}\left(M_{\overline{\mathbb{Q}}}, \mathbb{V}_{n}\left(\overline{\mathbb{Q}}_{p}\right)\right)
$$

and therefore, it is enough to prove that $\mathrm{H}_{!}^{d}\left(M_{\overline{\mathbb{Q}}}, \mathbb{V}_{n}\left(\overline{\mathbb{Q}}_{p}\right)\right)$ is pure of weight $d+s$. We use Deligne's method [4]. Let $\pi: \mathcal{A} \rightarrow M^{1}$ be the universal Abelian variety (see Section 1.4). The sheaf $\mathbb{V}_{n}\left(\overline{\mathbb{Q}}_{p}\right)$ corresponds to the representation $\bigotimes_{\tau \in J_{F}} \operatorname{Sym}^{n_{\tau}} \otimes \operatorname{det}^{m_{\tau}}$ of the group $G^{*}$ and can therefore be cut out by algebraic correspondences in $\left(R^{1} \pi_{*} \overline{\mathbb{Q}}_{p}\right)^{\otimes s}$. Let $\pi_{s}: \mathcal{A}^{s} \rightarrow M^{1}$ be the Kuga-Sato variety. By the Kunneth's formula we have

$$
\mathrm{H}_{!}^{d}\left(M_{\overline{\mathbb{Q}}}^{1},\left(R^{1} \pi_{*} \overline{\mathbb{Q}}_{p}\right)^{\otimes s}\right) \subset \mathrm{H}_{!}^{d}\left(M_{\overline{\mathbb{Q}}}^{1}, R^{s} \pi_{s *} \overline{\mathbb{Q}}_{p}\right) \subset \mathrm{H}_{!}^{d+s}\left(\mathcal{A}_{\overline{\mathbb{Q}}}^{s}, \overline{\mathbb{Q}}_{p}\right) \subset \mathrm{H}^{d+s}\left(\overline{\mathcal{A}_{\overline{\mathbb{Q}}}^{s}}, \overline{\mathbb{Q}}_{p}\right),
$$


where the middle inclusion comes from the degeneration of the Leray spectral sequence $\mathrm{E}_{2 *}^{i, j}=\mathrm{H}_{*}^{i}\left(M_{\overline{\mathbb{Q}}}^{1}, R^{j} \pi_{s *} \overline{\mathbb{Q}}_{p}\right) \Rightarrow \mathrm{H}_{*}^{i+j}\left(\mathcal{A}_{\overline{\mathbb{Q}}}^{s}, \overline{\mathbb{Q}}_{p}\right)$ for $*=\emptyset, c$ (see [4]). The proposition is then a consequence of the Weil conjectures for the eigenvalues of the Frobenius, proved by Deligne [5].

\subsection{The Bernstein-Gelfand-Gelfand complex over $\overline{\mathbb{Q}}$}

In this and the next sections we describe, following Faltings [13], an algebraic construction of the transcendental decomposition of the Betti cohomology described in (9).

All the objects in this section are defined over a characteristic zero field splitting $G$.

Let $\mathfrak{g}, \mathfrak{b}, \mathfrak{t}$ and $\mathfrak{u}$ denote the Lie algebras of $G, B, T$ and $U$, respectively. Consider the canonical splitting $\mathfrak{g}=\mathfrak{b} \oplus \mathfrak{u}^{-}$. Let $U(\mathfrak{g}), U(\mathfrak{b})$ be the enveloping algebras of $\mathfrak{g}$ and $\mathfrak{b}$, respectively.

The aim of this section is to write down a resolution of $V_{n}$ of the type:

$$
0 \leftarrow V_{n} \leftarrow U(\mathfrak{g}) \otimes_{U(\mathfrak{b})} K_{n}^{\bullet},
$$

where the $K_{n}^{j}$ are finite-dimensional semi-simple $\mathfrak{b}$-modules, with explicit simple components.

We start by the case $n=0$. If we put $K_{0}^{j}=\bigwedge^{j}(\mathfrak{g} / \mathfrak{b})$ we obtain the so-called bar-resolution of $V_{0}$. Since $\bigwedge^{i}(\mathfrak{g} / \mathfrak{b})$ is a $\mathfrak{b}$-module with trivial $\mathfrak{u}$-action we deduce that $K_{0}^{j}=\bigoplus W_{\mu}$ with $\mu$ running over the weights of $B$ that are sums of $j$ distinct negative roots.

By tensoring this resolution with $V_{n}$ we obtain Koszul's complex:

$$
0 \leftarrow V_{n} \leftarrow U(\mathfrak{g}) \otimes_{U(\mathfrak{b})}\left(\left.\bigwedge^{j}(\mathfrak{g} / \mathfrak{b}) \otimes V_{n}\right|_{\mathfrak{b}}\right)
$$

which is a resolution of $V_{n}$ by $\mathfrak{b}$-modules $\left.\bigwedge^{i}(\mathfrak{g} / \mathfrak{b}) \otimes V_{n}\right|_{\mathfrak{b}}$, not semi-simple in general.

The BGG complex that we are going to define is a direct factor of Koszul's complex cut by the action of the center $U(\mathfrak{g})^{G}$ of $U(\mathfrak{g})$.

Denote by $\chi_{n}$ the weight $n$ character of $U(\mathfrak{g})^{G}$. It is a classical result that

LEMmA 2.2. $-\chi_{n}=\chi_{\mu}$ if, and only if, there exists $J \subset J_{F}$ such that $\mu=\epsilon_{J}(n+t)-t$.

By taking the $\chi_{n}$-part of the bar resolution (10) of $V_{n}$ we obtain a complex:

$$
0 \leftarrow V_{n} \leftarrow U(\mathfrak{g}) \otimes_{U(\mathfrak{b})} K_{n}^{\bullet}, \quad \text { with } K_{n}^{i}=\bigoplus_{J \subset J_{F},|J|=i} W_{\epsilon_{J}(n+t)-t},
$$

which is still a resolution of $V_{n}$, as a direct factor of a resolution. We call this resolution the BGG complex.

\subsection{Hodge-Tate decomposition of $\mathrm{H}^{\bullet}\left(M \otimes \overline{\mathbb{Q}}_{p}, \mathbb{V}_{n}\left(\overline{\mathbb{Q}}_{p}\right)\right)$}

In this paragraph we summarize the results of [11, Section 7]. The algebraic groups $G, B, T$ and $D$ of Section 1 have models over $\mathbb{Z}$, denoted by the same letters. For a scheme $S$, we put $S^{\prime}=S \times \operatorname{Spec}\left(\mathfrak{o}^{\prime}\left[\frac{1}{\Delta}\right]\right)$.

By Section 1.9 we can extend the vector bundles $\underline{\omega}$ and $\mathcal{H}_{\mathrm{dR}}^{1}$ to $\bar{M}^{\prime}$. Only the construction depends on a choice of a toroidal compactification $\bar{\pi}: \overline{\mathcal{A}} \rightarrow \overline{M^{1}}$ of $\pi: \mathcal{A} \rightarrow M^{1}$.

The sheaf $\underline{\mathfrak{M}}_{D}=\underline{\operatorname{Isom}}_{\mathfrak{o} \otimes \mathcal{O}_{\bar{M}}^{\prime}}\left(\underline{\omega}, \mathfrak{o} \otimes \mathcal{O}_{\bar{M}^{\prime}}\right)$ is a $D^{\prime}$-torsor over $\bar{M}^{\prime}$ (for the Zariski topology). We have a functor $\mathcal{F}_{D}$ from the category of algebraic representations of $D^{\prime}$ to the category of

$4^{\text {e }}$ SÉRIE - TOME $38-2005-\mathrm{N}^{\circ} 4$ 
vector bundles on $\bar{M}^{\prime}$ which are direct sums of invertible bundles. To an algebraic representation $W$ of $D^{\prime}, \mathcal{F}_{D}$ associates the fiber product $\overline{\mathcal{W}}:=\mathfrak{M}_{D} \stackrel{D^{\prime}}{\times} W$.

The sheaf $\underline{\mathfrak{M}_{B}}=\underline{\operatorname{Isom}}_{\mathfrak{o} \otimes \mathcal{O}_{\overline{M^{\prime}}}}^{\text {fil }}\left(\mathcal{H}_{\text {log-dR }}^{1},\left(\mathfrak{o} \otimes \mathcal{O}_{\bar{M}^{\prime}}\right)^{2}\right)$ is a $B^{\prime}$-torsor over $\bar{M}^{\prime}$. We have a functor $\mathcal{F}_{B}$ from the category of algebraic representations of $B^{\prime}$ to the category of filtered vector bundles on $\bar{M}^{\prime}$ whose graded are sums of invertible bundles. To an algebraic representation $V$ of $B^{\prime}, \mathcal{F}_{B}$ associates the fiber product $\overline{\mathcal{V}}:=\mathfrak{M}_{B} \stackrel{B^{\prime}}{\times} V$.

A representation of $G$ (respectively $T$ ) can be considered as a representation of $B$ by restriction (respectively by making $U$ act trivially). Thus, we may define a filtered vector bundle $\overline{\mathcal{V}}_{n}$ on $\bar{M}^{\prime}$ associated to the algebraic representation $V_{n}$ of $G$, and an invertible bundle $\overline{\mathcal{W}}_{n, n_{0}}$ on $\bar{M}^{\prime}$ associated to the algebraic representation of $T=D \times D$, given by $(u, \epsilon) \mapsto u^{n} \epsilon^{m}$.

The sheaf $\underline{\mathfrak{M}_{G}}=\underline{\operatorname{Isom}}_{\mathfrak{o} \otimes \mathcal{O}_{\overline{M^{\prime}}}}\left(\mathcal{H}_{\log -\mathrm{dR}}^{1},\left(\mathfrak{o} \otimes \mathcal{O}_{\overline{M^{\prime}}}\right)^{2}\right)$ is a $G^{\prime}$-torsor over $\bar{M}^{\prime}$. We have a functor $\mathcal{F}_{G}$ from the category of algebraic representations of $G^{\prime}$ to the category of flat vector bundles on $\bar{M}^{\prime}$ (that is vector bundles endowed with an integrable quasi-nilpotent logarithmic connection). To any algebraic representation $V$ of $B^{\prime}, \mathcal{F}_{G}$ associates the fiber product $\overline{\mathcal{V}}^{\nabla}:=\mathfrak{M}_{G} \stackrel{G^{\prime}}{\times} V$. For $j \in \mathbb{N}$, we put $\mathcal{H}_{\log \text {-dR }}^{j}\left(\bar{M}^{\prime}, \overline{\mathcal{V}}\right)=R^{j} \theta_{*}\left(\overline{\mathcal{V}}^{\nabla} \otimes \Omega_{\bar{M}^{\prime}}(\operatorname{dlog} \infty)\right)$, where $\theta: \bar{M}^{\prime} \rightarrow \operatorname{Spec}\left(\mathfrak{o}^{\prime}\left[\frac{1}{\Delta}\right]\right)$ denotes the structural homomorphism.

By Faltings' Comparison Theorem [14], the $\mathcal{G}_{\mathbb{Q}_{p}}$-representation $\mathrm{H}^{\bullet}\left(M_{\overline{\mathbb{Q}}_{p}}^{1}, \mathbb{V}_{n}\left(\overline{\mathbb{Q}}_{p}\right)\right)$ is crystalline, hence de Rham, and we have a canonical isomorphism

$$
\mathrm{H}^{\bullet}\left(M_{\overline{\mathbb{Q}}_{p}}^{1}, \mathbb{V}_{n}\left(\overline{\mathbb{Q}}_{p}\right)\right) \otimes B_{\mathrm{dR}} \cong \mathcal{H}_{\mathrm{log}-\mathrm{dR}}\left(\bar{M}^{1} / \overline{\mathbb{Q}}_{p}, \overline{\mathcal{V}}_{n}\right) \otimes B_{\mathrm{dR}}
$$

By [11, Section 7] the Hodge to de Rham spectral sequence

$$
\mathrm{E}_{1}^{i, j}=\mathrm{H}^{i+j}\left({\overline{M^{1}}}_{/ \overline{\mathbb{Q}}_{p}}, \operatorname{gr}^{i}\left(\overline{\mathcal{V}}_{n} \otimes \Omega_{\bar{M}^{1}}^{\bullet}(\operatorname{dlog} \infty)\right)\right) \Longrightarrow \mathcal{H}_{\log -\mathrm{dR}}^{i+j}\left({\overline{M^{1}}}_{/ \overline{\mathbb{Q}}_{p}}, \overline{\mathcal{V}}_{n}\right),
$$

degenerates at $\mathrm{E}_{1}$ (the filtration being the tensor product of the two Hodge filtrations). In order to compute the jumps of the resulting filtration we introduce the BGG complex:

$$
\overline{\mathcal{K}}_{n}^{i}=\bigoplus_{J \subset J_{F},|J|=i} \overline{\mathcal{W}}_{\epsilon_{J}(n+t)-t, n_{0}} .
$$

The fact that $\overline{\mathcal{K}}_{n}^{\bullet}$ is a complex follows from (11) and from the following isomorphism (see [15, Proposition VI.5.1])

$$
\operatorname{Hom}_{U(\mathfrak{g})}\left(U(\mathfrak{g}) \otimes_{U(\mathfrak{b})} W_{1}\right),\left(U(\mathfrak{g}) \otimes_{U(\mathfrak{b})} W_{2}\right) \rightarrow \operatorname{Diff.Op} .\left(\overline{\mathcal{W}}_{2}, \overline{\mathcal{W}}_{1}\right)
$$

Define a filtration on $\overline{\mathcal{K}}_{n}^{\bullet}$ by $\mathrm{Fil}^{i} \overline{\mathcal{K}}_{n}^{\bullet}=\bigoplus_{J \subset J_{F},|p(J)| \geqslant i} \overline{\mathcal{W}}_{\epsilon_{J}(n+t)-t, n_{0}}$.

The image of Koszul's complex (10) by the contravariant functor $W \mapsto \overline{\mathcal{W}}$ is equal to the de Rham complex. Since the BGG complex is a direct (filtered) factor of the Koszul's complex, we obtain:

THEOREM 2.3 [11, Theorem 7.8]. -

(i) There is a quasi-isomorphism of filtered complexes

$$
\overline{\mathcal{K}}_{n}^{\bullet} \hookrightarrow \overline{\mathcal{V}}_{n} \otimes \Omega_{\overline{M^{1}}}^{\bullet}(\operatorname{dlog} \infty) .
$$


(ii) The spectral sequence given by the Hodge filtration

$$
\mathrm{E}_{1}^{i, j}=\bigoplus_{J \subset J_{F},|p(J)|=i} \mathrm{H}^{i+j-|J|}\left(\bar{M}_{/ \overline{\mathbb{Q}}_{p}}, \overline{\mathcal{W}}_{\epsilon_{J}(n+t)-t, n_{0}}\right) \Longrightarrow \mathcal{H}_{\log -\mathrm{dR}}^{i+j}\left(\bar{M}_{/ \overline{\mathbb{Q}}_{p}}, \overline{\mathcal{V}}_{n}\right)
$$

degenerates at $\mathrm{E}_{1}$.

(iii) For all $j \leqslant d$, the Hodge-Tate weights of the p-adic representation $\mathrm{H}^{j}\left(M_{\overline{\mathbb{Q}}_{p}}^{1}, \mathbb{V}_{n}\left(\overline{\mathbb{Q}}_{p}\right)\right)$ belong to the set $\{|p(J)|,|J| \leqslant j\}$.

\subsection{Hecke operators on the cohomology}

We describe the standard Hecke operator $T_{\mathfrak{a}}$ as a correspondence on $Y^{1}$. We are indebted to M. Kisin for pointing us out that the usual definition of Hecke operators on $Y$ extends to $Y^{1}$ (see $[29, \S 1.9-1.11])$. Note that the corresponding Hecke action on analytic modular forms for $G^{*}$ (see Section 1.10) is not easy to write down, because the double class for the Hecke operator $T_{v}$ does not belong to $G^{*}\left(\mathbb{A}_{f}\right)$, unless $v$ is inert in $F$.

Recall that $Y_{1}^{1}(\mathfrak{n})=\coprod_{i=1}^{h_{F}^{+}} M_{1}^{1}\left(\mathfrak{c}_{i}, \mathfrak{n}\right)$, where $\mathfrak{c}_{1}, \ldots, \mathfrak{c}_{h_{F}^{+}}$form a set of representatives of $\mathrm{Cl}_{F}^{+}$.

Assume that $\mathfrak{c}_{i} \mathfrak{a}$ and $\mathfrak{c}_{j}$ have the same class in $\mathrm{Cl}_{F}^{+}$. Consider the contravariant functor $\underline{\mathcal{M}}_{\mathfrak{a}}^{1}$ from the category of $\mathbb{Z}\left[\frac{1}{\Delta}\right]$-schemes to the category of sets, assigning to a scheme $S$ the set of isomorphism classes of quintuples $(A, \lambda, \alpha, C, \beta)$ where $(A, \lambda, \alpha) / S$ is a $\mathfrak{c}_{i}$-polarized HBAV with $\mu_{\mathfrak{n}}$-level structure, $C$ is a closed subscheme of $A[\mathfrak{a}]$ which is $\mathfrak{o}$-stable, disjoint from $\alpha\left(\mathbb{G}_{m} \otimes \mathfrak{d}^{-1}\right)$ and locally isomorphic to the constant group scheme $\mathfrak{o} / \mathfrak{a}$ over $S$, and $\beta$ is an $\mathfrak{o}_{\mathfrak{n}, 1}^{\times 2}$-orbit of isomorphisms $\left(\mathfrak{c}_{i} \mathfrak{a},\left(\mathfrak{c}_{i} \mathfrak{a}\right)_{+}\right) \stackrel{\sim}{\longrightarrow}\left(\mathfrak{c}_{j}, \mathfrak{c}_{j+}\right)$.

We have a projection $\mathcal{M}_{\mathfrak{a}}^{1} \rightarrow \mathcal{M}^{1},(A, \lambda, \alpha, C, \beta) \mapsto(A, \lambda, \alpha)$ which is relatively representable by $\pi_{1}: M_{\mathfrak{a}}^{1}\left(\mathfrak{c}_{i}, \mathfrak{n}\right) \rightarrow M_{1}^{1}\left(\mathfrak{c}_{i}, \mathfrak{n}\right)$. We have also a projection $\pi_{2}: M_{\mathfrak{a}}^{1}\left(\mathfrak{c}_{i}, \mathfrak{n}\right) \rightarrow M_{1}^{1}\left(\mathfrak{c}_{j}, \mathfrak{n}\right)$ coming from $(A, \lambda, \alpha, C, \beta) \mapsto\left(A / C, \lambda^{\prime}, \alpha^{\prime}\right)$, where $\alpha^{\prime}$ is the composed map of $\alpha$ and $A \rightarrow A / C$ and $\lambda^{\prime}$ is a $\mathfrak{c}_{j}$-polarization of $A / C$ (defined via $\lambda$ and $\beta$ ).

Put $Y_{\mathfrak{a}}^{1}=\coprod_{i=1}^{h_{F}^{+}} M_{\mathfrak{a}}^{1}\left(\mathfrak{c}_{i}, \mathfrak{n}\right)$. As $\mathfrak{c}_{i} \mapsto \mathfrak{c}_{j} \simeq \mathfrak{c}_{i} \mathfrak{a}$ is a permutation of $\mathrm{Cl}_{F}^{+}$, we get two finite projections $\pi_{1}, \pi_{2}: Y_{\mathfrak{a}}^{1} \rightarrow Y^{1}$ :

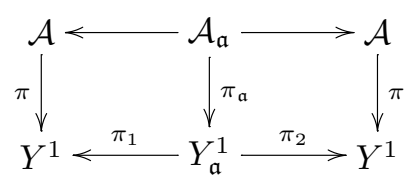

From this diagram we obtain $\pi_{2}^{*} \mathcal{H}_{\mathrm{dR}}^{1} \rightarrow \pi_{1}^{*} \mathcal{H}_{\mathrm{dR}}^{1}$. Therefore, for every algebraic representation $V$ of $G$, we have $\pi_{2}^{*} \mathcal{V}^{\nabla} \rightarrow \pi_{1}^{*} \mathcal{V}^{\nabla}$. By composing this morphism by $\pi_{1 *}$ and taking the trace, we obtain $\mathcal{V}^{\nabla} \rightarrow \pi_{1 *} \pi_{2}^{*} \mathcal{V}^{\nabla} \rightarrow \pi_{1 *} \pi_{1}^{*} \mathcal{V}^{\nabla} \rightarrow \mathcal{V}^{\nabla}$. This gives an action of $T_{\mathfrak{a}}$ on $\mathrm{H}^{\bullet}\left(Y^{1}, \mathcal{V}^{\nabla}\right)$.

The same way, the above diagram gives $\pi_{2}^{*} \underline{\omega} \rightarrow \pi_{1}^{*} \underline{\omega}$ and $\pi_{2}^{*} \underline{\nu} \rightarrow \pi_{1}^{*} \underline{\nu}$. Therefore, for each algebraic representation $W$ of $T$, we get $\pi_{2}^{*} \mathcal{W} \rightarrow \pi_{1}^{*} \mathcal{W}$. In order to define the action of $T_{\mathfrak{a}}$ on Hilbert modular forms, we need to modify the last arrow: we decompose $\mathcal{W}$ as $\left(\mathcal{W} \otimes \underline{\omega}^{-2 t}\right) \otimes \underline{\omega}^{2 t}$ and we define $\pi_{2 *}\left(\mathcal{W} \underline{\omega}^{-2 t}\right) \rightarrow \pi_{1 *}\left(\mathcal{W} \underline{\omega}^{-2 t}\right)$ as above and $\pi_{2 *} \underline{\omega}^{2 t} \rightarrow \pi_{1 * \underline{\omega}^{2 t}}$ via the KodairaSpencer isomorphism $\Omega_{Y^{1}}^{1} \simeq \underline{\omega}^{2} \otimes_{\mathfrak{o}} \mathfrak{d c}^{-1}$ as in [29, §1.11]. Thus we obtain $\mathcal{W} \rightarrow \pi_{1 *} \pi_{2}^{*} \mathcal{W} \rightarrow$ $\pi_{1 *} \pi_{1}^{*} \mathcal{W} \rightarrow \mathcal{W}$ and an action of $T_{\mathfrak{a}}$ on $\mathrm{H}^{\bullet}\left(Y^{1}, \mathcal{W}\right)$.

In particular, we obtain an action of $T_{\mathfrak{a}}$ on the space $\mathrm{H}^{0}\left(Y^{1}, \underline{\omega}^{k} \otimes \underline{\nu}^{-n_{0} t / 2}\right)$ of geometric Hilbert modular forms for $G^{*}$. As it has been observed in $[29,1.11 .8]$ over $\mathbb{C}$ this action is given 
by the projection

$$
\frac{1}{\left[\mathfrak{o}_{+}^{\times}: \mathfrak{o}_{\mathfrak{n}, 1}^{\times}\right]} \sum_{[\epsilon] \in \mathfrak{o}_{+}^{\times} / \mathfrak{o}_{\mathfrak{n}, 1}^{\times}}[\epsilon] \cdot: \mathrm{H}^{0}\left(Y^{1}, \underline{\omega}^{k} \otimes \underline{\nu}^{-n_{0} t / 2}\right) \rightarrow \mathrm{H}^{0}\left(Y, \underline{\omega}^{k} \otimes \underline{\nu}^{-n_{0} t / 2}\right),
$$

followed by the usual Hecke operator on the space of Hilbert modular forms (see Section 1.10).

\subsection{Hodge-Tate weights of $\otimes \operatorname{Ind}_{F}^{\mathbb{Q}} \rho$ in the crystalline case}

We first recall the notion of induced representation. Let $V_{0}$ be a vector space over a field $L$, and let $\rho_{0}: \mathcal{G}_{F} \rightarrow \mathrm{GL}\left(V_{0}\right)$ be a linear representation. The induced representation $\operatorname{Ind}_{F}^{\mathbb{Q}} \rho_{0}$ of $\rho_{0}$ from $F$ to $\mathbb{Q}$ is by definition the $L$-vector space

$$
\operatorname{Hom}_{\mathcal{G}_{F}}\left(\mathcal{G}_{\mathbb{Q}}, V_{0}\right):=\left\{\phi_{0}: \mathcal{G}_{\mathbb{Q}} \rightarrow V_{0} \mid \forall x \in \mathcal{G}_{F}, y \in \mathcal{G}_{\mathbb{Q}}, \phi_{0}(y x)=\rho_{0}\left(x^{-1}\right)\left(\phi_{0}(y)\right)\right\},
$$

where $y \in \mathcal{G}_{\mathbb{Q}}$ acts on $\phi_{0} \in \operatorname{Hom}_{\mathcal{G}_{F}}\left(\mathcal{G}_{\mathbb{Q}}, V_{0}\right)$ by $y \cdot \phi_{0}(\cdot)=\phi_{0}\left(y^{-1} \cdot\right)$.

For any fixed decomposition $\mathcal{G}_{\mathbb{Q}}=\coprod_{\tau \in J_{F}} \tilde{\tau} \mathcal{G}_{F}$, the map $\phi_{0} \mapsto\left(\phi_{0}(\tilde{\tau})\right)_{\tau}$ gives an isomorphism between $\operatorname{Hom}_{\mathcal{G}_{F}}\left(\mathcal{G}_{\mathbb{Q}}, V_{0}\right)$ and the direct sum $\bigoplus_{\tau} V_{\tau}$ (where each $V_{\tau}$ is isomorphic to $V_{0}$ ). Via this identification, the action of $\mathcal{G}_{\mathbb{Q}}$ on $\bigoplus_{\tau} V_{\tau}$ is given by:

$$
\left(\operatorname{Ind}_{F}^{\mathbb{Q}} \rho_{0}\right)(y)\left(\left(v_{\tau}\right)_{\tau}\right)=\left(\rho_{0}\left(\tilde{\tau}^{-1} y \tilde{\tau}_{y}\right)\left(v_{\tau_{y}}\right)\right)_{\tau},
$$

where $y^{-1} \tilde{\tau} \in \tilde{\tau}_{y} \mathcal{G}_{F}$. In fact $\left(\phi_{0}(\tilde{\tau})\right)_{\tau} \stackrel{y}{\mapsto}\left(\phi_{0}\left(y^{-1} \tilde{\tau}\right)\right)_{\tau}=\left(\rho_{0}\left(\tilde{\tau}^{-1} y \tilde{\tau}_{y}\right)\left(\phi_{0}\left(\tilde{\tau}_{y}\right)\right)\right)_{\tau}$.

Keeping the same notations we define, following Yoshida [44], the tensor induced representation $\otimes \operatorname{Ind}_{F}^{\mathbb{Q}} \rho_{0}: \mathcal{G}_{\mathbb{Q}} \rightarrow \mathrm{GL}\left(\otimes_{\tau} V_{\tau}\right)$ as:

$$
\left(\bigotimes \operatorname{Ind}_{F}^{\mathbb{Q}} \rho_{0}\right)(y)\left(\bigotimes_{\tau} v_{\tau}\right)=\bigotimes_{\tau} \rho_{0}\left(\tilde{\tau}^{-1} y \tilde{\tau}_{y}\right)\left(v_{\tau_{y}}\right) .
$$

Remark 2.4. - For each $y \in \mathcal{G}_{\mathbb{Q}}$ the map $\tau \mapsto \tau_{y}$ is a permutation of $J_{F}$ and it is trivial if, and only if, $y \in \mathcal{G}_{\widetilde{F}}$. Therefore, for each $y \in \mathcal{G}_{\widetilde{F}}$, we have $\left(\otimes \operatorname{Ind}_{F}^{\mathbb{Q}} \rho_{0}\right)(y)=\bigotimes_{\tau} \rho_{0}\left(\tilde{\tau}^{-1} y \tilde{\tau}\right)$. Moreover for all $y, y^{\prime} \in \mathcal{G}_{\mathbb{Q}}$ we have $\left(\tau_{y}\right)_{y^{\prime}}=\tau_{y y^{\prime}}$.

Definition 2.5. - The internal conjugate $g_{\tau}$ of $g \in S_{k, J}(\mathfrak{n}, \psi)$ by $\tau \in J_{F}$ is defined as the unique element $g_{\tau} \in S_{k^{\tau}, J^{\tau}}\left(\tau(\mathfrak{n}), \psi_{\tau}\right)$ satisfying $c\left(g_{\tau}, \mathfrak{a}\right)=c(g, \tau(\mathfrak{a}))$ for each ideal $\mathfrak{a}$ of $\mathfrak{o}$, where $k^{\tau}=\sum_{\tau^{\prime}} k_{\tau \tau^{\prime}} \tau^{\prime}$ and $\psi_{\tau}(\mathfrak{a})=\psi(\tau(\mathfrak{a}))$. It is a Hilbert modular form on $\tau(F)$.

If $\rho=\rho_{f, p}$ by the previous remark we have $\left(\bigotimes \operatorname{Ind}_{F}^{\mathbb{Q}} \rho\right)(y)=\bigotimes_{\tau} \rho_{f_{\tau}}(y)$ for all $y \in \mathcal{G}_{\widetilde{F}}$.

Brylinski and Labesse [3] have shown (see [40] for this formulation):

THEOREM 2.6 (Brylinski-Labesse). - The restrictions to $\mathcal{G}_{\widetilde{F}}$ of the two $\mathcal{G}_{\mathbb{Q}}$-representations $W_{f}$ and $\otimes \operatorname{Ind}_{F}^{\mathbb{Q}} \rho$ have the same characteristic polynomial.

COROLlaRY 2.7 [11, Corollary 7.9].-

(i) The spectral sequence given by the Hodge filtration

$$
\mathrm{E}_{1}^{i, j}=\bigoplus_{J \subset J_{F},|p(J)|=i} \mathrm{H}^{i+j-|J|}\left(\bar{M}_{/ \overline{\mathbb{Q}}_{p}}, \overline{\mathcal{W}}_{\epsilon_{J}(n+t)-t, n_{0}}\right) \Longrightarrow \mathcal{H}_{\log -\mathrm{dR}}^{i+j}\left(\bar{M}_{/ \overline{\mathbb{Q}}_{p}}, \overline{\mathcal{V}}_{n}\right)
$$

degenerates at $\mathrm{E}_{1}$ and is Hecke equivariant.

(ii) The Hodge-Tate weights of $W_{f}$ are given by the multiset $\left\{|p(J)|, J \subset J_{F}\right\}$. 
Proof. - (i) By taking the invariants of the Hodge filtration of $\overline{\mathcal{V}}_{n} \otimes \Omega_{M^{1}}^{\bullet}($ dlog $\infty)$ by the Galois group of the étale covering $\overline{M^{1}} \rightarrow \bar{M}$ we obtain a filtration of the complex $\overline{\mathcal{V}}_{n} \otimes$ $\Omega_{\bar{M}}(\operatorname{dlog} \infty)$ on $\bar{M}^{\prime}$, still called the Hodge filtration. The same way, we define the BGG complex over $\bar{M}^{\prime}$ by taking the invariants of the BGG complex over $\overline{M^{1}}$. The associated spectral sequence is given by the invariants of the spectral sequence of Theorem 2.3(ii). We have now to see that it is Hecke equivariant.

The Hecke operator $T_{\mathfrak{a}}$ extends to a correspondence on $\overline{Y^{1}}$. One way to define it is to take the schematic closure of $T_{\mathfrak{a}} \subset Y^{1} \times Y^{1}$ in $\overline{Y^{1}} \times \overline{Y^{1}}$. Another way is to take a toroidal compactification $\overline{Y_{\mathfrak{a}}^{1}}$ of $Y_{\mathfrak{a}}^{1}$ over the toroidal compactification $\overline{Y^{1}}$ of $Y^{1}$.

Hence $T_{\mathfrak{a}}$ acts on $\mathrm{H}^{\bullet}\left(\overline{Y^{1}}, \overline{\mathcal{W}}\right)$ and on $\mathrm{H}^{\bullet}\left(\overline{Y^{1}}, \overline{\mathcal{V}}^{\nabla}\right)$. Moreover, the $T_{\mathfrak{a}}$ 's commute. In fact they commute on the right-hand side of Theorem 2.3(ii), because this side is independent of the toroidal compactification by Faltings' Comparison Theorem. Since the spectral sequence of Theorem 2.3(ii) degenerates at $\mathrm{E}_{1}$, they also commute on the left-hand side.

(ii) We have $\overline{\mathcal{W}}_{\epsilon_{J}(n+t)-t, n_{0}}=\underline{\omega}^{-\epsilon_{J}(n+t)+t} \otimes \underline{\nu}^{p(J)}$. It follows from Theorem 2.3 (as in [15, Theorem 5.5] and [31, Section 2.3]) that the jumps of the Hodge filtration are among $|p(J)|$, $J \subset J_{F}$.

Moreover gr ${ }^{|p(J)|} \mathrm{H}^{d}\left(\bar{M}_{/ \overline{\mathbb{Q}}_{p}}, \overline{\mathcal{V}}_{n} \otimes \Omega_{\bar{M}^{\bullet}}(\operatorname{dlog} \infty)\right)=\mathrm{H}^{d-|J|}\left(\bar{M}_{/ \overline{\mathbb{Q}}_{p}}, \underline{\omega}^{-\epsilon_{J}(n+t)+t} \otimes \underline{\nu}^{p(J)}\right)$.

It is enough to see that the $\overline{\mathbb{Q}}_{p}$-vector space $\mathrm{H}^{d-|J|}\left(\bar{Y}_{\overline{\mathbb{Q}}_{p}}, \underline{\omega}^{-\epsilon_{J}(n+t)+t} \otimes \underline{\nu}^{p(J)}\right)[f]$ is of dimension 1 for all $J \subset J_{F}$.

By the existence of a BGG complex over $\overline{\mathbb{Q}}$ giving by base change the BGG complexes over $\overline{\mathbb{Q}}_{p}$ and $\mathbb{C}$, we have a Hecke-equivariant isomorphism

$$
\mathrm{H}^{d-|J|}\left(\bar{Y}_{\overline{\mathbb{Q}}_{p}}, \underline{\omega}^{-\epsilon_{J}(n+t)+t} \otimes \underline{\nu}^{p(J)}\right) \otimes_{\overline{\mathbb{Q}}_{p}} \mathbb{C}=\mathrm{H}^{d}\left(Y^{\mathrm{an}}, \mathbb{V}_{n}(\mathbb{C})\right)\left[\widehat{\epsilon_{J} \otimes c}\right]
$$

For all $J \subset J_{F}$, the $f$-part of $\mathrm{H}^{d}\left(Y^{\text {an }}, \mathbb{V}_{n}(\mathbb{C})\right)\left[\widehat{\epsilon_{J} \otimes c}\right]$ is equal to $\mathrm{H}_{!}^{d}\left(Y^{\text {an }}, \mathbb{V}_{n}(\mathbb{C})\right)\left[\widehat{\epsilon_{J} \otimes c}, f\right]$ and is therefore one dimensional by (8).

\section{Remark 2.8. -}

(1) We proved that $W_{f}$ is pure of weight $d\left(k_{0}-1\right)$. The set of its Hodge-Tate weights is stable by the symmetry $h \mapsto d\left(k_{0}-1\right)-h$, since $\left|p\left(J_{F} \backslash J\right)\right|=d\left(k_{0}-1\right)-|p(J)|$. This symmetry is induced by the Poincaré duality $W_{f} \times W_{f} \rightarrow \overline{\mathbb{Q}}_{p}\left(-d\left(k_{0}-1\right)\right)$.

(2) If $F$ is a real quadratic field and $\tau$ denotes the non-trivial automorphism of $F$, then the Hodge-Tate weights of $W_{f}$ are given by $m_{\tau}, k_{0}-m_{\tau}-1, k_{0}+m_{\tau}-1,2 k_{0}-m_{\tau}-2$.

\subsection{Hodge-Tate weights of $\rho$ in the crystalline case}

The embedding $\iota_{p}: \overline{\mathbb{Q}} \hookrightarrow \overline{\mathbb{Q}}_{p}$ allows us to identify $J_{F}$ with $\operatorname{Hom}_{\mathbb{Q}-\text { alg. }}\left(F, \overline{\mathbb{Q}}_{p}\right)$. For each prime $\mathfrak{p}$ of $F$ dividing $p$, we put $J_{F, \mathfrak{p}}=\operatorname{Hom}_{\mathbb{Q}_{p}-\text { alg. }}\left(F_{\mathfrak{p}}, \overline{\mathbb{Q}}_{p}\right)$. Thus we get a partition $J_{F}=\coprod_{\mathfrak{p}} J_{F, \mathfrak{p}}$. Let $D_{\mathfrak{p}}$ (respectively $I_{\mathfrak{p}}$ ) be a decomposition (respectively inertia) subgroup of $\mathcal{G}_{F}$ at $\mathfrak{p}$.

The following result is due to Wiles if $k$ is parallel, and to Hida in the general case.

THEOREM 2.9 (Wiles [43], Hida [23]). - Assume that $f$ is ordinary at $p$ (see Definition 1.13). Then $\rho_{\mid D_{\mathfrak{p}}}$ is reducible and:

(ORD)

$$
\rho_{\mid I_{\mathfrak{p}}} \sim\left(\begin{array}{cc}
\tilde{\varepsilon}_{\mathfrak{p}} & * \\
0 & \tilde{\delta}_{\mathfrak{p}}
\end{array}\right)
$$

where $\tilde{\delta}_{\mathfrak{p}}$ (respectively $\tilde{\varepsilon}_{\mathfrak{p}}$ ) is obtained by composing the class field theory map $I_{\mathfrak{p}} \rightarrow \mathfrak{o}_{\mathfrak{p}}^{\times}$with the map $\mathfrak{o}_{\mathfrak{p}}^{\times} \rightarrow \overline{\mathbb{Q}}_{p}^{\times}, x \mapsto \prod_{\tau \in J_{F, \mathfrak{p}}} \tau(x)^{-m_{\tau}}$ (respectively $x \mapsto \prod_{\tau \in J_{F, \mathfrak{p}}} \tau(x)^{-\left(k_{0}-m_{\tau}-1\right)}$ ). 
Breuil [2] has shown that if $p>k_{0}$ and $p$ does not divide $\Delta$, then $\rho$ is crystalline at each prime $\mathfrak{p}$ of $F$ dividing $p$, with Hodge-Tate weights between 0 and $k_{0}-1$.

COROllary 2.10. - Assume $p>k_{0}$ and that $p$ does not divide $\Delta$. Then for each prime $\mathfrak{p}$ of $F$ dividing $p, \rho_{\mid D_{\mathfrak{p}}}$ is crystalline with Hodge-Tate weights the $2\left[F_{\mathfrak{p}}: \mathbb{Q}_{p}\right]$ integers $\left(m_{\tau}\right.$, $\left.k_{0}-m_{\tau}-1\right)_{\tau \in J_{F, \mathfrak{p}}}$.

Proof. - Assume first that $n \neq 0$. Let $K$ be a CM quadratic extension of $F$, splitting all the primes $\mathfrak{p}$ of $F$ dividing $p$. Blasius and Rogawski [1] have constructed a pure motive over $K$ with Hodge weights $\left(m_{\tau}, k_{0}-m_{\tau}-1\right)_{\tau \in J_{F}}$, whose $p$-adic realization is isomorphic to the restriction of $\rho$ to $\mathcal{G}_{K}$. This shows that $\rho_{\mid D_{\mathfrak{p}}}$ is de Rham for all $p$, and crystalline for $p$ big enough.

By Faltings' Comparison Theorem the Hodge weights of this motive correspond via $\iota_{p}: \overline{\mathbb{Q}} \hookrightarrow \overline{\mathbb{Q}}_{p}$ to the Hodge-Tate weights of its $p$-adic realization, which are the same as the Hodge-Tate weights of $\rho$ at primes $\mathfrak{p}$ dividing $p$. This proves the corollary for $n \neq 0$.

If $n=0$ (or more generally if $k$ is parallel) we can complete the proof using the following

LEMMA 2.11. - Let $a$ and $b$ be two positive integers and let $\left(a_{\tau}\right)_{\tau \in J_{F}}$ (respectively $\left(b_{\tau}\right)_{\tau \in J_{F}}$ ) be integers satisfying $0 \leqslant 2 a_{\tau}<a$ (respectively $0 \leqslant 2 b_{\tau}<b$ ). Assume that the following two multisets are equal

$$
\left\{\sum_{\tau \in J} a_{\tau}+\sum_{\tau \in J_{F} \backslash J}\left(a-a_{\tau}\right), J \subset J_{F}\right\}=\left\{\sum_{\tau \in J} b_{\tau}+\sum_{\tau \in J_{F} \backslash J}\left(b-b_{\tau}\right), J \subset J_{F}\right\} .
$$

Then $a=b$ and we have equality of multisets $\left\{a_{\tau}, \tau \in J_{F}\right\}=\left\{b_{\tau}, \tau \in J_{F}\right\}$.

Using this lemma together with Theorem 2.6 and Corollary 2.7(ii) we obtain, up to permutation, the Hodge-Tate weights of $\rho$ at primes $\mathfrak{p}$ dividing $p$. In particular, we know exactly the Hodge-Tate weights of $\rho$ when $k$ is parallel.

\subsection{Fontaine-Laffaille weights of $\bar{\rho}$ in the crystalline case}

Our aim is to find the weights of $\bar{\rho}_{\mid I_{\mathfrak{p}}}$ for $\mathfrak{p}$ dividing $p$. If $f$ is ordinary at $p$ we know by Theorem 2.9 that $\rho_{\mid D_{\mathrm{p}}}$ is reducible and by a simple reduction modulo $\mathcal{P}$ we obtain the weights of $\bar{\rho}_{\mid I_{\mathfrak{p}}}$.

Proposition 2.12. - Assume $p>k_{0}$ and that $p$ does not divide $\Delta$. Then $\bar{\rho}$ is crystalline at each $\mathfrak{p}$ dividing $p$ with Fontaine-Laffaille weights $\left(m_{\tau}, k_{0}-m_{\tau}-1\right)_{\tau \in J_{F, \mathfrak{p}}}$.

Proof. - It follows from Fontaine-Laffaille's theory [17] and from the computation of HodgeTate weights of $\rho_{\mid D_{\mathfrak{p}}}$ from Section 2.6.

Consider a Galois stable lattice $\mathcal{O}^{2}$ in the crystalline representation $\rho$, as well as the sublattice $\mathcal{P}^{2}$. The representation $\bar{\rho}$ is equal to the quotient of these two lattices. It is crystalline, as a sub-quotient of a crystalline representation. Its weights are determined by the associated filtered Fontaine-Laffaille module. Since the Fontaine-Laffaille functor is exact, this module is given by the quotient of Fontaine-Laffaille's filtered modules associated to the two lattices. By compatibility of the filtrations on these two lattices, and by the condition $p>k_{0}$, the graded of the quotient have the right dimension.

COROLLARY 2.13. - Let $\mathfrak{p}$ be a prime of $F$ above $p$. Then

$$
\bar{\rho}_{\mid I_{\mathfrak{p}}} \sim\left(\begin{array}{cc}
\varepsilon_{\mathfrak{p}} & * \\
0 & \delta_{\mathfrak{p}}
\end{array}\right),
$$


where $\varepsilon_{\mathfrak{p}}, \delta_{\mathfrak{p}}: I_{\mathfrak{p}} \rightarrow \overline{\mathbb{F}}_{p}^{\times}$are two tame characters of level $\left|J_{F, \mathfrak{p}}\right|$ or $2\left|J_{F, \mathfrak{p}}\right|$, whose product equals the $\left(1-k_{0}\right)$ th power of the modulo p cyclotomic character and whose sum has Fontaine-Laffaille weights $\left(m_{\tau}, k_{0}-m_{\tau}-1\right)_{\tau \in J_{F, p}}$.

\section{Study of the images of $\bar{\rho}$ and $\operatorname{Ind}_{F}^{\mathbb{Q}} \bar{\rho}$}

In all this section we assume that $p>k_{0}$ and $p$ does not divide $6 \Delta$.

Let $\omega: \mathcal{G}_{\mathbb{Q}} \rightarrow \mathbb{F}_{p}^{\times}$be the modulo $p$ cyclotomic character and let pr: $\mathrm{GL}_{2}(\kappa) \rightarrow \mathrm{PGL}_{2}(\kappa)$ be the canonical projection. We recall that $\bar{\rho}=\bar{\rho}_{f, p}$.

\subsection{Lifting of characters and irreducibility criterion for $\bar{\rho}$}

PROPOSITION 3.1.-

(i) For all but finitely many primes $p\left(\mathbf{I r r}_{\bar{\rho}}\right)$ holds, that is $\bar{\rho}$ is absolutely irreducible.

(ii) Assume that $k$ is non-parallel. If for all $J \subset J_{F}$ there exists $\epsilon \in \mathfrak{o}_{+}^{\times}, \epsilon-1 \in \mathfrak{n}$, such that $p$ does not divide the non-zero integer $\mathrm{N}_{F / \mathbb{Q}}\left(\epsilon^{p(J)}-1\right)$, then $\left(\mathbf{I r r}_{\bar{\rho}}\right)$ holds.

Remark 3.2. - Assume that $k=k_{0} t$ is parallel and that for all $\emptyset \subsetneq J \subsetneq J_{F}$, there exists $\epsilon \in \mathfrak{o}_{+}^{\times}$, $\epsilon-1 \in \mathfrak{n}$ such that $p$ does not divide the non-zero integer $\mathrm{N}_{F / \mathbb{Q}}\left(\epsilon^{p(J)}-1\right)$. Then we expect $\bar{\rho}$ to be absolutely irreducible, unless $p$ divides the constant term of an Eisenstein series of weight $k$ and level dividing $\mathfrak{n}$, that is the numerator of the value at $1-k_{0}$ of the $L$-function of a finite order Hecke character of $F$ of conductor dividing $\mathfrak{n}$ (see $[16, \S 3.2]$ for the case $F=\mathbb{Q}$ ).

Proof. - Since $\bar{\rho}$ is totally odd, if it is irreducible, then it is absolutely irreducible. Assume that $\bar{\rho}$ is reducible: $\bar{\rho}^{\text {s.s. }}=\varphi_{\text {gal }} \oplus \varphi_{\text {gal }}^{\prime}$. The characters $\varphi_{\text {gal }}, \varphi_{\text {gal }}^{\prime}: \mathcal{G}_{F} \rightarrow \kappa^{\times}$are unramified outside $\mathfrak{n} p$ and $\varphi_{\text {gal }} \varphi_{\text {gal }}^{\prime}=\operatorname{det}(\bar{\rho})=\bar{\psi}_{\text {gal }} \omega^{-1}$ (recall that $\psi$ is a Hecke character of infinity type $-n_{0} t$ ). Denote by $\hat{\mathfrak{o}}_{\mathfrak{n}, 1}^{\times}$the subgroup of $\hat{\mathfrak{o}}^{\times}$of elements $\equiv 1(\bmod \mathfrak{n})$. Then $\hat{\mathfrak{o}}_{\mathfrak{n}, 1}^{\times}$is a product of its $p$-part $\prod_{\mathfrak{p} \mid p} \mathfrak{o}_{\mathfrak{p}}^{\times}$and its part outside $p$, denoted by $\hat{\mathfrak{o}}_{\mathfrak{n}, 1}^{\times(p)}$.

By the global class field theory, the Galois group of the maximal $\mathfrak{n}$-ramified (respectively $\mathfrak{n} p^{\infty}$ ramified) Abelian extension of $F$ is isomorphic to $\mathrm{Cl}_{F, \mathfrak{n}}^{+}=\mathbb{A}_{F}^{\times} / F^{\times} \hat{\mathfrak{o}}_{\mathfrak{n}, 1}^{\times} D(\mathbb{R})_{+}$(respectively $\left.\mathrm{Cl}_{F, \mathfrak{n} p^{\infty}}^{+}:=\lim _{\leftarrow} \mathrm{Cl}_{F, \mathfrak{n} p^{r}}^{+}=\mathbb{A}_{F}^{\times} / \overline{F^{\times} \hat{\mathfrak{o}}_{\mathfrak{n}, 1}^{\times(p)} D(\mathbb{R})_{+}}\right)$. We choose the convention in which an uniformizer corresponds to a geometric Frobenius. We have the following exact sequence

$$
1 \rightarrow\left(\prod_{\mathfrak{p} \mid p} \mathfrak{o}_{\mathfrak{p}}^{\times}\right) / \overline{\left\{\epsilon \in \mathfrak{o}_{+}^{\times} \mid \epsilon-1 \in \mathfrak{n}\right\}} \rightarrow \mathrm{Cl}_{F, \mathfrak{n} p \infty}^{+} \rightarrow \mathrm{Cl}_{F, \mathfrak{n}}^{+} \rightarrow 1 .
$$

By Corollary 2.13, for each

$$
\mathfrak{p} \mid p, \varphi_{\text {gal }} \oplus \varphi_{\text {gal }}^{\prime}
$$

is crystalline at $\mathfrak{p}$ of weights $\left(m_{\tau}, k_{0}-m_{\tau}-1\right)_{\tau \in J_{F, \mathfrak{p}}}$.

By (13) for every $\epsilon \in \mathfrak{o}_{+}^{\times}, \epsilon-1 \in \mathfrak{n}$ we have the following equality in $\kappa$ :

$$
1=\varphi_{\text {gal }}(\epsilon)=\prod_{\mathfrak{p} \mid p} \varphi_{\text {gal }, \mathfrak{p}}(\epsilon)=\prod_{\mathfrak{p} \mid p} \prod_{\tau \in J_{F, \mathfrak{p}}} \tau(\epsilon)^{m_{\tau} \text { or }\left(k_{0}-m_{\tau}-1\right)}=\epsilon^{p(J)},
$$

for some subset $J \subset J_{F}$. By the assumption $p>k_{0}$, if $k$ is non-parallel, then $\epsilon^{p(J)} \neq 1$ for all $J \subset J_{F}$. Thus we obtain (ii) and (i) when $k$ is non-parallel.

$4^{\mathrm{e}}$ SÉRIE - TOME $38-2005-\mathrm{N}^{\circ} 4$ 
Assume now that $k=k_{0} t$ is parallel and that for all $\emptyset \subsetneq J \subsetneq J_{F}$, there exists $\epsilon \in \mathfrak{o}_{+}^{\times}, \epsilon-1 \in \mathfrak{n}$, such that $p$ does not divide the non-zero integer $\mathrm{N}_{F / \mathbb{Q}}\left(\epsilon^{p(J)}-1\right)$. The same arguments as above show that the restriction to $\prod_{\mathfrak{p} \mid p} \mathfrak{o}_{\mathfrak{p}}^{\times}$of the character $\varphi_{\text {gal }}$ (respectively $\varphi_{\text {gal }}^{\prime}$ ) $\mathrm{Cl}_{F, \mathfrak{n} p \infty}^{+} \rightarrow \kappa^{\times}$is trivial (respectively given by the $\left(1-k_{0}\right)$ th power of the norm). By the following lemma (applied to $P=\mathrm{Cl}_{F, \mathfrak{n} p \infty}^{+}$and $Q=\left(\prod_{\mathfrak{p} \mid p} \mathfrak{o}_{\mathfrak{p}}^{\times}\right) / \overline{\left\{\epsilon \in \mathfrak{o}_{+}^{\times} \mid \epsilon-1 \in \mathfrak{n}\right\}}$ ) there exists a unique character $\tilde{\varphi}_{\text {gal }}$ (respectively $\left.\tilde{\varphi}_{\text {gal }}^{\prime}\right): \mathrm{Cl}_{F, \mathfrak{n} p \infty}^{+} \rightarrow \mathcal{O}^{\times}$lifting $\varphi_{\text {gal }}$ (respectively $\varphi_{\text {gal }}^{\prime}$ ) and whose restriction to $\prod_{\mathfrak{p} \mid p} \mathfrak{o}_{\mathfrak{p}}^{\times}$is trivial (respectively given by the $\left(1-k_{0}\right)$ th power of the norm).

Lemma 3.3. - Let $P$ be an Abelian group and $Q$ be a subgroup, such that the factor group $P / Q$ is finite. Let $\varphi_{P}: P \rightarrow \kappa^{\times}$and $\tilde{\varphi}_{Q}: Q \rightarrow \mathcal{O}^{\times}$be two characters such that we have $\left.\varphi_{P}\right|_{Q}=\tilde{\varphi}_{Q} \bmod p$. Then, there exists a unique character $\tilde{\varphi}_{P}: P \rightarrow \mathcal{O}^{\times}$, whose restriction to $Q$ is $\tilde{\varphi}_{Q}$ and such that $\tilde{\varphi}_{P} \bmod p=\varphi_{P}$.

For $x \in \mathbb{A}_{F}^{\times}$, we put $\varphi(x):=\tilde{\varphi}_{\text {gal }}(x)$ and $\varphi^{\prime}(x):=\tilde{\varphi}_{\text {gal }}^{\prime}(x) x_{p}^{-k} x_{\infty}^{k}$. Then $\varphi\left(\right.$ respectively $\left.\varphi^{\prime}\right)$ is a Hecke character of $F$, of conductor dividing $\mathfrak{n}$ and infinity type 0 (respectively $\left(1-k_{0}\right) t$ ). It is crucial to observe that there are only finitely many such $\varphi$ and $\varphi^{\prime}$.

Assume now that for infinitely many primes $p, \bar{\rho}$ is reducible. Then there exist Hecke characters $\varphi$ and $\varphi^{\prime}$ as above, such that for infinitely many primes $p$ we have $\bar{\rho}^{\text {s.s. }} \equiv \varphi_{\text {gal }} \oplus \varphi_{\text {gal }}^{\prime}$ $(\bmod \mathcal{P})$. Hence for each prime $v$ of $F$ not dividing $\mathfrak{n}$ we have $c(f, v) \equiv \varphi\left(\varpi_{v}\right)+\varphi^{\prime}\left(\varpi_{v}\right)$ $(\bmod \mathcal{P})$ for infinitely many $\mathcal{P}$ 's and hence $c(f, v)=\varphi\left(\varpi_{v}\right)+\varphi^{\prime}\left(\varpi_{v}\right)$. By the Cebotarev Density Theorem we obtain $\rho^{\text {s.s. }}=\varphi \oplus \varphi^{\prime}$. This contradicts the irreducibility of $\rho$.

\subsection{The exceptional case}

The aim of this paragraph is to find a bound for the primes $p$ such that $\operatorname{pr}\left(\bar{\rho}\left(\mathcal{G}_{F}\right)\right)$ is isomorphic to one of the groups $A_{4}, S_{4}$ or $A_{5}$. We will only use the fact that the elements of these groups are of order at most 5 .

Assume that $\operatorname{pr}\left(\bar{\rho}\left(\mathcal{G}_{F}\right)\right) \cong A_{4}, S_{4}$ or $A_{5}$. By Corollary 2.13 there exist $\epsilon_{\tau} \in\{ \pm 1\}, \tau \in J_{F}$, such that for all $\mathfrak{p} \mid p$ and for any generator $x$ of $\mathbb{F}_{p^{h}}^{\times}$, where $h=\left|J_{F, \mathfrak{p}}\right|$, the element

$$
\prod_{\tau \in \operatorname{Gal}\left(\mathbb{F}_{p^{h}} / \mathbb{F}_{p}\right)} \tau(x)^{\epsilon_{\tau}\left(k_{\tau}-1\right)} \in \mathbb{F}_{p^{h}}^{\times}
$$

belongs to $\operatorname{pr}\left(\bar{\rho}\left(I_{\mathfrak{p}}\right)\right)$ and is therefore of order at most 5 (if (ORD) holds we may assume that $\epsilon_{\tau}=1$ for all $\tau$ ). Denote by $\tau_{1}, \ldots, \tau_{h}$ the elements of $J_{F, \mathfrak{p}}$. Then

$$
\epsilon_{\tau_{1}}\left(k_{\tau_{1}}-1\right)+\epsilon_{\tau_{2}} p\left(k_{\tau_{2}}-1\right)+\cdots+\epsilon_{\tau_{h}} p^{h-1}\left(k_{\tau_{h}}-1\right) \in \mathbb{Z} /\left(p^{h}-1\right)
$$

is of order $\leqslant 5$, hence $5\left(\left(k_{\tau_{1}}-1\right)+p\left(k_{\tau_{2}}-1\right)+\cdots+p^{h-1}\left(k_{\tau_{h}}-1\right)\right) \geqslant p^{h}-1$.

If we replace the generator $x$ by $x^{p}, x^{p^{2}}, \ldots, x^{p^{h-1}}$ and then sum these inequalities we find $5 \sum_{\tau \in J_{F, \mathfrak{p}}}\left(k_{\tau}-1\right) \geqslant\left|J_{F, \mathfrak{p}}\right|(p-1)$. Hence $5 \sum_{\tau \in J_{F}}\left(k_{\tau}-1\right) \geqslant d(p-1)$.

We conclude that $\operatorname{pr}\left(\bar{\rho}\left(\mathcal{G}_{F}\right)\right)$ cannot be isomorphic to $A_{4}, S_{4}$ or $A_{5}$ if

$$
d(p-1)>5 \sum_{\tau \in J_{F}}\left(k_{\tau}-1\right) .
$$

Note that this assumption follows from (II) if $d \geqslant 5$. 


\subsection{The dihedral case}

In this paragraph we study the case when $\operatorname{pr}\left(\bar{\rho}\left(\mathcal{G}_{F}\right)\right)$ is isomorphic to the dihedral group $D_{2 r}$, where $r \geqslant 3$ is an integer prime to $p$. Let $C_{r}$ be the cyclic subgroup of order $r$ of $D_{2 r}$. Since $\operatorname{pr}^{-1}\left(C_{r}\right)$ is a commutative group containing only semi-simple elements ( $p$ does not divide $r$ ), it is diagonalizable. Since $\mathrm{pr}^{-1}\left(D_{2 r} \backslash C_{r}\right)$ is contained in the normalizer of $\mathrm{pr}^{-1}\left(C_{r}\right)$, it is contained in the set of anti-diagonal matrices.

Let $\varepsilon: D_{2 r} \rightarrow\{ \pm 1\}$ be the sign map and let $K$ be the fixed field of $\operatorname{ker}(\varepsilon \circ \operatorname{pr} \circ \bar{\rho})$. The extension $K / F$ is quadratic and unramified outside $\mathfrak{n} p$.

Let $c$ be the non-trivial element of the Galois group $\operatorname{Gal}(K / F)$. Since $\bar{\rho}$ is absolutely irreducible, but $\bar{\rho}_{\mid \mathcal{G}_{K}}$ is not, there exists a character $\varphi_{\text {gal }}: \mathcal{G}_{K} \rightarrow \kappa^{\times}$distinct from its Galois conjugate $\varphi_{\text {gal }}^{c}$ and such that $\bar{\rho}_{\mid \mathcal{G}_{K}}=\varphi_{\text {gal }} \oplus \varphi_{\text {gal }}^{c}$.

LEMMA 3.4. - Let $\mathfrak{p}$ be a prime of $F$ dividing $p$. Assume that there exists $\tau \in J_{F, \mathfrak{p}}$ such that $p \neq 2 k_{\tau}-1$. Then the field $K$ is unramified at $\mathfrak{p}$, and $\varphi_{\text {gal }}$ is crystalline at primes $\mathfrak{P}$ of $K$ above $\mathfrak{p}$ of weights $\left(m_{\tau}, k_{0}-m_{\tau}-1\right)_{\tau \in J_{F, \mathfrak{p}}}$.

Proof. - If $K / F$ ramifies at $\mathfrak{p}$ then $\bar{\rho}\left(I_{\mathfrak{p}}\right)$ would contain at least one anti-diagonal matrix and the basis vectors would not be eigen for $\bar{\rho}\left(I_{\mathfrak{p}}\right)$. But the group $\bar{\rho}\left(I_{\mathfrak{p}}\right)$ has a common eigenvector. Hence, the elements of $\operatorname{pr}\left(\bar{\rho}\left(I_{\mathfrak{p}}\right)\right)$ would be of order $\leqslant 2$. Using the computations of Section 3.2 and $p>k_{0}$, we find that for all $\tau \in J_{F, \mathfrak{p}}$ we have $2\left(k_{\tau}-1\right)=p-1$.

By Corollary 2.13, $\varphi_{\text {gal }} \oplus \varphi_{\text {gal }}^{c}$ is crystalline at $\mathfrak{P}$ of weights $\left(m_{\tau}, k_{0}-m_{\tau}-1\right)_{\tau \in J_{F, \mathfrak{p}}}$ and $\left(\varphi_{\text {gal }} \varphi_{\text {gal }}^{c}\right)_{\mid I_{\mathfrak{P}}}=\omega_{\mid I_{\mathfrak{P}}}^{1-k_{0}}$.

Let $\mathfrak{O}$ be the ring of integers of $K$, and $\widehat{\mathfrak{O}}$ its profinite completion. Denote by $\widehat{\mathfrak{O}}_{\mathfrak{n}, 1}^{\times}$the subgroup of $\widehat{\mathfrak{D}}^{\times}$of elements $\equiv 1(\bmod \mathfrak{n})$. Then $\widehat{\mathfrak{D}}_{\mathfrak{n}, 1}^{\times}$is a product of its $p$-part $\prod_{\mathfrak{P} \mid p} \mathfrak{D}_{\mathfrak{P}}^{\times}$and its part outside $p$, denoted by $\widehat{\mathfrak{O}}_{\mathfrak{n}, 1}^{\times(p)}$.

By the global class field theory, the Galois group of the maximal $\mathfrak{n}$-ramified (respectively $\mathfrak{n} p^{\infty}$. ramified) Abelian extension of $K$ is isomorphic to $\mathrm{Cl}_{K, \mathfrak{n}}:=\mathbb{A}_{K}^{\times} / K^{\times} \widehat{\mathfrak{O}}_{\mathfrak{n}, 1}^{\times} K_{\infty}^{\times}$(respectively to $\mathrm{Cl}_{K, \mathfrak{n} p \infty}:=\mathbb{A}_{K}^{\times} / \overline{\left.K^{\times} \widehat{\mathfrak{O}}_{\mathfrak{n}, 1}^{\times(p)} K_{\infty}^{\times}\right)}$. We have the following exact sequence:

$$
1 \rightarrow\left(\prod_{\mathfrak{P} \mid p} \mathfrak{O}_{\mathfrak{P}}^{\times}\right) / \overline{\left\{\epsilon \in \mathfrak{O}^{\times} \mid \epsilon-1 \in \mathfrak{n}\right\}} \rightarrow \mathrm{Cl}_{K, \mathfrak{n} p \infty} \rightarrow \mathrm{Cl}_{K, \mathfrak{n}} \rightarrow 1
$$

PROPOSITION 3.5. -

(i) Assume that for all $\tau \in J_{F}, p \neq 2 k_{\tau}-1$ and that $\operatorname{pr}\left(\bar{\rho}\left(\mathcal{G}_{F}\right)\right)$ is dihedral. Let $K / F$ be the quadratic extension defined above. Then one of the following holds:

- $K$ is $C M$ and there exists a Hecke character $\varphi$ of $K$ of conductor of norm dividing $\mathfrak{n} \Delta_{K / F}^{-1}$ and infinity type $\left(m_{\tau}, k_{0}-1-m_{\tau}\right)_{\tau \in J_{F}}$ such that $\rho \equiv \operatorname{Ind}_{K}^{F} \varphi(\bmod \mathcal{P})$,

- $K$ is not $C M$ and we can choose places $\tilde{\tau}$ of $K$ above each $\tau \in J_{F}$ such that for all $\epsilon \in \mathfrak{O}^{\times}, \epsilon-1 \in \mathfrak{n}$ the prime $p$ divides $\mathrm{N}_{K / \mathbb{Q}}\left(\prod_{\tau \in J_{F}} \tilde{\tau}(\epsilon)^{m_{\tau}} \tilde{\tau}(c(\epsilon))^{k_{0}-m_{\tau}-1}-1\right)$.

(ii) Assume that $f$ is not a theta series. Then for all but finitely many primes $p$ the group $\operatorname{pr}\left(\bar{\rho}\left(\mathcal{G}_{F}\right)\right)$ is not dihedral.

Remark 3.6. -

(i) The primes $p$ for which the congruence $\rho \equiv \operatorname{Ind}_{K}^{F} \varphi(\bmod \mathcal{P})$ may occur should be controlled by the value at 1 of the $L$-function associated to the CM character $\varphi / \varphi^{c}$ (in the elliptic case it is proved by Hida [22] and Ribet [36]; see also Theorems A and B). 
(ii) We would like to thank E. Ghate for having pointed us out the possible existence of dihedral primes for non-CM fields $K$. It would be interesting to explore the converse statement, that is to say to try to construct for a given prime $p$ dividing the above norms, a newform $f$ such that $\operatorname{pr}\left(\bar{\rho}\left(\mathcal{G}_{F}\right)\right)$ is dihedral.

Proof. - (i) By (14) and the above lemma, we have $\varphi_{\text {gal }}: \mathrm{Cl}_{K, \mathfrak{n} p \infty} \rightarrow \kappa^{\times}$whose restriction to $\prod_{\mathfrak{P} \mid p} \mathfrak{O}_{\mathfrak{P}}^{\times}$is given by the reduction modulo $p$ of an algebraic character $x \mapsto x^{\tilde{k}}$, where $\tilde{k}=\sum_{\tau \in J_{F}} m_{\tau} \tilde{\tau}+\left(k_{0}-m_{\tau}-1\right) \tilde{\tau} \circ c$, for some choice of places $\tilde{\tau}$ of $K$ above $\tau \in J_{F}$.

We observe that the character $x \mapsto x^{\tilde{k}}$ is trivial on $\mathfrak{o}_{+}^{\times}$, whereas it is only trivial modulo $p$ on $\left\{\epsilon \in \mathfrak{O}^{\times} \mid \epsilon-1 \in \mathfrak{n}\right\}$. The case when $K$ is not CM follows immediately.

Assume now that $K$ is CM. In this case $\left\{\epsilon \in \mathfrak{o}_{+}^{\times} \mid \epsilon-1 \in \mathfrak{n}\right\}$ is a finite index subgroup of $\left\{\epsilon \in \mathfrak{O}^{\times} \mid \epsilon-1 \in \mathfrak{n}\right\}$. Since $\operatorname{ker}\left(\mathcal{O}^{\times} \rightarrow \kappa^{\times}\right)$does not contain elements of finite order, the above character is trivial on $\left\{\epsilon \in \mathfrak{O}^{\times} \mid \epsilon-1 \in \mathfrak{n}\right\}$.

By Lemma 3.3 (applied to $P=\mathrm{Cl}_{K, \mathfrak{n} p^{\infty}}$ and $Q=\left(\prod_{\mathfrak{P} \mid p} \mathfrak{O}_{\mathfrak{P}}^{\times}\right) / \overline{\left\{\epsilon \in \mathfrak{O}^{\times} \mid \epsilon-1 \in \mathfrak{n}\right\}}$ ) there exists a lift $\tilde{\varphi}_{\text {gal }}: \mathrm{Cl}_{K, \mathfrak{n} p \infty} \rightarrow \mathcal{O}^{\times}$whose restriction to $\prod_{\mathfrak{P} \mid p} \mathfrak{O}_{\mathfrak{P}}^{\times}$is given by $x \mapsto x^{\tilde{k}}$.

We put $\varphi(x):=\tilde{\varphi}_{\text {gal }}(x) x_{p}^{-\tilde{k}} x_{\infty}^{\tilde{k}}$. Then $\varphi$ is a Hecke character of $K$ as desired.

(ii) There are finitely many fields $K$ as above. For those $K$ that are not CM it is enough to choose $\epsilon \in \mathfrak{O}^{\times}, \epsilon-1 \in \mathfrak{n}$, of infinite order in $\mathfrak{O}^{\times} / \mathfrak{o}^{\times}$.

For each of the CM fields $K$ that are only finitely many characters $\varphi$ as above. Therefore, if $\operatorname{pr}\left(\bar{\rho}\left(\mathcal{G}_{F}\right)\right)$ is dihedral for infinitely many primes $p$, then there would exist $K$ and $\varphi$ as above, such that the congruence $\rho \equiv \operatorname{Ind}_{K}^{F} \varphi(\bmod \mathcal{P})$ happens for infinitely many $\mathcal{P}$ 's. Hence $f$ would be equal to the theta series associated to $\varphi$.

\subsection{The image of $\bar{\rho}$ is "large"}

THEOREM 3.7 (Dickson). -

(i) An irreducible subgroup of $\mathrm{PSL}_{2}(\kappa)$ of order divisible by $p$ is conjugated inside $\mathrm{PGL}_{2}(\kappa)$ to $\mathrm{PSL}_{2}\left(\mathbb{F}_{q}\right)$ or to $\mathrm{PGL}_{2}\left(\mathbb{F}_{q}\right)$, for some power $q$ of $p$.

(ii) An irreducible subgroup of $\mathrm{PSL}_{2}(\kappa)$ of order prime to $p$ is either dihedral, or isomorphic to one of the groups $A_{4}, S_{4}$ or $A_{5}$.

As an application of this theorem, Propositions 3.1, 3.5 and Section 3.2 we obtain the following

Proposition 3.8. - Assume that $f \in S_{k}(\mathfrak{n}, \psi)$ is a newform, which is not a theta series. Then for all but finitely many primes $p$, the image of $\bar{\rho}$ is large in the following sense:

$\left(\mathbf{L} \mathbf{I}_{\bar{\rho}}\right)$ there exists a power $q$ of $p$ such that $\mathrm{SL}_{2}\left(\mathbb{F}_{q}\right) \subset \operatorname{im}(\bar{\rho}) \subset \kappa^{\times} \mathrm{GL}_{2}\left(\mathbb{F}_{q}\right)$.

Let $\widehat{F}$ be the compositum of $\widetilde{F}$ and of the subfield of $\overline{\mathbb{Q}}$ fixed by the Galois group $\operatorname{ker}\left(\bar{\psi} \omega^{n_{0}}\right)$. The extension $\widehat{F} / F$ is Galois and unramified at $p$, since $\widetilde{F}$ is unramified at $p$ and $\psi$ is of conductor prime to $p$. Therefore $\mathcal{G}_{\widehat{F}}$ is a normal subgroup of $\mathcal{G}_{F}$ containing the inertia subgroups $I_{\mathfrak{p}}$, for all $\mathfrak{p}$ dividing $p$.

We put $\mathcal{D}=\operatorname{det}\left(\bar{\rho}\left(\mathcal{G}_{\widehat{F}}\right)\right)=\left(\mathbb{F}_{p}^{\times}\right)^{1-k_{0}}$.

Proposition 3.9. - Assume $\left(\mathbf{L I}_{\bar{\rho}}\right)$. Then there exists a power $q$ of $p$ such that, either

$$
\begin{aligned}
& \bar{\rho}\left(\mathcal{G}_{\widehat{F}}\right)=\mathrm{GL}_{2}\left(\mathbb{F}_{q}\right)^{\mathcal{D}}:=\left\{x \in \mathrm{GL}_{2}\left(\mathbb{F}_{q}\right) \mid \operatorname{det}(x) \in \mathcal{D}\right\}, \quad \text { or } \\
& U U \bar{\rho}\left(\mathcal{G}_{\widehat{F}}\right)=\left(\mathbb{F}_{q^{2}}^{\times} \mathrm{GL}_{2}\left(\mathbb{F}_{q}\right)\right)^{\mathcal{D}}:=\left\{x \in \mathbb{F}_{q^{2}}^{\times} \mathrm{GL}_{2}\left(\mathbb{F}_{q}\right) \mid \operatorname{det}(x) \in \mathcal{D}\right\} .
\end{aligned}
$$

Proof. - We first show that $\operatorname{pr}\left(\bar{\rho}\left(\mathcal{G}_{\widehat{F}}\right)\right)$ is still irreducible of order divisible by $p$. By $\left(\mathbf{L I}_{\bar{\rho}}\right)$ the group $\operatorname{pr}\left(\bar{\rho}\left(\mathcal{G}_{F}\right)\right)$ is isomorphic to $\mathrm{PSL}_{2}\left(\mathbb{F}_{q}\right)$ or $\mathrm{PGL}_{2}\left(\mathbb{F}_{q}\right)$. The group $\operatorname{pr}\left(\bar{\rho}\left(\mathcal{G}_{\widehat{F}}\right)\right)$ is a non-trivial 
normal subgroup of $\operatorname{pr}(\operatorname{im}(\bar{\rho}))$ (because it contains $\operatorname{pr}\left(\bar{\rho}\left(I_{p}\right)\right)$ and $p>k_{0}$; see Corollary 2.13). Since $\mathrm{PSL}_{2}\left(\mathbb{F}_{q}\right)$ is a simple group of index 2 in the group $\mathrm{PGL}_{2}\left(\mathbb{F}_{q}\right)$, we deduce

$$
\operatorname{PSL}_{2}\left(\mathbb{F}_{q}\right) \subset \operatorname{pr}\left(\bar{\rho}\left(\mathcal{G}_{\widehat{F}}\right)\right) \subset \operatorname{pr}\left(\bar{\rho}\left(\mathcal{G}_{F}\right)\right) \subset \operatorname{PGL}_{2}\left(\mathbb{F}_{q}\right) .
$$

LEMmA 3.10. - Let $H$ be a group of center $Z$ and let $\mathrm{pr}: H \rightarrow H / Z$ the canonical projection. Let $P$ and $Q$ be two subgroups of $H$ such that $\operatorname{pr}(P) \supset \operatorname{pr}(Q)$. Assume moreover that $Q$ does not have non-trivial Abelian quotients. Then $P \supset Q$.

It follows from this lemma that $\bar{\rho}\left(\mathcal{G}_{\widehat{F}}\right) \supset \mathrm{SL}_{2}\left(\mathbb{F}_{q}\right)$, hence

$$
\left(\kappa^{\times} \mathrm{GL}_{2}\left(\mathbb{F}_{q}\right)\right)^{\mathcal{D}} \supset \bar{\rho}\left(\mathcal{G}_{\widehat{F}}\right) \supset \mathrm{GL}_{2}\left(\mathbb{F}_{q}\right)^{\mathcal{D}} \text {. }
$$

Since $\left[\left(\kappa^{\times} \mathrm{GL}_{2}\left(\mathbb{F}_{q}\right)\right)^{\mathcal{D}}: \mathrm{GL}_{2}\left(\mathbb{F}_{q}\right)^{\mathcal{D}}\right] \leqslant 2$ we are done.

Let $\theta \in \mathbb{F}_{q^{2}} \backslash \mathbb{F}_{q}$ be such that $\theta^{2} \in \mathbb{F}_{q}$. Then $\left(\mathbb{F}_{q^{2}}^{\times} \mathrm{GL}_{2}\left(\mathbb{F}_{q}\right)\right)^{\mathcal{D}}=\mathrm{GL}_{2}\left(\mathbb{F}_{q}\right)^{\mathcal{D}} \amalg\left(\theta \mathrm{GL}_{2}\left(\mathbb{F}_{q}\right)\right)^{\mathcal{D}}$ and hence $\operatorname{tr}\left(\left(\mathbb{F}_{q^{2}}^{\times} \mathrm{GL}_{2}\left(\mathbb{F}_{q}\right)\right)^{\mathcal{D}}\right)=\mathbb{F}_{q} \cup \theta \mathbb{F}_{q}$. Therefore, the $\mathbb{F}_{p}$-algebra generated by the traces of the elements of $\left(\mathbb{F}_{q^{2}}^{\times} \mathrm{GL}_{2}\left(\mathbb{F}_{q}\right)\right)^{\mathcal{D}}$ is $\mathbb{F}_{q^{2}}$, while $\operatorname{pr}\left(\left(\mathbb{F}_{q^{2}}^{\times} \mathrm{GL}_{2}\left(\mathbb{F}_{q}\right)\right)^{\mathcal{D}}\right) \subset \mathrm{PGL}_{2}\left(\mathbb{F}_{q}\right)$. This reflects the existence of a congruence with a form having inner twists.

\subsection{The image of $\operatorname{Ind}_{F}^{\mathbb{Q}} \bar{\rho}$ is "large"}

We assume in this paragraph that $\left(\mathbf{L I}_{\bar{\rho}}\right)$ holds.

By Proposition 3.9 there exists a power $q$ of $p$ such that $\operatorname{pr}\left(\bar{\rho}\left(\mathcal{G}_{\widehat{F}}\right)\right)=\operatorname{PSL}_{2}\left(\mathbb{F}_{q}\right)$ or $\mathrm{PGL}_{2}\left(\mathbb{F}_{q}\right)$. Consider the representation $\operatorname{pr}\left(\operatorname{Ind}_{F}^{\mathbb{Q}} \bar{\rho}\right): \mathcal{G}_{\widehat{F}} \rightarrow \mathrm{PGL}_{2}\left(\mathbb{F}_{q}\right)^{J_{F}}$. An automorphism of the simple group $\mathrm{PSL}_{2}\left(\mathbb{F}_{q}\right)$ is a composition of a conjugation by an element of $\mathrm{PGL}_{2}\left(\mathbb{F}_{q}\right)$ with an automorphism of $\mathbb{F}_{q}$. By a lemma of Serre (see [35]), there exist a partition $J_{F}=\coprod_{i \in I} J_{F}^{i}$ and for all $i \in I, \tau \in J_{F}^{i}$, an element $\sigma_{i, \tau} \in \operatorname{Gal}\left(\mathbb{F}_{q} / \mathbb{F}_{p}\right)$ such that

$$
\operatorname{pr}\left(\phi\left(\mathrm{SL}_{2}\left(\mathbb{F}_{q}\right)^{I}\right)\right) \subset \operatorname{pr}\left(\operatorname{Ind}_{F}^{\mathbb{Q}} \bar{\rho}\left(\mathcal{G}_{\widehat{F}}\right)\right) \subset \operatorname{pr}\left(\phi\left(\mathrm{GL}_{2}\left(\mathbb{F}_{q}\right)^{I}\right)\right)
$$

where $\phi=\left(\phi^{i}\right)_{i \in I}: \mathrm{GL}_{2}\left(\mathbb{F}_{q}\right)^{I} \hookrightarrow \mathrm{GL}_{2}\left(\mathbb{F}_{q}\right)^{J_{F}}$ is given by $\phi^{i}\left(M_{i}\right)=\left(M_{i}^{\sigma_{i, \tau}}\right)_{\tau \in J_{F}^{i}}$.

Keeping these notations, we introduce the following assumption on the image of $\operatorname{Ind}_{F}^{\mathbb{Q}} \bar{\rho}$ :

$\left(\mathbf{L} \mathbf{I}_{\text {Ind } \bar{\rho}}\right)$ the condition $\left(\mathbf{L I}_{\bar{\rho}}\right)$ holds and $\forall i \in I, \forall \tau, \tau^{\prime} \in J_{F}^{i}\left(\tau \neq \tau^{\prime} \Longrightarrow \sigma_{i, \tau} \neq \sigma_{i, \tau^{\prime}}\right)$.

We now introduce a genericity assumption on the weight $k$.

DEFINITION 3.11. - We say that the weight $k \in \mathbb{Z}\left[J_{F}\right]$ is non-induced, if there do not exist a strict subfield $F^{\prime}$ of $F$ and a weight $k^{\prime} \in \mathbb{Z}\left[J_{F^{\prime}}\right]$ such that for each $\tau \in J_{F}, k_{\tau}=k_{\left.\tau\right|_{F^{\prime}}}^{\prime}$.

Remark 3.12. - Define $\tilde{k}=\sum_{\tilde{\tau} \in J_{\widetilde{F}}} k_{\tilde{\tau}} \tilde{\tau} \in \mathbb{Z}\left[J_{\widetilde{F}}\right]$ by putting $k_{\tilde{\tau}}=k_{\left.\tilde{\tau}\right|_{F}}$, for all $\tilde{\tau} \in J_{\widetilde{F}}$. The group $\mathcal{G}_{\mathbb{Q}}$ acts on $\mathbb{Z}\left[J_{\widetilde{F}}\right]$ by $\tilde{k}=\sum_{\tilde{\tau} \in J_{\widetilde{F}}} k_{\tilde{\tau}} \tilde{\tau} \mapsto \tilde{k}^{\tilde{\tau}^{\prime}}=\sum_{\tilde{\tau} \in J_{\widetilde{F}}} k_{\tilde{\tau} \tilde{\tau}} \tilde{\tau}^{\prime}$. It is easy to see that $k \in \mathbb{Z}\left[J_{F}\right]$ is non-induced if, and only if, $\left\{\tilde{\tau}^{\prime} \in \mathcal{G}_{\mathbb{Q}} \mid \tilde{k}=\tilde{k}^{\tilde{\tau}^{\prime}}\right\}$ equals $\mathcal{G}_{F}$.

PROPOSITION 3.13. - Assume that $\left(\mathbf{L I}_{\bar{\rho}}\right)$ holds and $k$ is non-induced. Assume moreover that for all $\tau \neq \tau^{\prime} \in J_{F}, p \neq k_{\tau}+k_{\tau^{\prime}}-1$. Then $\left(\mathbf{L I}_{\text {Ind } \bar{\rho}}\right)$ holds.

Proof. - Let $\tilde{\tau}_{1}, \tilde{\tau}_{2} \in \mathcal{G}_{\mathbb{Q}}$ be such that for all $y \in \mathcal{G}_{\widehat{F}}$ we have $\operatorname{pr}\left(\bar{\rho}\left(\tilde{\tau}_{1}^{-1} y \tilde{\tau}_{1}\right)\right)=\operatorname{pr}\left(\bar{\rho}\left(\tilde{\tau}_{2}^{-1} y \tilde{\tau}_{2}\right)\right)$. We have to prove that $\tilde{\tau}_{1}^{-1} \tilde{\tau}_{2} \in \mathcal{G}_{F}$. For $i=1,2$, let $\bar{\rho}_{i}(y)=\bar{\rho}\left(\tilde{\tau}_{i}^{-1} y \tilde{\tau}_{i}\right)$. 
Let $\mathfrak{P}$ be a prime ideal of $\widehat{F}$ above a prime ideal $\mathfrak{p}$ of $F$ dividing $p$. By Corollary 2.13 we have $\left.\bar{\rho}_{i}\right|_{I_{\mathfrak{P}}} ^{\text {s.s. }}=\varepsilon_{i} \oplus \delta_{i}$, where $\varepsilon_{i}$ and $\delta_{i}$ are two tame characters whose product equals $\omega^{1-k_{0}}$ and whose sum has Fontaine-Laffaille weights $\left(m_{\tau}, k_{0}-m_{\tau}-1\right)_{\tau \in J_{F, p_{i}}}$.

Since $I_{\mathfrak{P}} \subset \mathcal{G}_{\widehat{F}}$ and $\operatorname{pr} \circ \bar{\rho}_{1}=\operatorname{pr} \circ \bar{\rho}_{2}$ on $\mathcal{G}_{\widehat{F}}$, we have $\varepsilon_{1} / \delta_{1}=\varepsilon_{2} / \delta_{2}$. By varying $\mathfrak{P}$ we deduce that for all $\tilde{\tau} \in J_{\widetilde{F}}, \tilde{k}_{\tilde{\tau}}=\tilde{k}_{\tilde{\tau} \tilde{\tau}_{1}^{-1} \tilde{\tau}_{2}}$ (here we use that $p>k_{0}$ and $p \neq k_{\tau}+k_{\tau^{\prime}}-1$ ). Since $k$ is noninduced, it follows from Remark 3.12 that $\tilde{\tau}_{1}^{-1} \tilde{\tau}_{2} \in \mathcal{G}_{F}$.

The following corollary generalizes a result of Ribet [35] on the image of a Galois representation associated to a family of classical modular forms, to the case of the family of internal conjugates of a Hilbert modular form.

COROLlary 3.14. - Assume that $\left(\mathbf{L I}_{\bar{\rho}}\right)$ holds and $k$ is non-induced. Assume moreover that $p>2 k_{0}$ is totally split in $F$. Then,

$$
\left(\operatorname{GL}_{2}\left(\mathbb{F}_{q}\right)^{J_{F}}\right)^{\mathcal{D}} \subset \operatorname{Ind}_{F}^{\mathbb{Q}} \bar{\rho}\left(\mathcal{G}_{\widehat{F}}\right) \subset\left(\bar{\rho}\left(\mathcal{G}_{\widehat{F}}\right)^{J_{F}}\right)^{\mathcal{D}}, \quad \text { where } \mathcal{D}=\left(\mathbb{F}_{p}^{\times}\right)^{1-k_{0}} .
$$

Put

$$
H\left(\mathbb{F}_{q}\right)=\left(\prod_{i \in I} \mathrm{GL}_{2}\left(\mathbb{F}_{q}\right)\right)^{\mathcal{D}}:=\left\{\left(M_{i}\right)_{i \in I} \in \prod_{i \in I} \mathrm{GL}_{2}\left(\mathbb{F}_{q}\right) \mid \exists \delta \in \mathcal{D}, \forall i, \operatorname{det}\left(M_{i}\right)=\delta\right\} .
$$

LEMma 3.15. - Assume that $\left(\mathbf{L I}_{\bar{\rho}}\right)$ holds and $p>2 k_{0}$. Then,

(i) for all $\mathfrak{p}$ dividing $p, \bar{\rho}\left(I_{\mathfrak{p}}\right)$ is contained (possibly after conjugation by an element of $\mathrm{GL}_{2}\left(\mathbb{F}_{q}\right)$ ) either in the Borel subgroup of $\mathrm{GL}_{2}\left(\mathbb{F}_{q}\right)$, or in the non-split torus of $\mathrm{GL}_{2}\left(\mathbb{F}_{q}\right)$. The second case cannot occur if $f$ is ordinary at $p$.

(ii) $\operatorname{Ind}_{F}^{\mathbb{Q}} \bar{\rho}\left(I_{p}\right) \subset \phi\left(H\left(\mathbb{F}_{q}\right)\right)$.

Proof. - (i) By Corollary $\left.2.13 \bar{\rho}\right|_{I_{\mathfrak{p}}} ^{\text {s.s. }}=\varepsilon_{\mathfrak{p}} \oplus \delta_{\mathfrak{p}}$, where $\varepsilon_{\mathfrak{p}}, \delta_{\mathfrak{p}}: I_{\mathfrak{p}} \rightarrow \overline{\mathbb{F}}_{p}^{\times}$are two tame characters of level $h:=\left|J_{F, \mathfrak{p}}\right|$ or $2\left|J_{F, \mathfrak{p}}\right|$ whose product equals $\omega^{1-k_{0}}$ and whose sum has FontaineLaffaille weights $\left(m_{\tau}, k_{0}-m_{\tau}-1\right)_{\tau \in J_{F, p}}$.

Let $x_{h}$ be a generator of $\mathbb{F}_{p^{h}}^{\times}$, and let $\varepsilon$ and $\delta$ be the characters of $\mathbb{F}_{p^{h}}^{\times}$deduced from $\varepsilon_{\mathfrak{p}}$ and $\delta_{\mathfrak{p}}$. Since by $\left(\mathbf{L I}_{\bar{\rho}}\right)$ the traces of the elements of $\bar{\rho}\left(\mathcal{G}_{\widehat{F}}\right)$ are in $F_{q} \amalg \theta \mathbb{F}_{q}$ (see Section 3.4), we deduce that $\left(\varepsilon\left(x_{h}\right)+\delta\left(x_{h}\right)\right)^{2} \in \mathbb{F}_{q}$ and hence $\varepsilon\left(x_{h}\right)^{2}+\delta\left(x_{h}\right)^{2} \in \mathbb{F}_{q}$.

If $\varepsilon\left(x_{h}\right)^{2}, \delta\left(x_{h}\right)^{2} \in \mathbb{F}_{q}^{\times}$, then it is easy to see that $\varepsilon\left(x_{h}\right), \delta\left(x_{h}\right) \in \mathbb{F}_{q}^{\times}$(we use $p>k_{0}$ and $\left.p \neq 2 k_{\tau}-1\right)$. In this case $I_{\mathfrak{p}}$ fixes a $\mathbb{F}_{q}$-rational line and therefore $\bar{\rho}\left(I_{\mathfrak{p}}\right)$ is contained in a Borel subgroup of $\mathrm{GL}_{2}\left(\mathbb{F}_{q}\right)$.

Otherwise $\varepsilon\left(x_{h}\right)^{2}$ and $\delta\left(x_{h}\right)^{2}$ are conjugated by the non-trivial element of $\operatorname{Gal}\left(\mathbb{F}_{q^{2}} / \mathbb{F}_{q}\right)$, hence $\varepsilon\left(x_{h}\right)^{2}=\delta\left(x_{h}\right)^{2 q}$. Since $p>2 k_{0}$, we have $\varepsilon\left(x_{h}\right)=\delta\left(x_{h}\right)^{q}$ and so $\varepsilon\left(x_{h}\right)+\delta\left(x_{h}\right)^{q} \in \mathbb{F}_{q}^{\times}$. Hence $\operatorname{tr}\left(\bar{\rho}\left(I_{\mathfrak{p}}\right)\right) \subset \mathbb{F}_{q}$ and $\bar{\rho}\left(I_{\mathfrak{p}}\right) \subset \mathrm{GL}_{2}\left(\mathbb{F}_{q}\right)$. In this case $\bar{\rho}\left(I_{\mathfrak{p}}\right)$ is contained in a non-split torus of $\mathrm{GL}_{2}\left(\mathbb{F}_{q}\right)$. If $f$ is ordinary at $p$, then the Fontaine-Laffaille weights of $\delta_{\mathfrak{p}}$ are strictly smaller than those of $\varepsilon_{\mathfrak{p}}$, and therefore the second case cannot occur.

(ii) The determinant condition ${ }^{\mathcal{D}}$ being satisfied, all we have to check is the following: for all $i \in I$ and $\tau, \tau^{\prime} \in J_{F}^{i}$ the character

$$
I_{p} \rightarrow\{ \pm 1\}, \quad y \mapsto\left(\bar{\rho}\left(\tilde{\sigma}_{i, \tau}^{-1} y \tilde{\sigma}_{i, \tau}\right)\right)^{-1}\left(\bar{\rho}\left(\tilde{\sigma}_{i, \tau^{\prime}}^{-1} y \tilde{\sigma}_{i, \tau^{\prime}}\right)\right)
$$

is trivial. This follows, as in the proof of Proposition 3.13, from the fact that $p>2 k_{0}$.

LEMmA 3.16. - Assume that $\left(\mathbf{L I}_{\bar{\rho}}\right)$ holds. Then $\phi\left(H\left(\mathbb{F}_{q}\right)\right) \subset \operatorname{Ind}_{F}^{\mathbb{Q}} \bar{\rho}\left(\mathcal{G}_{\widehat{F}}\right)$. 
Proof. - We have seen in the beginning of this paragraph that

$$
\operatorname{pr}\left(\phi\left(\mathrm{SL}_{2}\left(\mathbb{F}_{q}\right)^{I}\right)\right) \subset \operatorname{pr}\left(\operatorname{Ind}_{F}^{\mathbb{Q}} \bar{\rho}\left(\mathcal{G}_{\widehat{F}}\right)\right) .
$$

By Lemma 3.10, we deduce that $\phi\left(\mathrm{PSL}_{2}\left(\mathbb{F}_{q}\right)^{I}\right) \subset \operatorname{Ind}_{F}^{\mathbb{Q}} \bar{\rho}\left(\mathcal{G}_{\widehat{F}}\right)$.

Since $\phi\left(H\left(\mathbb{F}_{q}\right)\right)=\phi\left(\mathrm{SL}_{2}\left(\mathbb{F}_{q}\right)^{I}\right) \operatorname{Ind}_{F}^{\mathbb{Q}} \bar{\rho}\left(I_{p}\right)$, we are done.

Proposition 3.17. - Assume that $\left(\mathbf{L I}_{\bar{\rho}}\right)$ holds but $\left(\mathbf{L I}_{\text {Ind } \bar{\rho}}\right)$ fails for some $p>2 k_{0}$ (respectively for infinitely many primes $p$ ). Then, there exist $\tau \in J_{F}, \tau \neq \operatorname{id}_{F}$ and a finite order Hecke character $\varepsilon$ of $\widetilde{F}$ of conductor dividing $\mathrm{N}_{F / \mathbb{Q}}(\mathfrak{n})$ such that for all primes $v$ of $\widetilde{F}$ not dividing $\mathrm{N}_{F / \mathbb{Q}}(\mathfrak{n}) p$ we have $c\left(f_{\tau}, v\right) \equiv \varepsilon(v) c(f, v)(\bmod \mathcal{P})$ (respectively $c\left(f_{\tau}, v\right)=$ $\varepsilon(v) c(f, v))$.

Proof. - Since $\left(\mathbf{L I}_{\bar{\rho}}\right)$ holds but $\left(\mathbf{L I}_{\text {Ind } \bar{\rho}}\right)$ fails, there exist $\tilde{\tau}_{1}, \tilde{\tau}_{2} \in \mathcal{G}_{\mathbb{Q}}$ such that

$$
\tau:=\left.\tilde{\tau}_{2}^{-1} \tilde{\tau}_{1}\right|_{F} \neq \operatorname{id}_{F}
$$

and such that $\operatorname{pr} \bar{\rho}\left(\tilde{\tau}_{2}^{-1} y \tilde{\tau}_{1}\right)=\operatorname{pr} \bar{\rho}\left(\tilde{\tau}_{2}^{-1} y \tilde{\tau}_{2}\right)$, for all $y \in \mathcal{G}_{\widehat{F}}$. Since $\mathcal{G}_{\widehat{F}}$ is a normal subgroup of $\mathcal{G}_{\widetilde{F}}$, the above relation holds for every $y \in \mathcal{G}_{\widetilde{F}}$. Therefore, there exists a character $\varepsilon_{\text {gal }}: \mathcal{G}_{\widetilde{F}} \rightarrow \kappa^{\times}$such that for all $y \in \mathcal{G}_{\widetilde{F}}, \bar{\rho}_{f_{\tau}}(y)=\varepsilon_{\text {gal }}(y) \bar{\rho}_{f}(y)$. Since $p>2 k_{0}$, the same argument as in the proof of Proposition 3.13 shows that $\varepsilon_{\text {gal }}$ is unramified at primes dividing $p$. By Lemma $3.3, \varepsilon_{\text {gal }}$ can then be lifted to a finite order Hecke character $\varepsilon$ of $\widetilde{F}$ of conductor dividing $\mathrm{N}_{F / \mathbb{Q}}(\mathfrak{n})$. By evaluating at Frob ${ }_{v}$, for every prime $v \nmid \mathrm{N}_{F / \mathbb{Q}}(\mathfrak{n}) p$ of $\widetilde{F}$, we obtain

$$
c\left(f_{\tau}, v\right) \equiv \varepsilon(v) c(f, v)(\bmod \mathcal{P}) .
$$

By the determinant relation $\overline{\psi_{\tau}}=\varepsilon_{\text {gal }}^{2} \bar{\psi}$, there are finitely many such characters $\varepsilon$. Therefore, if $\left(\mathbf{L} \mathbf{I}_{\text {Ind } \bar{\rho}}\right)$ fails for infinitely many primes $p$, then the above congruence will be an equality.

COROLLARY 3.18. - Assume that $F$ is a Galois field of odd degree and the central character $\psi$ of $f$ is trivial $(F=\widehat{F})$. Assume moreover that $f$ is not a theta series and that $\left(\mathbf{L I}_{\text {Ind } \bar{\rho}}\right)$ does not hold for infinitely many primes $p$. Then, there exist a subfield $F^{\prime} \subsetneq F$ and a Hilbert modular form $f^{\prime}$ on $F^{\prime}$, such that the base change of $f^{\prime}$ to $F$ is a twist of $f$ by a quadratic character of conductor dividing $\mathrm{N}_{F / \mathbb{Q}}(\mathfrak{n})$.

Proof. - As in the proof of Proposition 3.17 there exist a quadratic character $\varepsilon$ of $F$ of conductor dividing $\mathrm{N}_{F / \mathbb{Q}}(\mathfrak{n})$ and $\operatorname{id}_{F} \neq \tau \in \operatorname{Gal}(F / \mathbb{Q})$ such that $\rho_{f_{\tau}}=\varepsilon_{\text {gal }} \otimes \rho=\rho_{f \otimes \varepsilon}$. Let $F^{\prime} \subset F$ (respectively $F_{i} \supset F$ ) be the fixed field of $\tau$ (respectively of $\operatorname{ker}\left(\varepsilon_{\tau^{i}}\right)$ ). We know that $F / F^{\prime}$ is a cyclic extension of $o d d$ degree $h$. Let $F^{\prime \prime}=\prod_{i=1}^{h} F_{i}$. Then we have

$$
\operatorname{Gal}\left(F^{\prime \prime} / F^{\prime}\right)=\left\{\left(u_{1}, \ldots, u_{h}\right) \in\{ \pm 1\}^{h} \mid \prod_{i=1}^{h} u_{i}=1\right\} \rtimes\left\{\tau^{i} \mid 0 \leqslant i \leqslant h-1\right\},
$$

where $\tau$ acts on $\left(u_{1}, \ldots, u_{h}\right)$ by cyclic permutation. When $h=3$ the group $\operatorname{Gal}\left(F^{\prime \prime} / F^{\prime}\right)$ is isomorphic to $A_{4}$.

The representation $\left.\rho\right|_{\mathcal{G}_{F^{\prime \prime}}}$ is invariant by $\operatorname{Gal}\left(F^{\prime \prime} / F^{\prime}\right)$, but Langlands Cyclic Descent does not apply directly because the order of $\operatorname{Gal}\left(F^{\prime \prime} / F^{\prime}\right)$ is even. Consider the quadratic character $\delta=\varepsilon \cdot \varepsilon_{\tau^{2}} \cdots \varepsilon_{\tau^{h-1}}$. Then the $\mathcal{G}_{F}$-representation $\delta_{\text {gal }} \otimes \rho$ is invariant by the group $\operatorname{Gal}\left(F / F^{\prime}\right)$, so extends to a representation of $\mathcal{G}_{F^{\prime}}$. By applying Langlands Cyclic Descent to $\delta \otimes f$ we obtain $f^{\prime}$ as desired.

$4^{\mathrm{e}}$ SÉRIE - TOME $38-2005-\mathrm{N}^{\circ} 4$ 


\section{Boundary cohomology and congruence criterion}

We recall that $f \in S_{k}(\mathfrak{n}, \psi)$ is supposed to be a Hilbert modular newform.

Definition 4.1. - We say that a normalized eigenform $g \in S_{k}(\mathfrak{n}, \psi)$ is congruent to $f$ modulo $\mathcal{P}$ if their respective eigenvalues for the Hecke operators (that is their Fourier coefficients) are congruent modulo $\mathcal{P}$.

We say that a prime $\mathcal{P}$ is a congruence prime for $f$ if there exists a normalized eigenform $g \in S_{k}(\mathfrak{n}, \psi)$ distinct from $f$ and congruent to $f$ modulo $\mathcal{P}$.

One expects that, as in the elliptic modular case (carried out by Hida [21,22] and Ribet [36]), the congruence primes for $f$ are controlled by the value at 1 of the adjoint $L$-function of $f$. Such results have been obtained by Ghate [18] when $k$ is parallel.

Following [21,18] and using a vanishing result of the boundary cohomology of a Hilbert modular variety we obtain a new result in this direction (see Theorems 4.11 and 6.7(ii)).

\subsection{Vanishing of certain local components of the boundary cohomology}

We introduce the following condition:

(MW) the middle weight $\frac{\left|p\left(J_{F}\right)\right|+|p(\emptyset)|}{2}=\frac{d\left(k_{0}-1\right)}{2}$ does not belong to $\left\{|p(J)|, J \subset J_{F}\right\}$.

This condition is automatically satisfied when the motivic weight $d\left(k_{0}-1\right)$ is odd, or when $d=2$ and $k$ is non-parallel.

LEMMA 4.2. - Let $\rho_{0}$ be a representation of $\mathcal{G}_{\widetilde{F}}$ on a finite-dimensional $\kappa$-vector space $W$. Assume that for every y $\in \mathcal{G}_{\widetilde{F}}$, the characteristic polynomial of $\left(\otimes \operatorname{Ind}_{F}^{\mathbb{Q}} \bar{\rho}\right)(y)$ annihilates $\rho_{0}(y)$.

(i) If (I), (II) and $\left(\mathbf{L I}_{\bar{\rho}}\right)$ hold, then for all $h \in \mathbb{Z}$ the weights $h$ and $d\left(k_{0}-1\right)-h$ occur with the same multiplicity in each $\mathcal{G}_{\widetilde{F}}$-irreducible subquotient of $\rho_{0}$.

(ii) If (I), $\left(\mathbf{I r r}_{\bar{\rho}}\right)$ and (MW) hold and $p-1>\max \left(1, \frac{5}{d}\right) \sum_{\tau \in J_{F}}\left(k_{\tau}-1\right)$, then each $\mathcal{G}_{\widetilde{F}^{-}}$ irreducible subquotient of $\rho_{0}$ contains at least two different weights for the action of the tame inertia at $p$.

Proof. - We may assume that $\rho_{0}$ is irreducible.

(i) By Lemmas 3.15(ii) and 3.16 we have $\operatorname{Ind}_{F}^{\mathbb{Q}} \bar{\rho}\left(I_{p}\right) \subset \phi\left(H\left(\mathbb{F}_{q}\right)\right) \subset \operatorname{Ind}_{F}^{\mathbb{Q}} \bar{\rho}\left(\mathcal{G}_{\widehat{F}}\right)$. Let $T^{\prime}$ be the torus of $H\left(\mathbb{F}_{q}\right)$ containing the image of the tame inertia, and $N^{\prime}$ be the normalizer of $T^{\prime}$ in $H\left(\mathbb{F}_{q}\right)$. The image of $N^{\prime} / T^{\prime} \cong\{ \pm 1\}^{I}$ by $\phi$ is the subgroup of the Weyl group $N / T=\{ \pm 1\}^{J_{F}}$ of $G$ containing the elements which are constant on the partition $J_{F}=\bigsqcup_{i \in I} J_{F}^{i}$. In particular, the longest Weyl element $\epsilon_{J_{F}}$ belongs to the image of $N^{\prime} / T^{\prime}$.

Let $x \in W$ be an eigenvector for the action of $T^{\prime}$. By the annihilation condition, there exists a subset $J_{x} \subset J_{F}$, such that $I_{p}$ acts on $x$ by the weight $\left|p\left(J_{x}\right)\right|$.

Let $y_{J_{F}} \in \mathcal{G}_{\widetilde{F}}$ be such that $\operatorname{Ind}_{F}^{\mathbb{Q}} \bar{\rho}\left(y_{J_{F}}\right)=\epsilon_{J_{F}} \bmod T^{\prime}$. Then $\rho_{0}\left(y_{J_{F}}\right)(x)$ is of weight $\left|p\left(J_{x} \Delta J_{F}\right)\right|=\hat{d}\left(k_{0}-1\right)-\left|p\left(J_{x}\right)\right|$. Therefore, for each $h \in \mathbb{Z}, \rho_{0}\left(y_{J_{F}}\right)$ gives a bijection between the eigenspaces for the tame inertia of weight $h$ and $d\left(k_{0}-1\right)-h$.

(ii) If $\left(\mathbf{L I}_{\bar{\rho}}\right)$ holds, then the statement follows from (i) and (MW). Otherwise, by Proposition 3.8 the group $\operatorname{pr}\left(\bar{\rho}\left(\mathcal{G}_{F}\right)\right)$ is dihedral. Since $\widetilde{F}$ is totally real, $\operatorname{pr}\left(\bar{\rho}\left(\mathcal{G}_{\widetilde{F}}\right)\right)$ is also dihedral (see Section 3.3).

Denote by $N$ the normalizer of the standard torus $T$ in $G$. Put $N^{\prime}=\operatorname{Ind}_{F}^{\mathbb{Q}} \bar{\rho}\left(\mathcal{G}_{\widetilde{F}}\right) \subset N(\kappa)$ and $T^{\prime}=N^{\prime} \cap T(\kappa)$. Then $N^{\prime} / T^{\prime}$ is a subgroup of the Weyl group $\{ \pm 1\}^{J_{F}}=N / T$ of $G$.

As we have seen in Section 3.3, the representation $\operatorname{Ind}_{F}^{\mathbb{Q}} \bar{\rho}$ is tamely ramified at $p$ and the image of the inertia group $I_{p}$ is contained in $T^{\prime}$.

Let $x \in W$ be an eigenvector for the action of $T^{\prime}$. By the annihilation condition, there exists a subset $J_{x} \subset J_{F}$, such that $I_{p}$ acts on $x$ by the weight $\left|p\left(J_{x}\right)\right|$. For every element $\epsilon_{J} \in N^{\prime} / T^{\prime}$, 
$\left(J \subset J_{F}\right)$, let $y_{J} \in \mathcal{G}_{\widetilde{F}}$ be such that $\operatorname{Ind}_{F}^{\mathbb{Q}} \bar{\rho}\left(y_{J}\right)=\epsilon_{J} \bmod T^{\prime}$. Then $\rho_{0}\left(y_{J}\right)(x)$ is of weight $\left|p\left(J_{x} \Delta J\right)\right|$. It remains to show that the $\left|p\left(J_{x} \Delta J\right)\right|$ are not all equal when $\epsilon_{J}$ runs over the elements of $N^{\prime} / T^{\prime}$. Note that, for all $\tau \in J_{F}$, the $\tau$-projection $N^{\prime} / T^{\prime} \rightarrow\{ \pm 1\}$ is a surjective homomorphism (because the group $\operatorname{pr}\left(\bar{\rho}_{f_{\tau}}\left(\mathcal{G}_{\widetilde{F}}\right)\right)$ is also dihedral). Therefore, we have:

$$
\sum_{\epsilon_{J} \in N^{\prime} / T^{\prime}}\left|p\left(J_{x} \Delta J\right)\right|=\left|N^{\prime} / T^{\prime}\right| \frac{d\left(k_{0}-1\right)}{2} .
$$

The statement now follows from the (MW) assumption.

Remark 4.3. - The first part of the previous lemma is a generalization from the quadratic to the arbitrary degree case of the key lemma in [8]. This lemma is false in general under the only assumptions (I), (II) and $\left(\operatorname{Irr}_{\bar{\rho}}\right)$ when $d \geqslant 3$. In fact, consider the following construction in the cubic case: let $L$ be a Galois extension of $\mathbb{Q}$ of group $A_{4}$, such that the cubic subfield $F$ fixed by the Klein group is totally real; let $K$ be a quadratic extension of $F$ in $L$ and consider a theta series $f$ of weight $(2,2,2)$ attached to a Hecke character of $K$; then the tensor induced representation $\otimes \operatorname{Ind}_{F}^{\mathbb{Q}} \rho$ has two irreducible four-dimensional subquotients of Hodge-Tate weights $(0,2,2,2)$ and $(1,1,1,3)$.

As in the introduction, let $\mathbb{T}^{\prime} \subset \mathbb{T}$ denote the subalgebra generated by the Hecke operators outside a finite set of places containing those dividing $\mathfrak{n} p$.

THEOREM 4.4. - Assume that (I), $\left(\mathbf{I r r}_{\bar{\rho}}\right)$ and $(\mathbf{M W})$ hold, and

$$
p-1>\max \left(1, \frac{5}{d}\right) \sum_{\tau \in J_{F}}\left(k_{\tau}-1\right)
$$

Denote by $\mathfrak{m}$ the maximal ideal of $\mathbb{T}$ corresponding to $f$ and $\iota_{p}$ and put $\mathfrak{m}^{\prime}=\mathfrak{m} \cap \mathbb{T}^{\prime}$. Then

(i) the $\mathfrak{m}^{\prime}$-torsion of the boundary cohomology $\mathrm{H}_{\partial}^{\bullet}\left(Y, \mathbb{V}_{n}(\kappa)\right)\left[\mathfrak{m}^{\prime}\right]$ vanishes,

(ii) the Poincaré pairing $\mathrm{H}_{!}^{d}\left(Y, \mathbb{V}_{n}(\mathcal{O})\right)_{\mathfrak{m}^{\prime}}^{\prime} \times \mathrm{H}_{!}^{d}\left(Y, \mathbb{V}_{n}(\mathcal{O})\right)_{\mathfrak{m}^{\prime}}^{\prime} \rightarrow \mathcal{O}$ is a perfect duality of free $\mathcal{O}$-modules of finite rank,

(iii) $\mathrm{H}^{\bullet}\left(Y, \mathbb{V}_{n}(\mathcal{O})\right)_{\mathfrak{m}^{\prime}}=\mathrm{H}_{c}^{\bullet}\left(Y, \mathbb{V}_{n}(\mathcal{O})\right)_{\mathfrak{m}^{\prime}}=\mathrm{H}_{!}^{\bullet}\left(Y, \mathbb{V}_{n}(\mathcal{O})\right)_{\mathfrak{m}^{\prime}}$

Proof. - (i) Consider the minimal compactification $Y_{\overline{\mathbb{Q}}} \stackrel{j}{\hookrightarrow} Y_{\overline{\mathbb{Q}}}^{*} \stackrel{i}{\hookrightarrow} \partial Y_{\overline{\mathbb{Q}}}^{*}$. The Hecke correspondences extend to $Y_{\mathbb{Q}}^{*}$. By the Betti-étale comparison isomorphism, we identify (in a Heckeequivariant way) the following two long exact cohomology sequences:

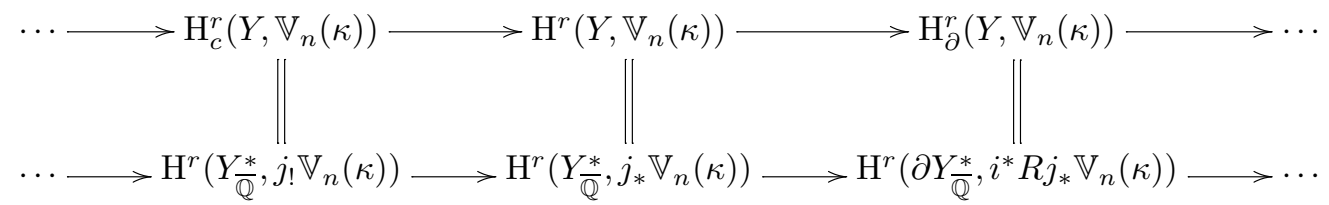

Consider the $\mathcal{G}_{\mathbb{Q}}$-module $W_{\partial}^{r}=\mathrm{H}^{r}\left(\partial Y_{\overline{\mathbb{Q}}}^{*}, i^{*} R j_{*} \mathbb{V}_{n}(\kappa)\right)$. We have to show that $W_{\partial}^{r}\left[\mathfrak{m}^{\prime}\right]=0$.

By the Cebotarev Density Theorem and the congruence relations at totally split primes of $F$, we can apply Lemma 4.2 to $W_{\partial}^{r}\left[\mathfrak{m}^{\prime}\right]$. Therefore each $\mathcal{G}_{\widehat{F}}$-irreducible subquotient of $W_{\partial}^{r}\left[\mathfrak{m}^{\prime}\right]$ has at least two different weights for the action of the tame inertia at $p$. So it is enough to show that each $\mathcal{G}_{\mathbb{Q}}$-irreducible subquotient of $W_{\partial}^{r}$ is pure (= contains a single weight for the action of the 
tame inertia at $p$ ). Since $\partial Y_{\mathbb{Q}}^{*}$ is zero-dimensional, the spectral sequence

$$
\mathrm{H}^{\bullet}\left(\partial Y_{\overline{\mathbb{Q}}}^{*}, i^{*} R^{\bullet} j_{*} \mathbb{V}_{n}(\kappa)\right) \Longrightarrow \mathrm{H}^{\bullet}\left(\partial Y_{\overline{\mathbb{Q}}}^{*}, i^{*} R j_{*} \mathbb{V}_{n}(\kappa)\right)
$$

shows that $W_{\partial}^{r}=\mathrm{H}^{0}\left(\partial Y_{\overline{\mathbb{Q}}}^{*}, i^{*} R^{r} j_{*} \mathbb{V}_{n}(\kappa)\right)$.

Since $\mathrm{H}^{0}\left(\partial Y_{\overline{\mathbb{Q}}}^{*}, i^{*} R^{r} j_{*} \mathbb{V}_{n}(\kappa)\right)$ is a subquotient of $\mathrm{H}^{0}\left(\partial Y_{\overline{\mathbb{Q}}}^{1, *}, i^{*} R^{r} j_{*} \mathbb{V}_{n}(\kappa)\right)$ it is enough to show that each $\mathcal{G}_{\mathbb{Q}}$-irreducible subquotient of this last is pure.

This will be done using a result of Pink [32]. We replaced $Y$ by $Y^{1}$, since the group $G$ does not satisfy the conditions of this reference, while $G^{*}$ satisfies them.

Consider the decomposition $T=D_{l} \times D_{h}$, according to

$$
\left(\begin{array}{cc}
u \epsilon & 0 \\
0 & u^{-1}
\end{array}\right)=\left(\begin{array}{cc}
u & 0 \\
0 & u^{-1}
\end{array}\right)\left(\begin{array}{cc}
\epsilon & 0 \\
0 & 1
\end{array}\right) .
$$

Put $\Gamma^{1}=\Gamma_{1}^{1}(\mathfrak{c}, \mathfrak{n})$. By [32, Theorem 5.3.1], the restriction of the étale sheaf $i^{*} R^{r} j_{*} \mathbb{V}_{n}\left(\mathbb{F}_{p}\right)$ to a $\operatorname{cusp} \mathcal{C}=\gamma \infty$ of $Y_{\overline{\mathbb{Q}}}^{1, *}$ is the image by the functor of Pink of the $\gamma^{-1} \Gamma^{1} \gamma \cap B / \gamma^{-1} \Gamma^{1} \gamma \cap D_{l} U$ module

$$
\bigoplus_{a+b=r} \mathrm{H}^{a}\left(\gamma^{-1} \Gamma^{1} \gamma \cap D_{l}, \mathrm{H}^{b}\left(\gamma^{-1} \Gamma^{1} \gamma \cap U, \mathbb{V}_{n}\left(\mathbb{F}_{p}\right)\right)\right) .
$$

Under the assumption (II), a modulo $p$ version of a theorem of Kostant (see [33]) gives an isomorphism of $T$-module $\mathrm{H}^{b}\left(\gamma^{-1} \Gamma^{1} \gamma \cap U, \mathbb{V}_{n}\left(\mathbb{F}_{p}\right)\right)=\bigoplus_{|J|=b} W_{\epsilon_{J}(n+t)-t, n_{0}}$. By decomposing $W_{\epsilon_{J}(n+t)-t}=W_{\epsilon_{J}(n+t)-t, n_{0}}^{l} \otimes W_{\epsilon_{J}(n+t)-t, n_{0}}^{h}$ according to $T=D_{l} \times D_{h}$, we get

$$
\begin{aligned}
& \mathrm{H}^{a}\left(\gamma^{-1} \Gamma^{1} \gamma \cap D_{l}, \mathrm{H}^{b}\left(\gamma^{-1} \Gamma^{1} \gamma \cap U, \mathbb{V}_{n}\left(\mathbb{F}_{p}\right)\right)\right) \\
& \quad=\bigoplus_{|J|=b} \mathrm{H}^{a}\left(\gamma^{-1} \Gamma^{1} \gamma \cap D_{l}, W_{\epsilon_{J}}^{l}(n+t)-t, n_{0}\right) \otimes W_{\epsilon_{J}(n+t)-t, n_{0}}^{h},
\end{aligned}
$$

where Galois acts only on the second factors of the right-hand side.

Therefore $\mathrm{H}^{0}\left(\partial Y_{\mathbb{Q}}^{*}, i^{*} R^{r} j_{*} \mathbb{V}_{n}\left(\mathbb{F}_{p}\right)\right)$ is a direct sum of subspaces $\mathrm{H}^{0}\left(\mathcal{C}, W_{\epsilon_{J}(n+t)-t, n_{0}}^{h}\left(\mathbb{F}_{p}\right)\right)$, $|J| \leqslant r$, each containing a single Fontaine-Laffaille weight, namely the weight $|p(J)|$.

(ii) Since the Poincaré duality is perfect over $E$, it is enough to show that the $\mathfrak{m}^{\prime}$-localization of natural map $\mathrm{H}^{d}(\mathcal{O}) / \mathrm{H}_{!}^{d}(\mathcal{O}) \rightarrow \mathrm{H}^{d}(E) / \mathrm{H}_{!}^{d}(E)$ is injective. For this, it is sufficient to show that $\mathrm{H}_{\partial}^{d}(\mathcal{O})_{\mathfrak{m}^{\prime}}:=\mathrm{H}^{d}\left(\partial M, \mathbb{V}_{n}(\mathcal{O})\right)_{\mathfrak{m}^{\prime}}$ is torsion free, which would follow from the vanishing of $\mathrm{H}_{\partial}^{d-1}(E / \mathcal{O})_{\mathfrak{m}^{\prime}}$. We have a surjection $\mathrm{H}_{\partial}^{d-1}(\kappa)_{\mathfrak{m}^{\prime}} \rightarrow \mathrm{H}_{\partial}^{d-1}(E / \mathcal{O})_{\mathfrak{m}^{\prime}}[\varpi]$, where $\varpi$ is an uniformizer of $\mathcal{O}$. Finally, by (i) and Nakayama's lemma, $\mathrm{H}_{\partial}^{d-1}(\kappa)_{\mathfrak{m}^{\prime}}=0$.

(iii) The vanishing of $\mathrm{H}_{\partial}^{\bullet}(\kappa)_{\mathfrak{m}^{\prime}}$ gives the vanishing of $\mathrm{H}_{\partial}^{\bullet}(\mathcal{O})_{\mathfrak{m}^{\prime}}=0$.

\subsection{Definition of periods}

By taking the subspace $\bigcap_{\mathfrak{a} \subset \mathfrak{o}} \operatorname{ker}\left(T_{\mathfrak{a}}-c(f, \mathfrak{a})\right)$ of (8) we obtain

$$
\delta_{J}: \mathbb{C} f_{J} \stackrel{\sim}{\longrightarrow} \mathrm{H}_{!}^{d}\left(Y^{\mathrm{an}}, \mathbb{V}_{n}(\mathbb{C})\right)\left[\hat{\epsilon}_{J}, f\right] .
$$

Fix an isomorphism $\mathbb{C} \cong \overline{\mathbb{Q}}_{p}$ compatible with $\iota_{p}$. We recall that $\mathrm{H}_{!}^{d}\left(Y^{\text {an }}, \mathbb{V}_{n}(\mathcal{O})\right)^{\prime}$ denotes the image of the natural map $\mathrm{H}_{c}^{d}\left(Y^{\text {an }}, \mathbb{V}_{n}(\mathcal{O})\right) \rightarrow \mathrm{H}^{d}\left(Y^{\text {an }}, \mathbb{V}_{n}(\mathbb{C})\right)$. Since $\mathcal{O}$ is principal, the $\mathcal{O}$-module $L_{f, J}:=\mathrm{H}_{!}^{d}\left(Y^{\text {an }}, \mathbb{V}_{n}(\mathcal{O})\right)^{\prime}\left[\hat{\epsilon}_{J}, f\right]$ is free of rank 1 . We fix a basis $\eta(f, J)$ of $L_{f, J}$. 
DEFinition 4.5. - For each $J \subset J_{F}$ we define the period $\Omega(f, J)=\frac{\delta_{J}\left(f_{J}\right)}{\eta(f, J)} \in \mathbb{C}^{\times} / \mathcal{O}^{\times}$. We fix $J_{0} \subset J_{F}$ and put $\Omega_{f}^{+}=\Omega\left(f, J_{0}\right)$ and $\Omega_{f}^{-}=\Omega\left(f, J_{F} \backslash J_{0}\right)$.

Remark 4.6. - The periods $\Omega_{f}^{ \pm}$differ from the ones originally introduced by Hida in [21]. Hida's periods put together all the external conjugates of $f$. Our slightly different definition is motivated by the congruence criterion that we want to show (Theorem 4.11). Since we can prove the perfectness of the twisted Poincare pairing only for certain local components of the middle degree cohomology $\mathrm{H}_{!}^{d}\left(Y^{\text {an }}, \mathbb{V}_{n}(\mathcal{O})\right)^{\prime}$ and in general $f$ and its external conjugates do not belong to the same local component, we have to separate them in the definition of the period.

\subsection{Computation of a discriminant}

The aim of this paragraph is to compute the discriminant $\operatorname{disc}\left(L_{f}\right)$ of the $\mathcal{O}$-lattice $L_{f}:=\mathrm{H}_{!}^{d}\left(Y^{\mathrm{an}}, \mathbb{V}_{n}(\mathcal{O})\right)^{\prime}[f]=\bigoplus_{J \subset J_{F}} L_{f, J}$, with respect to the twisted Poincare pairing [,] defined in (6). We follow [18, Section 6].

We have $\operatorname{disc}\left(L_{f}\right)=\operatorname{det}\left(\left(\left[\eta(f, J), \eta\left(f, J^{\prime}\right)\right]\right)_{J, J^{\prime} \subset J_{F}}\right)$.

By $[18,(41)]$, for every $\tau \in J_{F}$ and $x, y \in \mathrm{H}_{!}^{d}\left(Y^{\mathrm{an}}, \mathbb{V}_{n}(\mathbb{C})\right)$ we have $\left[\epsilon_{\tau} \cdot x, y\right]=-\left[x, \epsilon_{\tau} \cdot y\right]$.

The embedding $\mathcal{O} \hookrightarrow \mathbb{C}$ that we have fixed gives an embedding $\tau_{0}: F \hookrightarrow \mathbb{C}$.

$$
\begin{aligned}
\operatorname{disc}\left(L_{f}\right) & =\prod_{\tau_{0} \in J \subset J_{F}}\left|\begin{array}{cc}
0 & {\left[\eta(f, J), \eta\left(f, J_{F} \backslash J\right)\right]} \\
0
\end{array}\right| \\
& =\prod_{\tau_{0} \in J \subset J_{F}}-\left(\frac{\left[\delta_{J}(f), \delta_{J_{F} \backslash J}(f)\right]}{\Omega(f, J) \Omega\left(f, J_{F} \backslash J\right)}\right)^{2},
\end{aligned}
$$

and $\left[\delta_{J}(f), \delta_{J_{F} \backslash J}(f)\right]=2^{d}\left\langle\epsilon_{J_{F}} \delta(f), \iota \cdot \delta(f)\right\rangle=2^{d} W(f)\left\langle\epsilon_{J_{F}} \delta(f), \delta\left(f^{c}\right)\right\rangle=2^{d} W(f)(f, f)_{K_{1}(\mathfrak{n})}$, where $f^{c}$ is the complex conjugate of $f$ and $W(f)$ is the complex constant of the functional equation of the standard $L$-function of $f$. By [9, Lemma 2.13] $W(f) \in \mathcal{O}^{\times}$. Therefore the following equality holds in $E^{\times} / \mathcal{O}^{\times}$:

$$
\operatorname{disc}\left(L_{f, J_{0}} \oplus L_{f, J_{F} \backslash J_{0}}\right)=\left(\frac{(f, f)_{K_{1}(\mathfrak{n})}}{\Omega_{f}^{+} \Omega_{f}^{-}}\right)^{2} .
$$

4.4. Shimura's formula for $L\left(\operatorname{Ad}^{0}(f), 1\right)$

For a prime $v$ of $F$ we define $\alpha_{v}$ and $\beta_{v}$ by:

$$
\alpha_{v}+\beta_{v}=c(f, v), \quad \alpha_{v} \beta_{v}= \begin{cases}\psi(v) \mathrm{N}_{F / \mathbb{Q}}(v), & \text { if } v \nmid \mathfrak{n}, \\ 0, & \text { if } v \mid \mathfrak{n} .\end{cases}
$$

The naive adjoint $L$-function of $f$ is defined by the Euler product:

$$
\begin{aligned}
L^{(\mathfrak{n})}\left(\operatorname{Ad}^{0}(f), s\right)= & \prod_{v \nmid \mathfrak{n}}\left[\left(1-\alpha_{v} \beta_{v}^{-1} \mathrm{~N}_{F / \mathbb{Q}}(v)^{-s}\right)\left(1-\mathrm{N}_{F / \mathbb{Q}}(v)^{-s}\right)\right. \\
& \left.\times\left(1-\beta_{v} \alpha_{v}^{-1} \mathrm{~N}_{F / \mathbb{Q}}(v)^{-s}\right)\right]^{-1} .
\end{aligned}
$$

In [38] Shimura studies the series $D\left(f, f^{c}, s\right)=\sum_{\mathfrak{a} \subset \mathfrak{o}} c(f, \mathfrak{a}) \overline{c(f, \mathfrak{a})} \mathrm{N}_{F / \mathbb{Q}}(\mathfrak{a})^{-s}$ and shows that it has the following Euler product (see [26, Lemma 7.2]):

$4^{\mathrm{e}}$ SÉRIE - TOME $38-2005-\mathrm{N}^{\circ} 4$ 


$$
\begin{aligned}
D\left(f, f^{c}, s\right)= & \prod_{v}\left(1-\alpha_{v} \beta_{v} \overline{\alpha_{v} \beta_{v}} \mathrm{~N}_{F / \mathbb{Q}}(v)^{-2 s}\right)\left(1-\alpha_{v} \overline{\alpha_{v}} \mathrm{~N}_{F / \mathbb{Q}}(v)^{-s}\right)^{-1} \\
& \times\left(1-\alpha_{v} \overline{\beta_{v}} \mathrm{~N}_{F / \mathbb{Q}}(v)^{-s}\right)^{-1}\left(1-\beta_{v} \overline{\alpha_{v}} \mathrm{~N}_{F / \mathbb{Q}}(v)^{-s}\right)^{-1} \\
& \times\left(1-\beta_{v} \overline{\beta_{v}} \mathrm{~N}_{F / \mathbb{Q}}(v)^{-s}\right)^{-1} .
\end{aligned}
$$

Using the fact that for all $v \nmid \mathfrak{n} \overline{c(f, v)}=\overline{\psi(v)} c(f, v)$ a direct computation gives:

$$
\zeta_{F}^{(\mathfrak{n})}(2 s) D^{(\mathfrak{n})}\left(f, f^{c}, s+k_{0}-1\right)=\zeta_{F}^{(\mathfrak{n})}(s) L^{(\mathfrak{n})}\left(\operatorname{Ad}^{0}(f), s\right),
$$

where $D^{(\mathfrak{n})}\left(f, f^{c}, s\right)$ is obtained from $D\left(f, f^{c}, s\right)$ by removing the Euler factors for $v \mid \mathfrak{n}$.

TheOREM 4.7 (Shimura [38, (2.31), Proposition 4.13]). - Let $f \in S_{k}(\mathfrak{n}, \psi)$ be a newform. Then

$$
\operatorname{Res}_{s=1} D\left(f, f^{c}, s+k_{0}-1\right)=2^{d-1}(4 \pi)^{|k|} \prod_{\tau \in J_{F}} \Gamma\left(k_{\tau}\right)^{-1} R_{F}\left[\mathfrak{o}_{+}^{\times}: \mathfrak{o}^{\times 2}\right] \mu^{-1}(f, f)_{K_{1}(\mathfrak{n})},
$$

where $\mu=\mu\left(\Gamma_{1}(\mathfrak{c}, \mathfrak{n}) \backslash \mathfrak{H}_{F}\right)=\frac{2 \mathrm{~N}_{F / \mathbb{Q}}(\mathfrak{d})^{3 / 2} \zeta_{F}(2)}{\pi^{d}\left[\mathfrak{o}_{+}^{\times}: \mathfrak{o}^{\times 2}\right]} \cdot \frac{\mathrm{N}_{F / \mathbb{Q}}(\mathfrak{n})^{2} \prod_{v \mid \mathfrak{n}}\left(1-\mathrm{N}_{F / \mathbb{Q}}(v)^{-2}\right)}{\left[\mathfrak{o}^{\times}: \mathfrak{o}_{\mathfrak{n}, 1}^{\times}\right]}$.

By a direct computation:

$$
\begin{aligned}
\zeta_{F}^{(\mathfrak{n})} & (2) \operatorname{Res}_{s=1} D\left(f, f^{c}, s+k_{0}-1\right) \\
= & \frac{\operatorname{Res}_{s=1} \zeta_{F}^{(\mathfrak{n})}(s)(4 \pi)^{|k|} \pi^{d}\left[\mathfrak{o}_{+}^{\times}: \mathfrak{o}^{\times 2}\right]\left[\mathfrak{o}^{\times}: \mathfrak{o}_{\mathfrak{n}, 1}^{\times}\right](f, f)_{K_{1}(\mathfrak{n})}}{2 \Delta h_{F}^{+} \mathrm{N}_{F / \mathbb{Q}}(\mathfrak{n}) \prod_{\tau \in J_{F}} \Gamma\left(k_{\tau}\right) \prod_{v \mid \mathfrak{n}}\left(1-\mathrm{N}_{F / \mathbb{Q}}(v)^{-1}\right)} .
\end{aligned}
$$

We define the imprimitive adjoint $L$-function $L^{*}\left(\operatorname{Ad}^{0}(f), s\right)$ by completing the naive adjoint $L$-function $L^{(\mathfrak{n})}\left(\operatorname{Ad}^{0}(f), s\right)$ defined in (16), in order to have the relation:

$$
L^{*}\left(\operatorname{Ad}^{0}(f), s\right) D^{(\mathfrak{n})}\left(f, f^{c}, s+k_{0}-1\right)=L^{(\mathfrak{n})}\left(\operatorname{Ad}^{0}(f), s\right) D\left(f, f^{c}, s+k_{0}-1\right) .
$$

By [26, (7.7)] we have $L^{*}\left(\operatorname{Ad}^{0}(f), s\right)=L^{(\mathfrak{n})}\left(\operatorname{Ad}^{0}(f), s\right) \prod_{v \mid \mathfrak{n}} L_{v}^{*}\left(\operatorname{Ad}^{0}(f), s\right)$, where for $v \mid \mathfrak{n}$

$$
L_{v}^{*}\left(\operatorname{Ad}^{0}(f), s\right)=\left\{\begin{array}{l}
\left(1-\mathrm{N}_{F / \mathbb{Q}}(v)^{-s}\right)^{-1}, \\
\left(1-\mathrm{N}_{F / \mathbb{Q}}(v)^{-s-1}\right)^{-1}, \quad \text { if } f \text { is a principal series and minimal at } v \\
1, \quad \text { otherwise. }
\end{array}\right.
$$

Following Deligne [6] we associate to $L^{*}\left(\operatorname{Ad}^{0}(f), s\right)$ an Euler factor:

$$
\Gamma\left(\operatorname{Ad}^{0}(f), s\right)=\prod_{\tau \in J_{F}} \pi^{-(s+1) / 2} \Gamma((s+1) / 2)(2 \pi)^{1-k_{\tau}-s} \Gamma\left(s+k_{\tau}-1\right) .
$$

Finally, by (17) and (18), there exists $a \in \mathbb{Z}$ such that:

$$
\Gamma\left(\operatorname{Ad}^{0}(f), 1\right) L^{*}\left(\operatorname{Ad}^{0}(f), 1\right)=\frac{2^{a}}{\Delta}(f, f)_{\mathfrak{n}} .
$$

Remark 4.8. - Consider the adjoint $L$-function $L\left(\operatorname{Ad}^{0}(\rho), s\right)$ attached to the three-dimensional $\mathcal{G}_{F}$-representation $\operatorname{Ad}^{0}(\rho)$ on trace zero $2 \times 2$ matrices. By compatibility between local and global Langlands correspondence $L\left(\operatorname{Ad}^{0}(\rho), s\right)$ is equal to the adjoint $L$-function $L\left(\operatorname{Ad}^{0}(f), s\right)$ associated to the automorphic representation attached to $f$. Nevertheless $L\left(\operatorname{Ad}^{0}(f), s\right)$ may differ from $L^{*}\left(\operatorname{Ad}^{0}(f), s\right)$ at some places $v$ dividing $\mathfrak{n}$ (see [26, (7.3c)]). 


\subsection{Construction of congruences}

LEMMA 4.9. - Let $V_{1}$ and $V_{2}$ be two finite-dimensional E-vector spaces and let $L$ be a $\mathcal{O}$-lattice in $V=V_{1} \oplus V_{2}$. For $j=1,2$, put $L_{j}=L \cap V_{j}$ and denote $L^{j}$ the projection of $L$ in $V_{j}$ following the above direct sum decomposition. Then:

(i) $L_{j} \subset L^{j}$ are two lattices of $V_{j}$, and $L_{j}$ is a direct factor in $L$.

(ii) we have isomorphisms of finite $\mathcal{O}$-modules:

$$
L^{1} / L_{1} \stackrel{\sim}{\sim} L / L_{1} \oplus L_{2} \stackrel{\sim}{\longrightarrow} L^{2} / L_{2}
$$

This finite $\mathcal{O}$-module is called the congruence module, and is denoted by $C_{0}\left(L ; V_{1}, V_{2}\right)$.

The following proposition follows from Deligne-Serre [7, Lemma 6.11] and will be used to construct congruences:

Proposition 4.10. - Keep the notations of Lemma 4.9. Let $\mathcal{T}$ be a commutative $\mathcal{O}$-algebra consisting of endomorphisms of $V$, preserving the lattice $L$ and the direct sum decomposition $V_{1} \oplus V_{2}$. For $j=1,2$, denote by $\mathcal{T}_{j}$ the image of $\mathcal{T}$ in $\operatorname{End}\left(V_{j}\right)$.

Assume that $C_{0}\left(L ; V_{1}, V_{2}\right)$ is non-zero and that its support contains $\mathcal{P}$.

Let $\mathfrak{m}_{1}$ be maximal ideal $\mathcal{T}_{1}$ of residue field $\kappa_{1}$, such that $L^{1} / L_{1} \otimes_{\mathcal{T}_{1}} \kappa_{1}$ is non zero, and denote by $\bar{\theta}_{1}: \mathcal{T}_{1} \rightarrow \kappa_{1}$ the corresponding character.

Then there exists a discrete valuation ring $\mathcal{O}^{\prime}$ of maximal ideal $\mathcal{P}^{\prime}$ (with $\mathcal{P}^{\prime} \cap \mathcal{O}=\mathcal{P}$ ), residue field $\kappa^{\prime} \supset \kappa_{1}$ and whose fraction field $E^{\prime}$ is a finite extension of $E$, and there exists a character $\theta_{2}: \mathcal{T}_{2} \rightarrow \mathcal{O}^{\prime}$ such that for each $T \in \mathcal{T}, \quad \bar{\theta}_{1}(T) \equiv \theta_{2}(T)\left(\bmod \mathcal{P}^{\prime}\right)$.

Proof. - For $j=1,2$, denote by $\pi_{j}$ the projection of $\mathcal{T}$ onto $\mathcal{T}_{j}$. Then $\mathfrak{m}=\pi_{1}^{-1}\left(\mathfrak{m}_{1}\right)$ is a maximal ideal of $\mathcal{T}$ of residue field $\kappa_{1}$. Put $\mathfrak{m}_{2}=\pi_{2}(\mathfrak{m})$. Since the isomorphism of Lemma 4.9(ii) is $\mathcal{T}$-equivariant, we have

$$
\left(L^{1} / L_{1}\right) \otimes_{\mathcal{T}_{1}}\left(\mathcal{T}_{1} / \mathfrak{m}_{1}\right) \cong\left(L /\left(L_{1} \oplus L_{2}\right)\right) \otimes_{\mathcal{T}}(\mathcal{T} / \mathfrak{m}) \cong\left(L^{2} / L_{2}\right) \otimes_{\mathcal{T}_{2}}\left(\mathcal{T}_{2} / \mathfrak{m}_{2}\right) .
$$

By assumption $\left(L^{1} / L_{1}\right) \otimes_{\mathcal{T}_{1}}\left(\mathcal{T}_{1} / \mathfrak{m}_{1}\right)$ is non-zero. Therefore $\mathfrak{m}_{2}$ is a maximal ideal of $\mathcal{T}_{2}$ of residue field $\kappa_{1}$ and the corresponding character $\bar{\theta}_{2}: \mathcal{T}_{2} \rightarrow \kappa_{1}$ fits in the following commutative diagram:

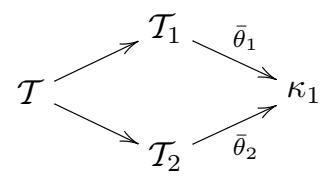

Since $\mathcal{T}_{2}$ is a (finite) flat $\mathcal{O}$-algebra, there exists a prime ideal $\mathcal{P}_{2}$, contained in $\mathfrak{m}_{2}$ and such that $\mathcal{P}_{2} \cap \mathcal{O}=0$. The reduction modulo $\mathcal{P}_{2}$ gives a character $\theta_{2}$ of $\mathcal{T}_{2}$ as in the statement.

THEOREM 4.11 (Theorem A). - Let $f$ and $p$ be such that (I), $\left(\mathbf{I r r}_{\bar{\rho}}\right)$ and (MW) hold, and $p-1>\max \left(1, \frac{5}{d}\right) \sum_{\tau \in J_{F}}\left(k_{\tau}-1\right)$. If $\iota_{p}\left(\frac{\Gamma\left(\operatorname{Ad}^{0}(f), 1\right) L^{*}\left(\operatorname{Ad}^{0}(f), 1\right)}{\Omega_{f}^{+} \Omega_{f}^{-}}\right) \in \mathcal{P}$, then $\mathcal{P}$ is a congruence prime for $f$.

Proof. - Let $L=\mathrm{H}_{!}^{d}\left(Y^{\text {an }}, \mathbb{V}_{n}(\mathcal{O})\right)_{\mathfrak{m}^{\prime}}^{\prime}\left[ \pm \hat{\epsilon}_{J_{0}}, \psi\right] \subset V=\mathrm{H}_{!}^{d}\left(Y^{\text {an }}, \mathbb{V}_{n}(E)\right)_{\mathfrak{m}^{\prime}}\left[ \pm \hat{\epsilon}_{J_{0}}, \psi\right]$ and $V_{1}=\mathrm{H}_{!}^{d}\left(Y^{\mathrm{an}}, \mathbb{V}_{n}(E)\right)\left[ \pm \hat{\epsilon}_{J_{0}}, f\right]$. Then $L_{1}=L \cap V_{1}=L_{f, J_{0}} \oplus L_{f, J_{F} \backslash J_{0}}$ (see Section 4.2). By (15) the twisted Poincaré pairing [, ] is non-degenerate on $V_{1}$. Let $V_{2}$ be the orthogonal subspace of $V_{1}$ in $V$.

$4^{\mathrm{e}}$ SÉRIE - TOME $38-2005-\mathrm{N}^{\circ} 4$ 
By Theorem 4.4(ii) the twisted Poincaré pairing is perfect on $\mathrm{H}_{!}^{d}\left(Y^{\mathrm{an}}, \mathbb{V}_{n}(\mathcal{O})\right)_{\mathfrak{m}^{\prime}}^{\prime}\left[ \pm \hat{\epsilon}_{J_{0}}\right]$. After rescaling by the factor $\frac{(f, f)_{\mathfrak{n}}}{(f, f)_{K_{1}(\mathfrak{n})}}$ (coming from congruences obtained by varying the central character) it restricts to a perfect pairing on $L$. Then, by [21, (4.6)] we have

$$
\left[L^{1}: L_{1}\right]=\left(\frac{(f, f)_{\mathfrak{n}}}{(f, f)_{K_{1}(\mathfrak{n})}}\right)^{2} \operatorname{disc}\left(L_{1}\right) .
$$

Using now (15), (19), and the assumption on $\mathcal{P}$ we obtain that the $\mathcal{O}$-module $L^{1} / L_{1}$ is nonzero and $\mathcal{P}$ belongs to its support. By Lemma 4.9 the same holds for the congruence module $C_{0}\left(L ; V_{1}, V_{2}\right)$. By Proposition 4.10 and the duality between $\mathbb{T}(\mathbb{C})$ and $S_{k}(\mathfrak{n}, \psi)$ there exists another normalized eigenform $g \in S_{k}(\mathfrak{n}, \psi)$ congruent to $f$. Hence $\mathcal{P}$ is a congruence prime for $f$.

\section{Fontaine-Laffaille weights of Hilbert modular varieties}

In this section all the objects are over $\mathcal{O}$. The aim is to establish a modulo $p$ version of Theorem 2.3 under the assumptions that $p$ does not divide $\Delta$ and $p-1>|n|+d$.

\subsection{The BGG complex over $\mathcal{O}$}

Koszul's complex. The Koszul's complex of the trivial $G$-module $\mathcal{O}$ is given by

$$
\cdots \rightarrow U_{\mathcal{O}}(\mathfrak{g}) \otimes \bigwedge_{\mathcal{O}}^{2} \mathfrak{g} \rightarrow U_{\mathcal{O}}(\mathfrak{g}) \otimes \mathfrak{g} \rightarrow U_{\mathcal{O}}(\mathfrak{g}) \rightarrow \mathcal{O} \rightarrow 0 .
$$

Since $\mathfrak{g}=\mathfrak{b} \oplus \mathfrak{u}^{-}$, the $\mathcal{O}[\mathfrak{b}]$-module $\mathfrak{g} / \mathfrak{b}$ is a direct factor in $\mathfrak{g}$ and we have a homomorphism of $B$-modules $U_{\mathcal{O}}(\mathfrak{g}) \otimes \bigwedge_{\mathcal{O}}^{\mathfrak{g}} \rightarrow U_{\mathcal{O}}(\mathfrak{g}) \otimes_{U_{\mathcal{O}}(\mathfrak{b})} \Lambda_{\mathcal{O}}(\mathfrak{g} / \mathfrak{b})$. Thus, we deduce another complex

$$
U_{\mathcal{O}}(\mathfrak{g}) \otimes_{U_{\mathcal{O}}(\mathfrak{b})} \bigwedge_{\mathcal{O}}(\mathfrak{g} / \mathfrak{b}) \rightarrow \mathcal{O} \rightarrow 0
$$

denoted by $S_{\mathcal{O}}^{\bullet}(\mathfrak{g}, \mathfrak{b})$.

More generally, for a free $\mathcal{O}$-module $V$ endowed with an action of $U_{\mathcal{O}}(\mathfrak{g})$, we consider the complex $S_{\mathcal{O}}(\mathfrak{g}, \mathfrak{b}) \otimes V$ endowed with the diagonal action of $U_{\mathcal{O}}(\mathfrak{g})$. For every $U_{\mathcal{O}}(\mathfrak{b})$-module $W$ which is free over $\mathcal{O}$, there is a canonical isomorphism of $U_{\mathcal{O}}(\mathfrak{g})$-modules

$$
\left(U_{\mathcal{O}}(\mathfrak{g}) \otimes_{U_{\mathcal{O}}(\mathfrak{b})} W\right) \otimes V \cong U_{\mathcal{O}}(\mathfrak{g}) \otimes_{U_{\mathcal{O}}(\mathfrak{b})}\left(\left.W \otimes V\right|_{\mathfrak{b}}\right) .
$$

Therefore we obtain another complex

$$
U_{\mathcal{O}}(\mathfrak{g}) \otimes_{U_{\mathcal{O}}(\mathfrak{b})}\left(\left.\bigwedge_{\mathcal{O}}(\mathfrak{g} / \mathfrak{b}) \otimes V\right|_{\mathfrak{b}}\right) \rightarrow V \rightarrow 0,
$$

denoted by $S_{\mathcal{O}}^{\bullet}(\mathfrak{g}, \mathfrak{b}, V)$. In the case where $V=V_{n}$ we denote it by $S_{\mathcal{O}}^{\bullet}(\mathfrak{g}, \mathfrak{b}, n)$.

Verma modules. For each weight $\mu \in \mathbb{Z}\left[J_{F}\right]$, we define a $U_{\mathcal{O}}(\mathfrak{g})$-module $V_{\mathcal{O}}(\mu):=$ $U_{\mathcal{O}}(\mathfrak{g}) \otimes_{U_{\mathcal{O}}(\mathfrak{b})} W_{\mu}(\mathcal{O})$, called the Verma module of weight $\mu$. 
LEMMA 5.1. - Let $W$ be a B-module, free of finite rank over $\mathcal{O}$, whose weights are smaller than $(p-1) t$. Then, there exists a filtration of B-modules $0=W_{0} \subset W_{1} \subset \cdots \subset W_{r}=W$ such that for every $1 \leqslant i \leqslant r$ there exists $\mu_{i} \in \mathbb{Z}\left[J_{F}\right]$ such that $W_{i} / W_{i+1} \cong W_{\mu_{i}}(\mathcal{O})$. Moreover the $W_{\mu_{i}}(\mathcal{O}), 1 \leqslant i \leqslant r$, are the irreducible factors of the $T$-module $W$.

In particular, if $U$ acts trivially on $W$, then $W \cong \bigoplus_{i=1}^{r} W_{\mu_{i}}(\mathcal{O})$.

Proof. - Let $\mu_{1}$ be a maximal weight of $W$ (for the partial order given by the positive roots of $G$ ) and let $v \in W$ be a $\mathcal{O}$-primitive vector of weight $\mu_{1}$. Let $W^{\prime}$ be the $U_{\mathcal{O}}(\mathfrak{b})$-submodule generated by $v$. Then $W^{\prime} \cong W_{\mu_{1}}(\mathcal{O})$ and $W^{\prime} \otimes \kappa$ is irreducible, because $\mu_{1}$ is smaller than $(p-1) t$ (and $W^{\prime}$ is free of rank 1). Since $W$ is free over $\mathcal{O}$ we have an exact sequence of $B$-modules

$$
0 \rightarrow \operatorname{Tor}_{1}^{\mathcal{O}}\left(W / W^{\prime}, \kappa\right) \rightarrow W^{\prime} \otimes \kappa \rightarrow W \otimes \kappa .
$$

Since $W^{\prime} \otimes \kappa$ is irreducible and $v$ is primitive, the last arrow is injective. Therefore

$$
\operatorname{Tor}_{1}^{\mathcal{O}}\left(W / W^{\prime}, \kappa\right)=0,
$$

that is $W / W^{\prime}$ is free over $\mathcal{O}$. The lemma follows then by induction.

LEMMA 5.2. - The module $S_{\mathcal{O}}^{i}(\mathfrak{g}, \mathfrak{b}, n)$ has a finite filtration by $U_{\mathcal{O}}(\mathfrak{g})$-submodules whose graded pieces are of the form $V_{\mathcal{O}}(\mu), \mu \in \Omega^{i}(n)$, where $\Omega^{i}(n)$ is the set of weights of the $\mathfrak{t}$-module $\left.\bigwedge_{\mathcal{O}}^{i}(\mathfrak{g} / \mathfrak{b}) \otimes V_{n}(\mathcal{O})\right|_{\mathfrak{b}}$.

Proof. - Since $p-1>|n|+d$ the previous lemma applies to $\left.\bigwedge_{\mathcal{O}}(\mathfrak{g} / \mathfrak{b}) \otimes V_{n}(\mathcal{O})\right|_{\mathfrak{b}}$. This gives a filtration $0=W_{0} \subset W_{1} \subset \cdots \subset W_{r}=\left.\bigwedge_{\mathcal{O}}^{i}(\mathfrak{g} / \mathfrak{b}) \otimes V_{n}(\mathcal{O})\right|_{\mathfrak{b}}$ whose graded pieces are $W_{\mu}(\mathcal{O})$, $\mu \in \Omega^{i}(n)$. Since $U_{\mathcal{O}}(\mathfrak{g})$ is $U_{\mathcal{O}}(\mathfrak{b})$-free, the functor $U_{\mathcal{O}}(\mathfrak{g}) \otimes_{U_{\mathcal{O}}(\mathfrak{b})} \bullet$ is exact.

Central characters. Let $U_{\mathcal{O}}(\mathfrak{g}) \rightarrow U_{\mathcal{O}}(\mathfrak{t})$ be the projection coming from the PoincaréBirkhoff-Witt decomposition $U_{\mathcal{O}}(\mathfrak{g})=U_{\mathcal{O}}(\mathfrak{t}) \oplus\left(\mathfrak{u}^{-} U_{\mathcal{O}}(\mathfrak{g})+U_{\mathcal{O}}(\mathfrak{g}) \mathfrak{u}\right)$. We take its restriction to the invariants for the adjoint action $\theta: U_{\mathcal{O}}(\mathfrak{g})^{G} \rightarrow U_{\mathcal{O}}(\mathfrak{t})$. Note that $U_{\overline{\mathbb{F}}_{p}}(\mathfrak{t})$ identifies with the algebra of regular functions on $\operatorname{Hom}_{\mathcal{O}}\left(\mathfrak{t}, \overline{\mathbb{F}}_{p}\right) \cong \overline{\mathbb{F}}_{p}\left[J_{F}\right]$ (a Laurent polynomial algebra). The Weyl group $\{ \pm 1\}^{J_{F}}$ of $G$ acts on it by $\left(\epsilon_{J} \cdot P\right)(\mu)=P\left(\epsilon_{J}(\mu+t)-t\right)$. The following result is analogous to the theorem of Harish-Chandra:

THEOREM 5.3 (Jantzen [28]). $-\theta_{\overline{\mathbb{F}}_{p}}$ induces an algebra isomorphism

$$
U_{\overline{\mathbb{F}}_{p}}(\mathfrak{g})^{G} \rightarrow U_{\overline{\mathbb{F}}_{p}}(\mathfrak{t})^{\{ \pm 1\}^{J_{F}}} .
$$

For every $\mu \in \mathbb{Z}\left[J_{F}\right]$ and every $\mathcal{O}$-algebra $R$, we denote by $d \mu_{R}: \mathfrak{t}_{R} \rightarrow R$ the corresponding character and by $\chi_{\mu, R}=d \mu_{R} \circ \theta_{R}$ the composed map $U_{R}(\mathfrak{g})^{G} \rightarrow U_{R}(\mathfrak{t}) \rightarrow R$. This definition is compatible with the $\mathcal{O}$-algebra homomorphisms.

If $V$ is a $U_{R}(\mathfrak{g})$-module generated by a vector $v$ of weight $\mu$ and annihilated by $\mathfrak{u}$, then $U_{R}(\mathfrak{g})^{G}$ acts over $V$ by $\chi_{\mu, R}$. Put $\chi_{\mu, p}=\chi_{\mu, \mathcal{O}}$ and $\bar{\chi}_{\mu, p}=\chi_{\mu, \overline{\mathbb{F}}_{p}}$.

COROLlary 5.4. - If $\bar{\chi}_{n, p}=\bar{\chi}_{\mu, p}$, then there exists $J \subset J_{F}$ such that $\mu-\left(\epsilon_{J}(n+t)-t\right) \in$ $p \mathbb{Z}\left[J_{F}\right]$. In particular, if $\mu$ is smaller than $(p-1) t$, then we have $\mu=\epsilon_{J}(n+t)-t$.

Proposition 5.5. - Let $\mu \in \Omega^{i}(n)$ (see Lemma 5.2). Then $\bar{\chi}_{n, p}=\bar{\chi}_{\mu, p}$ if, and only if, there exists a subset $J \subset J_{F}$ containing $i$ elements and such that $\mu=\epsilon_{J}(n+t)-t$.

Proof. - By the corollary, it remains to show that for $J \subset J_{F}$, we have $\epsilon_{J}(n+t)-t \in$ $\Omega^{i}(n)$ if, and only if, $|J|=i$. By Lemma 5.2, we have to show that $W_{\epsilon_{J}(n+t)-t, n_{0}}(E)$

$4^{\mathrm{e}}$ SÉRIE - TOME $38-2005-\mathrm{N}^{\circ} 4$ 
occurs in $\left.\bigwedge_{E}^{i}(\mathfrak{g} / \mathfrak{b}) \otimes V_{n}(E)\right|_{\mathfrak{t}}$ (with multiplicity one) if and only if $|J|=i$. The weights of $\left.\bigwedge_{E}^{i}(\mathfrak{g} / \mathfrak{b}) \otimes V_{n}(E)\right|_{\mathfrak{t}}$ are of the form $\epsilon_{J^{\prime}}(n+t)-t+\nu$, where $J^{\prime} \subset J_{F}$ is a subset containing $i$ elements and $\nu$ is a weight of $V_{n}(E)$. Therefore $\epsilon_{J}(n+t)-t=\epsilon_{J^{\prime}}(n+t)-t+\nu$ and so $n=\epsilon_{J}(\nu)+\epsilon_{J}\left(\epsilon_{J^{\prime}}(t)\right)-t$. Since $n$ is a maximal weight of $V_{n}(E)$, we deduce that $J=J^{\prime}$.

Decomposition with respect to central characters. By Lemma 5.2, $S_{\mathcal{O}}^{i}(\mathfrak{g}, \mathfrak{b}, n)$ admits a finite filtration by $U_{\mathcal{O}}(\mathfrak{g})$-submodules with graded of the form $V_{\mathcal{O}}(\mu), \mu \in \Omega^{i}(n)$. Therefore $S_{\mathcal{O}}(\mathfrak{g}, \mathfrak{b}, n)$ is annihilated by a power of the ideal $I:=\prod_{\mu \in \Omega} \bullet{ }_{(n)} \operatorname{ker}\left(\chi_{\mu, p}\right)$ of the commutative ring $U_{\mathcal{O}}(\mathfrak{g})^{G}$. In fact, it would follow from Proposition 5.5 that $S_{\mathcal{O}}^{\bullet}(\mathfrak{g}, \mathfrak{b}, n)$ is annihilated by $I$ itself. We have the following commutative algebra result:

Lemma 5.6. - Let $P_{1}, \ldots, P_{r}$ be ideals of a commutative ring $R$ such that $P_{1} \ldots P_{r}=0$ and for all $i \neq j, P_{i}+P_{j}=R$. Then each $R$-module $W$ admits a direct sum decomposition $W=\bigoplus_{1 \leqslant i \leqslant r} W^{P_{i}}$, with $W^{P_{i}}=\left\{m \in W \mid P_{i} m=0\right\}$.

Consider the maximal ideals $\left(p, \operatorname{ker}\left(\chi_{\mu, p}\right)\right)=\operatorname{ker}\left(\bar{\chi}_{\mu, p}\right)$ of $U_{\mathcal{O}}(\mathfrak{g})^{G}$, where $\mu \in \Omega^{\bullet}(n)$. Let $\bar{\chi}_{1}=\bar{\chi}_{n, p}, \bar{\chi}_{2}, \ldots, \bar{\chi}_{r}$ be the set of distinct characters among $\bar{\chi}_{\mu, p}, \mu \in \Omega^{\bullet}(n)$. Put $P_{i}=$ $\prod_{\bar{\chi}_{\mu, p}=\bar{\chi}_{i}} \operatorname{ker}\left(\chi_{\mu, p}\right)$. By the above lemma we get a decomposition

$$
S_{\mathcal{O}}^{\bullet}(\mathfrak{g}, \mathfrak{b}, n)=\bigoplus_{i=1}^{r} S_{\mathcal{O}}^{\bullet}(\mathfrak{g}, \mathfrak{b}, n)^{P_{i}}
$$

which is a direct sum, because the differentials are $U_{\mathcal{O}}(\mathfrak{g})$-equivariant. Moreover, $V_{\mathcal{O}}(\mu)_{\bar{\chi}_{n, p}}=$ $V_{\mathcal{O}}(\mu)$ if $\bar{\chi}_{\mu, p}=\bar{\chi}_{n, p}$, and $V_{\mathcal{O}}(\mu)_{\bar{\chi}_{n, p}}=0$ otherwise. From here and from Proposition 5.5 we get:

THEOREM 5.7. - The complex $S_{\mathcal{O}}^{\bullet}(\mathfrak{g}, \mathfrak{b}, n)_{\bar{\chi}_{n, p}}$ is a direct factor in $S_{\mathcal{O}}^{\bullet}(\mathfrak{g}, \mathfrak{b}, n)$ and we have $S_{\mathcal{O}}^{0}(\mathfrak{g}, \mathfrak{b}, n)_{\bar{\chi}_{n, p}}=V_{n}(\mathcal{O})$. For each $i \geqslant 1, S_{\mathcal{O}}^{i}(\mathfrak{g}, \mathfrak{b}, n)_{\bar{\chi}_{n, p}}$ has a filtration whose graded are given by the $V_{\mathcal{O}}\left(\epsilon_{J}(n+t)-t\right.$ ) where $J \subset J_{F},|J|=i$ (with multiplicity one).

\subsection{The BGG complex for distributions algebras}

Let $\mathcal{U}_{\mathcal{O}}(G)$ be the distribution $\mathcal{O}$-algebra over $G$. For each $G$-module $V$, free over $\mathcal{O}$, we define the complex

$$
0 \leftarrow V \leftarrow \mathcal{U}_{\mathcal{O}}(G) \otimes \mathcal{U}_{\mathcal{O}}(B)\left(\grave{\mathcal{O}}_{\left.\left.(\mathfrak{g} / \mathfrak{b}) \otimes V\right|_{\mathfrak{b}}\right),}\right.
$$

and denote it by $\mathcal{S}_{\mathcal{O}}^{\bullet}(G, B, V)$. In the case where $V=V_{n}(\mathcal{O})$ we denote this complex by $\mathcal{S}_{\mathcal{O}}^{\bullet}(G, B, n)$.

Remark 5.8. - The complex $\mathcal{S}_{\mathcal{O}}^{\bullet}(G, B, V)$ is not exact. It will become exact after applying the Grothendieck linearization functor to the associated complex of vector bundles over the Hilbert modular variety.

For all $\mu \in \mathbb{Z}\left[J_{F}\right]$, we define the Verma module $\mathcal{V}_{\mathcal{O}}(\mu)=\mathcal{U}_{\mathcal{O}}(G) \otimes_{\mathcal{U}_{\mathcal{O}}(B)} W_{\mu}(\mathcal{O})$ (see Section 5.1). We recall that, since $p-1>|n|+d, \Omega^{i}(n)$ is the set of $\mu \in \mathbb{Z}\left[J_{F}\right]$ such that $W_{\mu}(\mathcal{O})$ is an irreducible subquotient of $\left.\bigwedge_{\mathcal{O}}^{i}(\mathfrak{g} / \mathfrak{b}) \otimes V_{n}(\mathcal{O})\right|_{\mathfrak{b}}$. Lemma 5.2 translates as:

LEMma 5.9. - The module $\mathcal{S}_{\mathcal{O}}^{\bullet}(G, B, n)$ has a finite filtration by $\mathcal{U}_{\mathcal{O}}(G)$-submodules whose successive quotients are given by $\mathcal{V}_{\mathcal{O}}(\mu)$, with $\mu \in \Omega^{i}(n)$. 
Since $U_{\mathcal{O}}(\mathfrak{g}) \subset \mathcal{U}_{\mathcal{O}}(G) \subset U_{E}(\mathfrak{g})$, the center $U_{\mathcal{O}}(\mathfrak{g})^{G}$ of $U_{\mathcal{O}}(\mathfrak{g})$ is contained in the center of $\mathcal{U}_{\mathcal{O}}(G)$. Consider the central characters $\chi_{\mu, p}=\chi_{\mu, \mathcal{O}}$ and $\bar{\chi}_{\mu, p}=\chi_{\mu, \overline{\mathbb{F}}_{p}}$ (see Section 5.1).

If $W$ is a $\mathcal{U}_{\mathcal{O}}(G)$-module generated by a vector $v$ of weight $\mu$ and annihilated by $\mathfrak{u}$, then $U_{\mathcal{O}}(\mathfrak{g})^{G}$ acts on $W$ by the character $\chi_{\mu, p}$. Put $I=\prod_{\mu \in \Omega \bullet(n)} \operatorname{ker}\left(\chi_{\mu, p}\right)$. By the last lemma the finite $\mathcal{O}$-module $\mathcal{S}_{\mathcal{O}}^{\bullet}(G, B, n)$ is a $R:=U_{\mathcal{O}}(\mathfrak{g})^{G} / I$-module. Let $\bar{\chi}_{1}=\bar{\chi}_{n, p}, \bar{\chi}_{2}, \ldots, \bar{\chi}_{r}$ be the distinct algebra homomorphisms from $R$ in $\overline{\mathbb{F}}_{p}$. For $1 \leqslant j \leqslant r$, we put

$$
\mathcal{S}_{\mathcal{O}}^{\bullet}(G, B, n)_{\bar{\chi}_{j}}=\left\{x \in \mathcal{S}_{\mathcal{O}}^{\bullet}(G, B, n) \mid\left(\prod_{\mu \in \Omega \cdot(n), \bar{\chi}_{\mu, p}=\bar{\chi}_{j}} \operatorname{ker}\left(\chi_{\mu, p}\right)\right) x=0\right\} .
$$

The same way as in Theorem 5.7 we obtain a decomposition:

$$
\mathcal{S}_{\mathcal{O}}^{\bullet}(G, B, n)=\bigoplus_{j=1}^{r} \mathcal{S}_{\mathcal{O}}^{\bullet}(G, B, n)_{\bar{\chi}_{j}}
$$

THEOREM 5.10. $-\mathcal{S}_{\mathcal{O}}^{i}(G, B, n)_{\bar{\chi}_{n, p}} \cong \bigoplus_{J \subset J_{F},|J|=i} \mathcal{V}_{\mathcal{O}}\left(\epsilon_{J}(n+t)-t\right)$.

Proof. - Assume first $n=0$. Since $\mathfrak{u}$ is Abelian, $U$ acts trivially on $\bigwedge_{\mathcal{O}}^{i}(\mathfrak{g} / \mathfrak{b})$ and Lemma 5.2 gives $\bigwedge_{\mathcal{O}}^{i}(\mathfrak{g} / \mathfrak{b}) \cong \bigoplus_{J \subset J_{F},|J|=i} W_{\epsilon_{J}(t)-t}(\mathcal{O})$. Since $\mathcal{U}_{\mathcal{O}}(G)$ is free over $\mathcal{U}_{\mathcal{O}}(B)$ we obtain:

$$
\mathcal{S}_{\mathcal{O}}^{i}(G, B, 0)=\mathcal{S}_{\mathcal{O}}^{i}(G, B, 0)_{\bar{\chi}_{0, p}} \cong \bigoplus_{J \subset J_{F},|J|=i} \mathcal{V}_{\mathcal{O}}\left(\epsilon_{J}(t)-t\right)
$$

For $n \geqslant 0$, using the $n=0$ case, we already have a decomposition:

$$
\mathcal{S}_{\mathcal{O}}^{i}(G, B, n) \cong \bigoplus_{J \subset J_{F},|J|=i} \mathcal{U}_{\mathcal{O}}(G) \otimes_{\mathcal{U}_{\mathcal{O}}(B)}\left(W_{\epsilon_{J}(t)-t}(\mathcal{O}) \otimes V_{n}(\mathcal{O})\right)
$$

By (20), the theorem is a consequence of the following lemma, whose proof follows directly from the one of Proposition 5.5.

LEMMA 5.11. - $\left(\mathcal{U}_{\mathcal{O}}(G) \otimes_{\mathcal{U}_{\mathcal{O}}(B)}\left(W_{\epsilon_{J}(t)-t}(\mathcal{O}) \otimes V_{n}(\mathcal{O})\right)\right)_{\bar{\chi}_{n, p}} \cong \mathcal{V}_{\mathcal{O}}\left(\epsilon_{J}(n+t)-t\right)$.

\subsection{BGG complex for crystals}

Our reference is [31, Section 4]. For every integer $r \geqslant 0$ we put $S_{r}=\operatorname{Spec}\left(\mathbb{Z} / p^{r+1}\right)$. For a $\mathbb{Z}\left[\frac{1}{\Delta}\right]$-scheme $X$, we put $X_{r}=X \times S_{r}$.

We have an equivalence of categories between the category of crystals over $\left(\bar{X}_{0} / S_{r}\right)_{\log }^{\text {crys }}$ and the category of $\mathcal{O}_{\bar{X}_{r}}$-modules $\mathcal{M}$ which are locally free and endowed with integrable, quasiunipotent connection with logarithmic poles $\nabla: \mathcal{M} \rightarrow \mathcal{M} \otimes_{\mathcal{O}_{\bar{X}_{r}}} \Omega_{\bar{X}_{r} / S_{r}}\left(\operatorname{dlog}\left(\infty_{X}\right)\right)$.

We have a functor L, called the linearization functor, from the category of sheaves of $\mathcal{O}_{\bar{X}_{r}}$-modules to the category of crystals on $\left(\bar{X}_{0} / S_{r}\right)_{\log }^{\text {crys }}$.

By the log-crystalline Poincare lemma, we have a resolution:

$$
0 \rightarrow \mathcal{M} \rightarrow \mathrm{L}\left(\mathcal{M} \otimes \mathcal{O}_{\bar{X}_{r}} \Omega_{\bar{X}_{r} / S_{r}}(\operatorname{dlog} \infty)\right)
$$

$4^{\mathrm{e}}$ SÉRIE - TOME $38-2005-\mathrm{N}^{\circ} 4$ 
Let $W_{1}$ and $W_{2}$ be two $B$-modules with weights smaller than $(p-1) t$. Put $\overline{\mathcal{W}}_{i}=\mathcal{F}_{B}\left(W_{i}\right)$, $i=1,2$ (see Section 2.3). By [31, §5.2.4] we have a homomorphism

$$
\operatorname{Hom}_{\mathcal{U}_{\mathcal{O}}(G)}\left(\mathcal{U}_{\mathcal{O}}(G) \otimes_{\mathcal{U}_{\mathcal{O}}(B)} W_{1}\right),\left(\mathcal{U}_{\mathcal{O}}(G) \otimes_{\mathcal{U}_{\mathcal{O}}(B)} W_{2}\right) \rightarrow \operatorname{Diff.Op} .\left(\overline{\mathcal{W}}_{2, r}, \overline{\mathcal{W}}_{1, r}\right)
$$

which becomes an isomorphism after tensoring with $E$ (see (12)).

We apply now the above construction to the toroidal compactification of the Hilbert modular variety $\bar{M}^{\prime}$ and the vector bundle $\overline{\mathcal{V}}_{n}$. For every $r \geqslant 0$ we have an injective homomorphism of complexes of vector bundles over $\bar{M}_{r}^{\prime}$

$$
\mathcal{K}_{n}^{\bullet}:=\bigoplus_{J \subset J_{F}} \overline{\mathcal{W}}_{\epsilon_{J}(n+t)-t, n_{0}} \hookrightarrow \overline{\mathcal{V}}_{n} \otimes_{\mathcal{O}_{\bar{M}_{r}^{\prime}}} \Omega_{\bar{M}_{r}^{\prime} / S_{r}}(\operatorname{dlog} \infty)
$$

PROPOSITION 5.12. - The map (21) is a strict injective homomorphism of filtered complexes.

By the last proposition $\mathrm{L}\left(\mathcal{K}_{n}^{\bullet}\right)$ is a direct factor in $\mathrm{L}\left(\overline{\mathcal{V}}_{n} \otimes_{\mathcal{O}_{\bar{M}_{r}^{\prime}}} \Omega_{\bar{M}_{r}^{\prime} / S_{r}}^{\bullet}(\operatorname{dlog} \infty)\right)$, which is exact by the Poincaré's crystalline lemma. Therefore $\mathrm{L}\left(\mathcal{K}_{n}^{\bullet}\right)$ is also exact. Since the functor $\mathrm{L}$ is exact, we deduce filtered isomorphisms $\mathcal{H}_{\log -\mathrm{dR}}^{j}\left(\bar{M}_{r}^{\prime} / S_{r}, \overline{\mathcal{V}}_{n}\right) \cong \mathcal{H}^{j}\left(\bar{M}_{r}^{\prime} / S_{r}, \mathcal{K}_{n}^{\bullet}\right)$.

Recall that $p$ does not divide $\Delta$ and $p-1>|n|+d$. Under this assumption we have

THEOREM 5.13. - The spectral sequence given by the Hodge filtration

$$
\mathrm{E}_{1}^{i, j}=\bigoplus_{J \subset J_{F},|p(J)|=i} \mathrm{H}^{i+j-|J|}\left(\bar{M}_{r}^{\prime}, \overline{\mathcal{W}}_{\epsilon_{J}(n+t)-t, n_{0}}\right) \Longrightarrow \mathcal{H}_{\log -\mathrm{dR}}^{i+j}\left(\bar{M}_{r}^{\prime}, \overline{\mathcal{V}}_{n}\right)
$$

degenerates at $\mathrm{E}_{1}$ for $r=0$ :

$$
\operatorname{gr}^{i} \mathcal{H}_{\log -\mathrm{dR}}^{j}\left(\bar{M}_{/ \overline{\mathbb{F}}_{p}}, \overline{\mathcal{V}}_{n}\right)=\bigoplus_{J \subset J_{F},|J| \leqslant j,|p(J)|=i} \mathrm{H}^{j-|J|}\left(\bar{M}_{/ \overline{\mathbb{F}}_{p}}, \overline{\mathcal{W}}_{\epsilon_{J}(n+t)-t, n_{0}}\right)
$$

Proof. - The proof is formally the same as the one of Theorem 2.3(ii), once we have Proposition 5.12. The degeneration of the spectral sequence follows from a result of Illusie [27, Proposition 4.13.] applied to the semi-stable morphism $\bar{\pi}_{s}: \overline{\mathcal{A}^{s}} \rightarrow \overline{M^{1}}$ of smooth $\mathbb{Z}_{p}$-schemes.

Remark 5.14.-

(i) It follows from the same arguments as in Corollary 2.7(i), that the above decomposition is Hecke equivariant, except for the $T_{\mathfrak{p}}$ operators, when $\mathfrak{p}$ divides $p$. When $p$ is totally split in $F$, we could use Wedhorn's results [42] to write $T_{\mathfrak{p}}$ as a sum of correspondences and try to adapt to this case the method of [16]. Unfortunately, this approach is not available when $p$ is not totally split in $F$.

In the proof of Theorem 6.7, we will use a different method to prove the $T_{\mathfrak{p}}$-equivariance of the above decomposition after a localization outside $p$.

(ii) The commutativity of the Hecke operators outside $p$ follows from the degeneration at $E_{1}$ as in the proof of Corollary 2.7(i). The last graded piece $\mathrm{H}^{0}\left(\bar{Y}, \overline{\mathcal{W}}_{\epsilon_{J_{F}}}(n+t)-t, n_{0}\right)$ of the filtration is independent of the toroidal compactification by the Koecher Principle (3). 


\section{Integral cohomology over certain local components of the Hecke algebra}

\subsection{The key lemma}

Let $q=p^{r}$ and denote by $\sigma_{1}, \ldots, \sigma_{r}$ the elements of $\operatorname{Gal}\left(\mathbb{F}_{q} / \mathbb{F}_{p}\right)$.

TheOREM 6.1 (Brauer-Nesbitt, Steinberg [39]). - The group $\mathrm{SL}_{2}\left(\mathbb{F}_{q}\right)$ has exactly q irreducible representations on finite-dimensional $\mathbb{F}_{q}$-vector spaces, namely the $\bigotimes_{j=1}^{r}\left(\operatorname{Sym}^{a_{j}}\right)^{\sigma_{j}}$, for $0 \leqslant a_{j} \leqslant p-1$.

COROLlaRY 6.2. - For every finite set $I$, the group $\prod_{i \in I} \mathrm{SL}_{2}\left(\mathbb{F}_{q}\right)$ has exactly $q^{|I|}$ irreducible representations on finite-dimensional $\mathbb{F}_{q}$-vector spaces, namely the

$$
\bigotimes_{i \in I}\left(\bigotimes_{j=1}^{r}\left(\operatorname{Sym}_{i}^{a_{i, j}}\right)^{\sigma_{j}}\right), \quad \text { for } 0 \leqslant a_{i, j} \leqslant p-1
$$

In [30] Mazur states the following:

LEMMA 6.3. - Let $\Phi$ be a group and let $\rho_{0}$ be a representation of $\Phi$ on a finite-dimensional $\mathbb{F}_{q}$-vector space $W$. Let $\rho: \Phi \rightarrow \mathrm{GL}_{2}\left(\mathbb{F}_{q}\right)$ be an absolutely irreducible representation such that for all $y \in \Phi$, the characteristic polynomial of $\rho(y)$ annihilates $\rho_{0}(y)$. Then, $\rho_{0}^{\text {s.s. }}=\rho \oplus \cdots \oplus \rho$ and in particular $\rho \subset \rho_{0}$.

The corresponding statement for another group than $\mathrm{GL}_{2}$ is false in general. Here is an example for $\mathrm{GL}_{3}$ : take $\rho=\mathrm{Sym}^{2}: \mathrm{GL}_{2}\left(\mathbb{F}_{q}\right) \rightarrow \mathrm{GL}_{3}\left(\mathbb{F}_{q}\right)$ and $\rho_{0}=\operatorname{det}: \mathrm{GL}_{2}\left(\mathbb{F}_{q}\right) \rightarrow \mathrm{GL}_{1}\left(\mathbb{F}_{q}\right)$. Nevertheless, we have a generalization for the special group:

$$
H\left(\mathbb{F}_{q}\right)=\left(\prod_{i \in I} \mathrm{GL}_{2}\left(\mathbb{F}_{q}\right)\right)^{\mathcal{D}}:=\left\{\left(M_{i}\right)_{i \in I} \in \prod_{i \in I} \mathrm{GL}_{2}\left(\mathbb{F}_{q}\right) \mid \exists \delta \in \mathcal{D}, \forall i \in I, \operatorname{det}\left(M_{i}\right)=\delta\right\}
$$

and the particular representation

$$
\rho_{1}=\bigotimes_{i \in I, \tau \in J_{F}^{i}} \mathrm{St}_{i}^{\sigma_{i, \tau}}: H\left(\mathbb{F}_{q}\right) \rightarrow \mathrm{GL}_{2^{d}}\left(\mathbb{F}_{q}\right), \quad\left(M_{i}\right)_{i \in I} \mapsto \bigotimes_{i \in I, \tau \in J_{F}^{i}} M_{i}^{\sigma_{i, \tau}},
$$

where $\left(J_{F}^{i}\right)_{i \in I}$ is a partition of $J_{F}$ and for all $i \in I,\left(\sigma_{i, \tau}\right)_{\tau \in J_{F}^{i}}$ are two by two distinct elements of $\operatorname{Gal}\left(\mathbb{F}_{q} / \mathbb{F}_{p}\right)\left(\mathrm{St}=\mathrm{Sym}^{1}\right.$ denotes the standard representation of $\left.\mathrm{GL}_{2}\right)$.

LEMMA 6.4. - Let $\rho_{0}$ be a representation of $H\left(\mathbb{F}_{q}\right)$ on a finite-dimensional $\mathbb{F}_{q}$-vector space $W$, such that for all $y \in H\left(\mathbb{F}_{q}\right)$ the characteristic polynomial of $\rho_{1}(y)$ annihilates $\rho_{0}(y)$. Then $\rho_{0}^{\text {s.s. }}=\rho_{1} \oplus \cdots \oplus \rho_{1}$ (each irreducible subquotient of $\rho_{0}$ is isomorphic to $\rho_{1}$ ).

Proof. - We can assume that $\rho_{0}$ is absolutely irreducible. Consider the exact sequence $1 \rightarrow H_{1}\left(\mathbb{F}_{q}\right)=\prod_{i \in I} \mathrm{SL}_{2}\left(\mathbb{F}_{q}\right) \rightarrow H\left(\mathbb{F}_{q}\right) \stackrel{\nu}{\rightarrow} \mathcal{D} \rightarrow 1$. By Corollary 6.2, we know that each irreducible subquotient of $\rho_{0 \mid H_{1}\left(\mathbb{F}_{q}\right)}$ is of the form $\bigotimes_{i \in I}\left(\bigotimes_{j=1}^{r}\left(\operatorname{Sym}_{i}^{a_{i, j}}\right)^{\sigma_{j}}\right)$, with $0 \leqslant a_{i, j} \leqslant$ $p-1$.

The subspace corresponding to the highest weight of the representation $\rho_{0 \mid H_{1}\left(\mathbb{F}_{q}\right)}$ is preserved by the standard torus of $H\left(\mathbb{F}_{q}\right)$ and therefore contains an eigenvector $x$ for the action of this torus. Since $\rho_{0}$ is irreducible, it is generated by $x$, and therefore $\rho_{0}$ is isomorphic to a twist of $\bigotimes_{i \in I}\left(\bigotimes_{j=1}^{r}\left(\operatorname{Sym}_{i}^{a_{i, j}}\right)^{\sigma_{j}}\right)$ by some power of the character $\nu$ (in particular $\rho_{0 \mid H_{1}\left(\mathbb{F}_{q}\right)}$ is also irreducible).

$4^{\text {e }}$ SÉRIE - TOME $38-2005-\mathrm{N}^{\circ} 4$ 
Since the characteristic polynomial of $\rho_{1}$ annihilates $\rho_{0}$, the set of the weights of $\rho_{0}$ is a subset of the set of the weights of $\rho_{1}$, and therefore $\rho_{0}=\rho_{1}$.

In Section 3.5 we proved under the assumption $\left(\mathbf{L I}_{\operatorname{Ind} \bar{\rho}}\right)$ that $\operatorname{Ind}_{F}^{\mathbb{Q}} \bar{\rho}\left(\mathcal{G}_{\widehat{F}}\right)$ contains the image of the map $\phi=\left(\phi^{i}\right)_{i \in I}: H\left(\mathbb{F}_{q}\right) \hookrightarrow \mathrm{GL}_{2}\left(\mathbb{F}_{q}\right)^{J_{F}}$.

Denote by $\widehat{F}^{\prime}$ the fixed field of $\bar{\rho}^{-1}\left(\phi\left(H\left(\mathbb{F}_{q}\right)\right)\right)$.

LEMMA 6.5 (Key lemma). - Let $\rho_{0}$ be a representation of $\mathcal{G}_{\widehat{F}}$, on a finite-dimensional $\kappa$-vector space $W$. Assume $\left(\mathbf{L I}_{\text {Ind } \bar{\rho}}\right)$ and assume that, for every $y \in \mathcal{G}_{\widehat{F}^{\prime}}$, the characteristic polynomial of $\left(\otimes \operatorname{Ind}_{F}^{\mathbb{Q}} \bar{\rho}\right)(y)$ annihilates $\rho_{0}(y)$. Then each $\mathcal{G}_{\widehat{F}^{\prime}}$-irreducible subquotient of $\rho_{0}$ is isomorphic to $\otimes \operatorname{Ind}_{F}^{\mathbb{Q}} \bar{\rho}$.

Proof. - It is enough to treat the case where $\rho_{0}$ is irreducible. The idea is show that the action of $\mathcal{G}_{\widehat{F}^{\prime}}$ on $W$ is through the algebraic group $H\left(\mathbb{F}_{q}\right)$ and use Lemma 6.4.

Put $\bar{\rho}^{\prime}=\left(\operatorname{Ind}_{F}^{\mathbb{Q}} \bar{\rho}\right)_{\mid \mathcal{G}_{F^{\prime}}}$. By the annihilation assumption, the group $\rho_{0}\left(\operatorname{ker}\left(\bar{\rho}^{\prime}\right)\right)$ is an unipotent $p$-group and therefore $W^{\operatorname{ker}\left(\bar{\rho}^{\prime}\right)}$ is non-zero. Moreover the subspace $W^{\operatorname{ker}\left(\bar{\rho}^{\prime}\right)}$ is preserved by $\mathcal{G}_{\widehat{F}^{\prime}}$. Since $W$ is irreducible we get $W^{\operatorname{ker}\left(\bar{\rho}^{\prime}\right)}=W$ and therefore the action of $\mathcal{G}_{\widehat{F}^{\prime}}$ on $W$ is through $H\left(\mathbb{F}_{q}\right)$. Hence there exists a homomorphism $\rho_{0}^{\prime}$ fitting in the following commutative diagram:

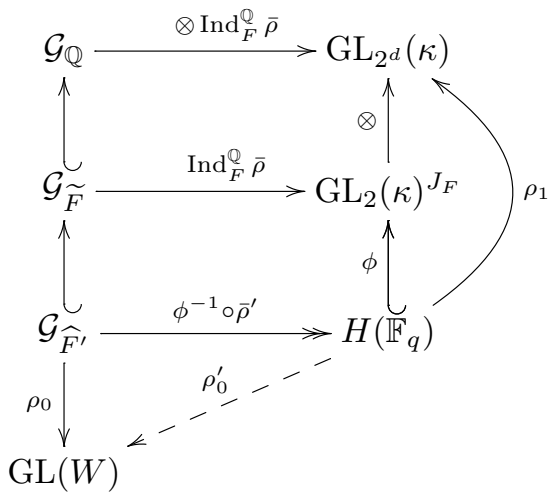

The characteristic polynomial of $\rho_{1}$ annihilates the representation $\rho_{0}^{\prime}$. By Lemma 6.4 each $H$ irreducible subquotient of $W$ is isomorphic to $\rho_{1}$, that is to say $W^{\text {s.s. }}=\bigoplus \rho_{1}$ as $H\left(\mathbb{F}_{q}\right)$-modules. Since the action of $\mathcal{G}_{\widehat{F}^{\prime}}$ on both sides is through $H\left(\mathbb{F}_{q}\right)$, we are done.

\subsection{Localized cohomology of the Hilbert modular variety}

Let $\mathbb{T}^{\prime} \subset \mathbb{T}$ be the subalgebra generated by the Hecke operators outside a finite set of places containing those dividing $\mathfrak{n} p$. Put $\mathfrak{m}^{\prime}=\mathfrak{m} \cap \mathbb{T}^{\prime}$.

THEOREM 6.6. - Assume $f$ and $p$ satisfy $(\mathbf{I})$, (II) and $\left(\mathbf{L I}_{\text {Ind } \bar{\rho}}\right)$. Then

(i) $\mathrm{H}^{\bullet}\left(Y, \mathbb{V}_{n}(\kappa)\right)_{\mathfrak{m}^{\prime}}=\mathrm{H}^{d}\left(Y, \mathbb{V}_{n}(\kappa)\right)_{\mathfrak{m}^{\prime}}$,

(ii) $\mathrm{H}^{\bullet}\left(Y, \mathbb{V}_{n}(\mathcal{O})\right)_{\mathfrak{m}^{\prime}}=\mathrm{H}^{d}\left(Y, \mathbb{V}_{n}(\mathcal{O})\right)_{\mathfrak{m}^{\prime}}$ is a free $\mathcal{O}$-module of finite rank and the $\mathcal{O}$-module $\mathrm{H}^{\bullet}\left(Y, \mathbb{V}_{n}(E / \mathcal{O})\right)_{\mathfrak{m}^{\prime}}=\mathrm{H}^{d}\left(Y, \mathbb{V}_{n}(E / \mathcal{O})\right)_{\mathfrak{m}^{\prime}}$ is divisible of finite corank.

(iii) $\mathrm{H}^{d}\left(Y, \mathbb{V}_{n}(\mathcal{O})\right)_{\mathfrak{m}^{\prime}} \times \mathrm{H}^{d}\left(Y, \mathbb{V}_{n}(E / \mathcal{O})\right)_{\mathfrak{m}^{\prime}} \rightarrow \mathcal{O}$ is a perfect Pontryagin pairing.

Proof. - (i) By Faltings' Comparison Theorem [14] and Theorem 5.13(i) the integer $|p(J)|$ is not a Fontaine-Laffaille weight of $\mathrm{H}^{r}(\kappa)$ when $r<d$. Wedhorn [42] has established the congruence relations for all totally split primes of $F$. By the Cebotarev Density Theorem the 
assumptions of the key Lemma 6.5 are fulfilled. We deduce that $\mathrm{H}^{r}(\kappa)\left[\mathfrak{m}^{\prime}\right]=0$ and therefore by Nakayama's lemma $\mathrm{H}^{r}(\kappa)_{\mathfrak{m}^{\prime}}=0$. The case $n>d$ follows by Poincaré duality.

(ii), (iii) By the long exact cohomology sequence

$$
\cdots \rightarrow \mathrm{H}^{r-1}(\kappa) \rightarrow \mathrm{H}^{r}(\mathcal{O}) \stackrel{\varpi}{\longrightarrow} \mathrm{H}^{r}(\mathcal{O}) \rightarrow \mathrm{H}^{r}(\kappa) \rightarrow \cdots,
$$

and by the vanishing of $\mathrm{H}^{r}(\kappa)_{\mathfrak{m}^{\prime}}$ for $r \neq d$, we deduce that (for $r \neq d$ ) the multiplication by an uniformizer $\varpi$ is a surjective endomorphism of $\mathrm{H}^{r}(\mathcal{O})_{\mathfrak{m}^{\prime}}$, so this last vanishes.

The same way, by the long exact sequence

$$
\cdots \rightarrow \mathrm{H}^{r}\left(\varpi^{-1} \mathcal{O} / \mathcal{O}\right) \rightarrow \mathrm{H}^{r}(E / \mathcal{O}) \stackrel{\varpi}{\longrightarrow} \mathrm{H}^{r}(E / \mathcal{O}) \rightarrow \mathrm{H}^{r+1}\left(\varpi^{-1} \mathcal{O} / \mathcal{O}\right) \rightarrow \cdots,
$$

we deduce a surjection $\mathrm{H}^{r}(\kappa)_{\mathfrak{m}^{\prime}} \rightarrow \mathrm{H}^{r}(E / \mathcal{O})_{\mathfrak{m}^{\prime}}[\varpi]$ for $r \neq d$. Since $\mathrm{H}^{r}(E / \mathcal{O})_{\mathfrak{m}^{\prime}}$ is a torsion $\mathcal{O}$-module, it vanishes (for $r \neq d$ ).

The localization at $\mathfrak{m}^{\prime}$ of the long exact sequence of $\mathcal{O}$-modules:

$$
\cdots \rightarrow \mathrm{H}^{r-1}(E / \mathcal{O}) \rightarrow \mathrm{H}^{r}(\mathcal{O}) \rightarrow \mathrm{H}^{r}(E) \rightarrow \mathrm{H}^{r}(E / \mathcal{O}) \rightarrow \cdots,
$$

is concentrated at the three terms of degree $r=d$. From this we deduce the freeness.

\subsection{On the Gorenstein property of the Hecke algebra}

THEOREM 6.7 (Theorem B). - Let $f$ and $p$ be such that $(\mathbf{I}),(\mathbf{I I})$ and $\left(\mathbf{L I}_{\text {Ind } \bar{\rho}}\right)$ hold. Then

(i) $\mathrm{H}^{\bullet}\left(Y, \mathbb{V}_{n}(\kappa)\right)[\mathfrak{m}]=\mathrm{H}^{d}\left(Y, \mathbb{V}_{n}(\kappa)\right)[\mathfrak{m}]$ is a $\kappa$-vector space of dimension $2^{d}$.

(ii) $\mathrm{H}^{\bullet}\left(Y, \mathbb{V}_{n}(\mathcal{O})\right)_{\mathfrak{m}}=\mathrm{H}^{d}\left(Y, \mathbb{V}_{n}(\mathcal{O})\right)_{\mathfrak{m}}$ is free of rank $2^{d}$ over $\mathbb{T}_{\mathfrak{m}}$.

(iii) $\mathbb{T}_{\mathfrak{m}}$ is Gorenstein.

Proof. - In this proof we put $W=\mathrm{H}^{d}\left(Y_{\overline{\mathbb{Q}}}, \mathbb{V}_{n}(\kappa)\right)_{\mathfrak{m}}$. By using an auxiliary level structure as in [8], we can assume that the condition (NT) of Section 1.4 is fulfilled.

(i) As in the proof of Theorem 6.6(i), by Lemma 6.5 we have an isomorphism of $\mathcal{G}_{\widehat{F}^{\prime}}$,-modules

$$
W[\mathfrak{m}]^{\mathrm{s.s.}}=\left(\bigotimes \operatorname{Ind}_{F}^{\mathbb{Q}} \bar{\rho}\right)^{\oplus r}
$$

It is crucial to observe that $I_{p} \subset \mathcal{G}_{\widehat{F}^{\prime}}$. By Theorem 2.6 we have $r \geqslant 1$. In order to show that $r=1$ we consider the restriction of these representations to $I_{p}$. The multiplicity of the maximal Fontaine-Laffaille weight $\left|p\left(J_{F}\right)\right|$ in the right-hand side is $r$ by Theorem 2.6, Corollary 2.7(ii) and Fontaine-Laffaille's theory.

On the other hand, the multiplicity of $\left|p\left(J_{F}\right)\right|$ in the left-hand side is equal, by Theorem 5.13, to the dimension of $\mathrm{H}^{0}\left(\bar{Y} \otimes \kappa, \overline{\mathcal{W}}_{\epsilon_{J_{F}}(n+t)-t, n_{0}}\right)[\mathfrak{m}]$ : In fact, by Remark 5.14 all we have to check is the $T_{\mathfrak{p}}$-linearity of the Fontaine-Laffaille functor gr ${ }^{\left|p\left(J_{F}\right)\right|}$ on $\mathrm{H}^{d}\left(Y_{\overline{\mathbb{Q}}}, \mathbb{V}_{n}(\kappa)\right)_{\mathfrak{m}^{\prime}}$. By Theorem 6.6(ii) the module $\mathrm{H}^{d}\left(Y_{\overline{\mathbb{Q}}}, \mathbb{V}_{n}(\mathcal{O})\right)_{\mathfrak{m}^{\prime}}$ is torsion free. Therefore $T_{\mathfrak{p}}$-linearity may be checked after extending the scalars to $\mathbb{C}$, where it follows from the Strong Multiplicity One Theorem (since $p$ is prime to the level $\mathfrak{n}$ ). We owe this idea to Diamond (see [8, Proposition 1]).

We will now see that $\operatorname{dim}_{\kappa} \mathrm{H}^{0}\left(\bar{Y} \otimes \kappa, \overline{\mathcal{W}}_{\epsilon_{J_{F}}(n+t)-t, n_{0}}\right)[\mathfrak{m}]=1$. We have $\overline{\mathcal{W}}_{\epsilon_{J_{F}}(n+t)-t, n_{0}}=$ $\underline{\omega}^{k} \otimes \underline{\nu}^{n_{0} t / 2}$. So we are led to show that two normalized Hilbert modular forms of weight $k$, level $\mathfrak{n}$ and coefficients in $\kappa=\mathbb{T}_{\mathfrak{m}} / \mathfrak{m}$ having the same eigenvalues for all the Hecke operators are equal. One should be careful to observe that the Hecke operators permute the connected components $M_{1}(\mathfrak{c}, \mathfrak{n})$ of the Shimura variety $Y=Y_{1}(\mathfrak{n})$ (here the ideal $\mathfrak{c}$ runs over a set of representatives of $\mathrm{Cl}_{F}^{+}$). We use Hecke relations between Fourier coefficients and eigenvalues for 
the Hecke operators and the $q$-expansion Principle at the $\infty$ cusp of each connected component $M_{1}(\mathfrak{c}, \mathfrak{n})$ (see Section 1.7).

(ii), (iii) Mazur's argument in the elliptic modular case remains valid. By Theorem A, the twisted Poincaré pairing (6) on $\mathrm{H}^{d}\left(Y, \mathbb{V}_{n}(\mathcal{O})\right)_{\mathfrak{m}}=\mathrm{H}_{c}^{d}\left(Y, \mathbb{V}_{n}(\mathcal{O})\right)_{\mathfrak{m}}$ is a perfect duality of $\mathbb{T}_{\mathfrak{m}}$-modules, so it would be enough to show (ii).

Again using the perfectness of the twisted Poincaré pairing $W \times W \rightarrow \kappa$ we obtain $W \cong$ $\operatorname{Hom}_{\mathbb{T}_{\mathfrak{m}}}(W, \kappa)$, and so $W \otimes_{\mathbb{T}_{\mathfrak{m}}} \kappa=W / \mathfrak{m} W \cong \operatorname{Hom}(W[\mathfrak{m}], \kappa)$, and therefore

$$
\operatorname{dim}_{\kappa}\left(W \otimes_{\mathbb{T}_{\mathfrak{m}}} k\right)=\operatorname{dim}_{\kappa}(W[\mathfrak{m}]),
$$

which equals $2^{d}$, by (i). Then (ii) follows from the following:

LEMmA 6.8. - Let $\mathcal{T}$ be a torsion free local $\mathcal{O}$-algebra $\left(\mathcal{T} \hookrightarrow \mathcal{T} \otimes_{\mathcal{O}}\right.$ E) of maximal ideal $\mathfrak{m}$ and residue field $\kappa=\mathcal{T} / \mathrm{m}$.

Let $\mathcal{M}$ be a finitely generated $\mathcal{T}$-module such that $\mathcal{M} \otimes_{\mathcal{O}} E$ is free of rank $r$ over $\mathcal{T} \otimes_{\mathcal{O}} E$. If $\mathcal{M} \otimes_{\mathcal{T}} \kappa$ is a $\kappa$-vector space of dimension $\leqslant r$, then $\mathcal{M}$ is free of rank $r$ over $\mathcal{T}$.

Proof. - Since $\mathcal{M} \otimes_{\mathcal{T}} k$ is of dimension $\leqslant r$, the Nakayama's lemma gives a surjective homomorphism of $\mathcal{T}$-modules $\mathcal{T}^{r} \rightarrow \mathcal{M}$. Denote by $I$ its kernel. We have an exact sequence of $\mathcal{O}$-modules

$$
0 \rightarrow I \rightarrow \mathcal{T}^{r} \rightarrow \mathcal{M} \rightarrow 0
$$

By tensoring it by $\otimes_{\mathcal{O}} E$ (or equivalently by $\otimes_{\mathcal{T}}\left(\mathcal{T} \otimes_{\mathcal{O}} E\right)$ ) we obtain another exact sequence

$$
0 \rightarrow I \otimes_{\mathcal{O}} E \rightarrow\left(\mathcal{T} \otimes_{\mathcal{O}} E\right)^{r} \rightarrow \mathcal{M} \otimes_{\mathcal{O}} E \rightarrow 0
$$

By comparing the dimensions over $E$ we get $I \otimes_{\mathcal{O}} E=0$. Since $I$ is torsion free, $I=0$.

\subsection{An application to $p$-adic ordinary families}

For $r \geqslant 1$, consider the following open compact subgroups of $G\left(\mathbb{A}_{f}\right)$

$$
\begin{gathered}
K_{0}\left(p^{r}\right)=\left\{u \in K_{1}(\mathfrak{n}) \mid u \equiv\left(\begin{array}{cc}
* & * \\
0 & *
\end{array}\right)\left(\bmod p^{r}\right)\right\} \\
K_{11}\left(p^{r}\right)=\left\{u \in K_{1}(\mathfrak{n}) \mid u \equiv\left(\begin{array}{ll}
1 & * \\
0 & 1
\end{array}\right)\left(\bmod p^{r}\right)\right\} .
\end{gathered}
$$

Let $Y_{0}\left(p^{r}\right)$ (respectively $Y_{11}\left(p^{r}\right)$ ) be the Hilbert modular variety of level $K_{0}\left(p^{r}\right)$ (respectively $K_{11}\left(p^{r}\right)$ ).

The cohomology group $\mathrm{H}^{\bullet}\left(Y_{11}\left(p^{r}\right), \mathbb{V}_{n}(E / \mathcal{O})\right)^{*}$ has a natural action of $K_{0}\left(p^{r}\right) / K_{11}\left(p^{r}\right) \simeq$ $\left(\mathfrak{o} / p^{r}\right)^{\times} \times\left(\mathfrak{o} / p^{r}\right)^{\times}$(we denote by ${ }^{*}$ the Pontryagin dual). Therefore the group $T\left(\mathbb{Z}_{p}\right) / \mathfrak{o}^{\times}$acts on the inductive limit $\mathrm{H}^{\bullet}\left(Y_{11}\left(p^{\infty}\right), \mathbb{V}_{n}(E / \mathcal{O})\right)^{*}:=\lim \mathrm{H}^{\bullet}\left(Y_{11}\left(p^{r}\right), \mathbb{V}_{n}(E / \mathcal{O})\right)^{*}$.

By Hida's stabilization lemma, the ordinary part of $\mathrm{H}^{\bullet}\left(Y_{11}\left(p^{\infty}\right), \mathbb{V}_{n}(E / \mathcal{O})\right)^{*}$ (that is the part where the Hecke operators $T_{0, \mathfrak{p}}$ of Definition 1.13 are invertible for all $\mathfrak{p}$ dividing $p$ ) is independent on $n$. We denote it by $\mathcal{H}_{\text {ord }}^{\bullet}:=\mathrm{H}_{\text {ord }}^{\bullet}\left(Y_{11}\left(p^{\infty}\right), E / \mathcal{O}\right)^{*}$.

By the above discussion $\mathcal{H}_{\text {ord }}^{\bullet}$ has a structure of a $\Lambda:=\mathcal{O}\left[\left[T\left(\mathbb{Z}_{p}\right) / \overline{\mathfrak{o}^{\times}}\right]\right]$-module. It is of finite type, by a theorem of Hida.

We also define the $p$-adic ordinary Hecke $\Lambda$-algebra $\mathbb{T}_{k, \text { ord }}^{\infty}:=\lim _{\leftarrow} \mathbb{T}_{k, \text { ord }}\left(Y_{11}\left(p^{r}\right)\right)$. As $\mathbb{T}_{k \text {,ord }}^{\infty}$ is independent of $k$, we denote it by $\mathbb{T}_{\text {ord }}^{\infty}$. Then $\mathcal{H}_{\text {ord }}^{\bullet}$ is a $\mathbb{T}_{\text {ord }}^{\infty}$-module. 
An arithmetic character of $T\left(\mathbb{Z}_{p}\right) / \overline{\mathfrak{o}^{\times}}$is by definition a character whose restriction to an open subgroup is given by an algebraic character. It is immediate that such a character is a product of an algebraic character and a finite order character. An algebraic character of $T\left(\mathbb{Z}_{p}\right) \simeq D\left(\mathbb{Z}_{p}\right) \times D\left(\mathbb{Z}_{p}\right)$ trivial on $\mathfrak{o}^{\times}$is necessarily of the form $(u, \epsilon) \mapsto u^{n} \epsilon^{-m}$, where $m, n \in \mathbb{Z}\left[J_{F}\right]$ and $m+2 n \in \mathbb{Z} t$. Hence, the general form of an arithmetic character $\psi$ of $T\left(\mathbb{Z}_{p}\right) / \mathcal{o}^{\times}$is $(u, \epsilon) \mapsto u^{n} \epsilon^{-m} \psi_{1}(u) \psi_{2}(\epsilon)$, where $\psi_{1}, \psi_{2}$ are finite order characters. Every such $\psi$ induces an $\mathcal{O}$-algebra homomorphism $\Lambda \rightarrow \mathcal{O}$, whose kernel is denoted by $P_{\psi}$.

Let $\mathfrak{m}$ be a maximal ordinary ideal of $\mathbb{T}$ and let $\mathfrak{m}_{\infty}$ be a maximal ideal of $\mathbb{T}_{\text {ord }}^{\infty}$ above $\mathfrak{m}$. We denote by $\mathbb{T}_{\mathfrak{m}_{\infty}}^{\infty}$ (respectively $\mathcal{H}_{\mathfrak{m}_{\infty}}^{\bullet}$ ) the localization of $\mathbb{T}_{\text {ord }}^{\infty}$ (respectively of $\mathcal{H}_{\text {ord }}^{\bullet}$ ) at $\mathfrak{m}_{\infty}$.

Proposition 6.9. - Let $\mathfrak{m}$ be such that $(\mathbf{I}),(\mathbf{I I})$ and $\left(\mathbf{L I}_{\text {Ind } \bar{\rho}}\right)$ hold. Then

(i) $\mathcal{H}_{\mathfrak{m}_{\infty}}^{d}$ is free of finite rank over $\Lambda$ and we have an exact control:

$$
\mathcal{H}_{\mathfrak{m}_{\infty}}^{d} / P_{\psi} \mathcal{H}_{\mathfrak{m}_{\infty}}^{d} \simeq \mathrm{H}^{\bullet}\left(Y_{11}\left(p^{r}\right), \mathbb{V}_{\psi}(E / \mathcal{O})\right)_{\mathfrak{m}_{r}}^{*}
$$

(ii) $\mathcal{H}_{\mathfrak{m}_{\infty}}^{d}$ is free of rank $2^{d}$ over $\mathbb{T}_{\mathfrak{m}_{\infty}}^{\infty}$, and

(iii) Hida's control theorem for the Hecke algebra holds, that is $\mathbb{T}_{\mathfrak{m}_{\infty}}^{\infty}$ is a free $\Lambda$-algebra of finite rank and for every $\psi$ we have $\mathbb{T}_{\mathfrak{m}_{\infty}}^{\infty} / P_{\psi} \mathbb{T}_{\mathfrak{m}_{\infty}}^{\infty} \simeq \mathbb{T}_{\psi}\left(Y_{11}\left(p^{r}\right)\right)_{\mathfrak{m}_{r}}$.

Proof. - (i) The proof is very similar to the one of [31, Theorem 9]. It uses that a $\Lambda$-module is free, if it is free of constant rank over $\mathcal{O}$ for infinitely many specializations. In our case, it is enough to specialize at weights of the form $k+(p-1) k^{\prime}$ and verify the exact control criterion using Theorem 6.6. We omit the details, because (i) follows from (ii) and (iii).

(ii) Consider $\Lambda \rightarrow \mathbb{T}_{\mathfrak{m}_{\infty}}^{\infty} \rightarrow \operatorname{End}_{\mathcal{O}}\left(\mathcal{H}_{\mathfrak{m}_{\infty}}^{d}\right)$. The specialization at $\psi=\psi_{k}$ gives

$$
\mathcal{O} \rightarrow \mathbb{T}_{\mathfrak{m}_{\infty}}^{\infty} / P_{k} \mathbb{T}_{\mathfrak{m}_{\infty}}^{\infty} \rightarrow \operatorname{End}_{\mathcal{O}}\left(\mathcal{H}_{\mathfrak{m}_{\infty}}^{d} / P_{k} \mathcal{H}_{\mathfrak{m}_{\infty}}^{d}\right)
$$

By Theorem 6.6 we have $\mathrm{H}^{d}\left(Y_{0}(p), \mathbb{V}_{n}(E / \mathcal{O})\right)_{\mathfrak{m}}^{*} \simeq \mathrm{H}^{d}\left(Y, \mathbb{V}_{n}(E / \mathcal{O})\right)_{\mathfrak{m}}^{*} \simeq \mathrm{H}^{d}\left(Y, \mathbb{V}_{n}(\mathcal{O})\right)_{\mathfrak{m}}$ and an exact control: $\mathcal{H}_{\mathfrak{m}_{\infty}}^{d} / P_{k} \mathcal{H}_{\mathfrak{m}_{\infty}}^{d} \simeq \mathrm{H}^{d}\left(Y, \mathbb{V}_{n}(\mathcal{O})\right)_{\mathfrak{m}}$.

From here and from Theorem B we obtain that $\mathcal{H}_{\mathfrak{m}_{\infty}}^{d} \otimes_{\mathbb{T}_{\mathfrak{m}_{\infty}}^{\infty}}\left(\mathbb{T}_{\mathfrak{m}_{\infty}}^{\infty} / P_{\psi} \mathbb{T}_{\mathfrak{m}_{\infty}}^{\infty}\right) \simeq \mathcal{H}_{\mathfrak{m}_{\infty}}^{d} \otimes_{\Lambda}$ $\Lambda / P_{k}$ is free of rank $2^{d}$ over $\mathbb{T}_{\mathfrak{m}}$. Hence $\mathcal{H}_{\mathfrak{m}_{\infty}}^{d} \otimes_{\mathbb{T}_{\mathfrak{m} \infty}^{\infty}} \kappa$ is free of rank $2^{d}$ over $\mathbb{T}_{\mathfrak{m}} \otimes_{\mathbb{T}_{\mathfrak{m} \infty}^{\infty} / P_{\psi} \mathbb{T}_{\mathfrak{m} \infty}^{\infty}}$ $\kappa=\kappa$. Then Lemma 6.8 applies to the $\mathbb{T}_{\mathfrak{m}_{\infty}}^{\infty}$-module $\mathcal{H}_{\mathfrak{m}_{\infty}}^{d}$ which is finitely generated over the local algebra $\Lambda$.

(iii) Since $\mathcal{H}_{\mathfrak{m}_{\infty}}^{d}$ is a free $\Lambda$-module, it admits a direct sum decomposition with respect to the Weyl group action on the Betti cohomology:

$$
\mathcal{H}_{\mathfrak{m}_{\infty}}^{d}=\bigoplus_{J \subset J_{F}} \mathcal{H}_{\mathfrak{m}_{\infty}}^{d}\left[\widehat{\epsilon_{J}}\right]
$$

Every $\mathcal{H}_{\mathfrak{m}_{\infty}}^{d}\left[\widehat{\epsilon_{J}}\right]$ is free of rank 1 over $\mathbb{T}_{\mathfrak{m}_{\infty}}^{\infty}$ and free over $\Lambda$. Therefore $\mathbb{T}_{\mathfrak{m}_{\infty}}^{\infty}$ is free over $\Lambda$ and exact control holds.

COROLlaRY 6.10. - Let $f \in S_{k+(p-1) k^{\prime}}\left(Y_{0}\left(p^{r}\right)\right)$ be a newform and let $p$ be a prime not dividing $\mathrm{N}_{F / \mathbb{Q}}(\mathfrak{d})$, such that $p-1>\sum\left(k_{\tau}-1\right)$ and $\left(\mathbf{L I}_{\text {Ind } \bar{\rho}}\right)$ holds. Then Theorems $\mathrm{A}$ and $\mathrm{B}$ hold.

$4^{\mathrm{e}}$ SÉRIE - TOME $38-2005-\mathrm{N}^{\circ} 4$ 


\section{List of symbols}

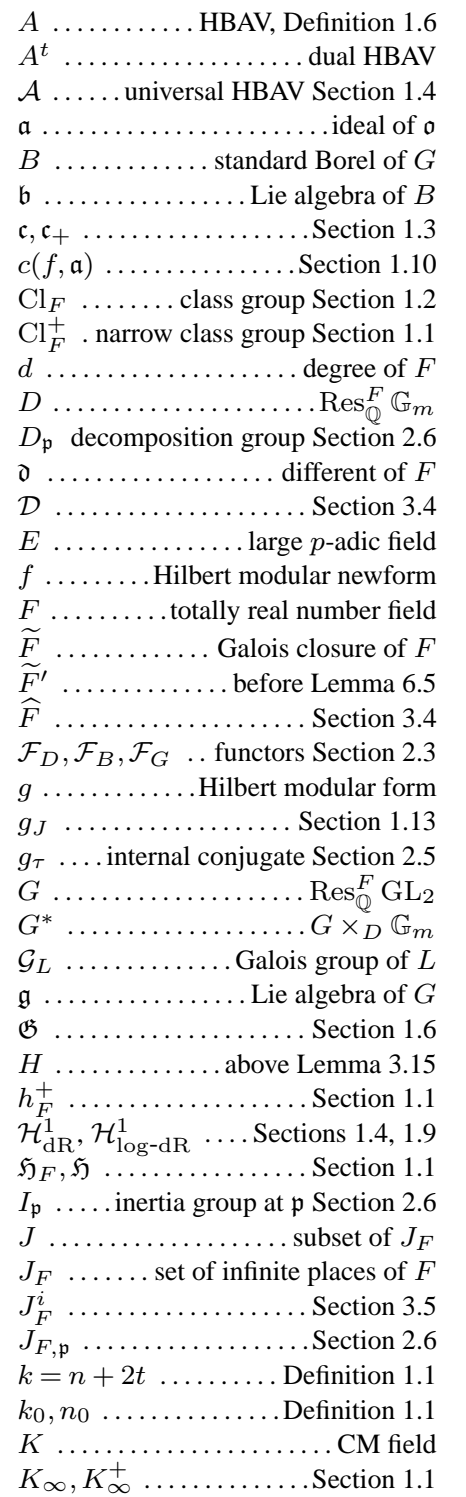

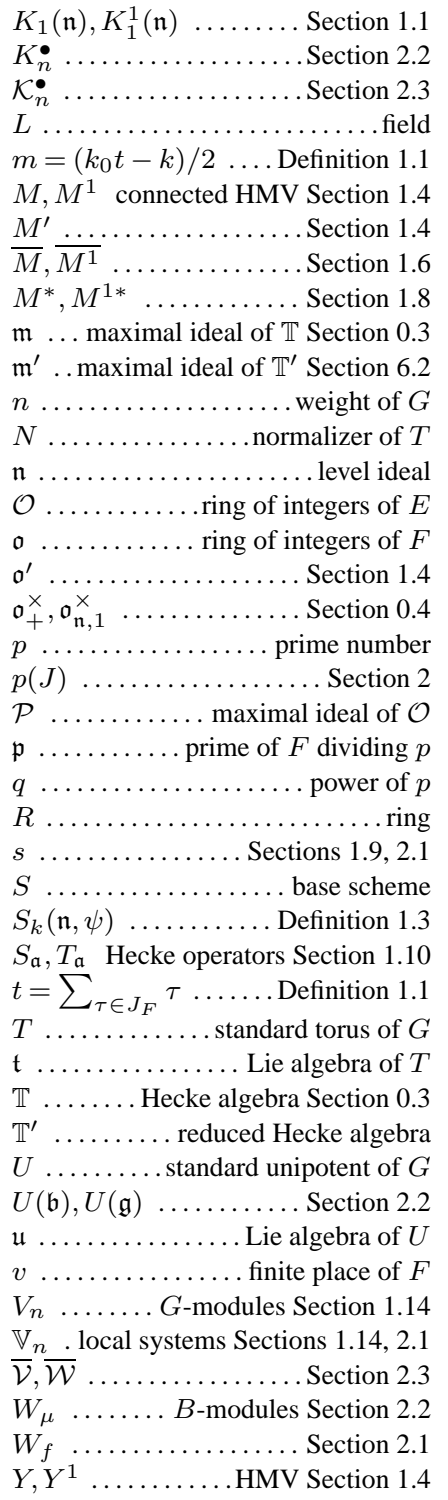

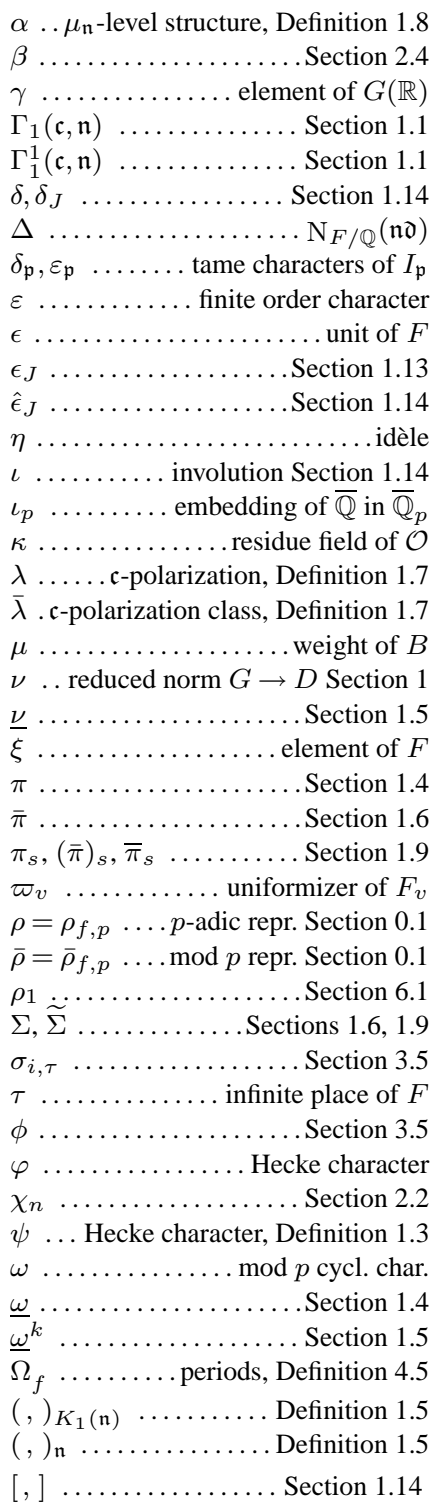

\section{Acknowledgements}

I would like to thank J. Bellaïche, D. Blasius, G. Chenevier, L. Dieulefait, E. Ghate, M. Kisin, V. Lafforgue, A. Mokrane, E. Urban, J. Wildeshaus and J.-P. Wintenberger for helpful conversations. This article was completed during my visit at UCLA on an invitation by H. Hida. I would like to thank him heartily for his hospitality and for many inspiring discussions. I am grateful to G. Kings and R. Taylor for their interesting comments on an earlier version of this paper that have much improved it. Finally, I would like to thank J. Tilouine who suggested that I study this problem and supported me during the preparation of this article. 


\section{REFERENCES}

[1] Blasius D., Rogawski J., Motives for Hilbert modular forms, Invent. Math. 114 (1993) 55-87.

[2] BREuIL C., Une remarque sur les représentations locales $p$-adiques et les congruences entre formes modulaires de Hilbert, Bull. Soc. Math. France 127 (1999) 459-472.

[3] BRYlinski J.-L., LABESSE J.-P., Cohomologie d'intersection et fonctions $L$ de certaines variétés de Shimura, Ann. Sci. École Norm. Sup. 17 (1984) 361-412.

[4] Deligne P., Formes modulaires et représentations $l$-adiques, in: Séminaire Bourbaki, 355, in: Lecture Notes in Math., vol. 179, Springer, Berlin, 1971.

[5] Deligne P., La conjecture de Weil. I, Publ. Math. IHÉS 43 (1974) 273-307.

[6] Deligne P., Valeurs de fonctions $L$ et périodes d'intégrales, in: Proceedings of Symposia of Pure Mathematics, vol. 33, American Mathematical Society, Providence, RI, 1979, pp. 313-346.

[7] Deligne P., Serre J.-P., Formes modulaires de poids 1, Ann. Sci. École Norm. Sup. 7 (1974) $507-$ 530.

[8] Diamond F., On the Hecke action on the cohomology of Hilbert-Blumenthal surfaces, Contemp. Math. 210 (1998) 71-83.

[9] Diamond F., Flach M., Guo L., The Tamagawa number conjecture of adjoint motives of modular forms, Ann. Sci. École Norm. Sup. 37 (2004) 663-727.

[10] Dimitrov M., Compactifications arithmétiques des variétés de Hilbert et formes modulaires de Hilbert pour $\Gamma_{1}(\mathfrak{c}, \mathfrak{n})$, in: Adolphson A., Baldassarri F., Berthelot P., Katz N., Loeser F. (Eds.), Geometric Aspects of Dwork Theory, Walter de Gruyter, Berlin, 2004, pp. 527-554.

[11] Dimitrov M., Tilouine J., Variétés et formes modulaires de Hilbert arithmétiques pour $\Gamma_{1}(\mathfrak{c}, \mathfrak{n})$, in: Adolphson A., Baldassarri F., Berthelot P., Katz N., Loeser F. (Eds.), Geometric Aspects of Dwork Theory, Walter de Gruyter, Berlin, 2004, pp. 555-614.

[12] Dimitrov M., On Ihara's lemma for Hilbert modular varieties, math.NT/0503134.

[13] Faltings G., On the cohomology of locally symmetric Hermitian spaces, in: Séminaire d'algèbre, in: Lecture Notes in Math., vol. 1029, Springer, Berlin, 1983, pp. 349-366.

[14] Faltings G., Crystalline cohomology and p-adic Galois representations, in: Press J.H.U. (Ed.), Algebraic Analysis, Geometry, and Number Theory, 1989, pp. 25-80.

[15] Faltings G., Chai C.-L., Degeneration of Abelian Varieties, Springer, Berlin, 1990.

[16] Faltings G., Jordan B., Crystalline cohomology and GL(2, QQ), Israel J. Math. 90 (1995) 1-66.

[17] Fontaine J.-M., Laffaille G., Construction de représentations p-adiques, Ann. Sci. École Norm. Sup. 15 (1982) 547-608.

[18] GHATE E., Adjoint $L$-values and primes of congruence for Hilbert modular forms, Compositio Math. 132 (2002) 243-281.

[19] HARDER G., Eisenstein cohomology of arithmetic groups. The case $\mathrm{GL}_{2}$, Invent. Math. 89 (1987) 37-118.

[20] HidA H., p-adic Automorphic Forms on Shimura Varieties, Springer, Berlin, 2004.

[21] HidA H., Congruences of cusp forms and special values of their zeta functions, Invent. Math. 63 (1981) 225-261.

[22] HiDA H., On congruence divisors of cuspforms as factors of the special values of their zeta functions, Invent. Math. 64 (1981) 221-262.

[23] HidA H., Nearly ordinary Hecke algebras and Galois representations of several variables, in: Algebraic Analysis, Geometry and Number Theory, Proceedings of the JAMI Inaugural Conference, 1988, pp. $115-134$.

[24] HidA H., On p-adic Hecke algebras for GL 2 over totally real fields, Ann. of Math. 128 (1988) 295384.

[25] HIDA H., On the critical values of $L$-functions of $\mathrm{GL}_{2}$ and $\mathrm{GL}_{2} \times \mathrm{GL}_{2}$, Duke Math. J. 74 (1994) 431-529.

[26] Hida H., Tilouine J., Anti-cyclotomic Katz $p$-adic $L$-functions and congruence modules, Ann. Sci. École Norm. Sup. 26 (1993) 189-259.

[27] ILluSIE L., Réduction semi-stable et décomposition de complexes de de Rham à coefficients, Duke Math. J. 60 (1990) 139-185.

$4^{e}$ SÉRIE - TOME $38-2005-\mathrm{N}^{\circ} 4$ 
[28] JANTZEn J., Representations of Algebraic Groups, Academic Press, New York, 1987.

[29] M. KISIN, K. LAI, Overconvergent Hilbert modular forms, Amer. J. Math., submitted for publication.

[30] Mazur B., Modular curves and the Eisenstein ideal, Inst. Hautes Études Sci. Publ. Math. 47 (1977) 33-186.

[31] Mokrane A., Tilouine J., Cohomology of Siegel varieties with $p$-adic integral coefficients and applications, in: Cohomology of Siegel Varieties, in: Astérisque, vol. 280, 2002, pp. 1-95.

[32] PINK R., On $l$-adic sheaves on Shimura varieties and their higher images in the Baily-Borel compactification, Math. Ann. 292 (1992) 197-240.

[33] Polo P., Tilouine J., Bernstein-Gelfand-Gelfand complexes and cohomology of nilpotent groups over $\mathbb{Z}_{(p)}$ for representations with $p$-small weights, in: Cohomology of Siegel Varieties, in: Astérisque, vol. 280, 2002, pp. 97-135.

[34] Rapoport M., Compactification de l'espace de modules de Hilbert-Blumenthal, Compositio Math. 36 (1978) 255-335.

[35] Ribet K., On l-adic representations attached to modular forms, Invent. Math. 28 (1975) 245-275.

[36] Ribet K., Mod $p$ Hecke operators and congruences between modular forms, Invent. Math. 71 (1983) 193-205.

[37] SERre J.-P., Propriétés galoisiennes des points d'ordre fini des courbes elliptiques, Invent. Math. 15 (1972) 259-331.

[38] Shimura G., The special values of the zeta functions associated with Hilbert modular forms, Duke Math. J. 45 (1978) 637-679.

[39] Steinberg R., Representations of algebraic groups, Nagoya Math. J. 22 (1963) 33-56.

[40] TAYLOR R., On Galois representations associated to Hilbert modular forms, Invent. Math. 98 (1989) 265-280.

[41] TAYLOR R., On Galois representations associated to Hilbert modular forms II, in: Coates J., Yau S.-T. (Eds.), Elliptic Curves, Modular Forms and Fermat's Last Theorem, Hong Kong, 1993, International Press, 1995, pp. 185-191.

[42] Wedhorn T., Congruence relations on some Shimura varieties, J. reine angew. Math. 524 (2000) 43-71.

[43] WiLES A., On ordinary $\lambda$-adic representations associated to modular forms, Invent. Math. 94 (1988) 529-573.

[44] Yoshida H., On the zeta functions of Shimura varieties and periods of Hilbert modular forms, Duke Math. J. 74 (1994) 121-191.

(Manuscrit reçu le 16 février 2004; accepté, après révision, le 31 mars 2005.)

\footnotetext{
Mladen Dimitrov

Université Paris 7,

UFR de Mathématiques,

Case 7012, 2 place Jussieu,

75251 Paris, France

E-mail: dimitrov@math.jussieu.fr
} 